Article

\title{
Cross-Country Potentials and Advantages in Trade in Fish and Seafood Products in the RCEP Member States
}

\author{
Vasilii Erokhin ${ }^{1}$ (D), Gao Tianming ${ }^{1, *}$ and Anna Ivolga ${ }^{2}(\mathbb{D}$ \\ 1 School of Economics and Management, Harbin Engineering University, Harbin 150001, China; basilic@list.ru \\ 2 Faculty of Social and Cultural Service and Tourism, Stavropol State Agrarian University, \\ 355017 Stavropol, Russia; annya_iv@mail.ru \\ * Correspondence: gaotianming@hrbeu.edu.cn; Tel.: +86-136-6461-9594
}

Citation: Erokhin, V.; Tianming, G.; Ivolga, A. Cross-Country Potentials and Advantages in Trade in Fish and Seafood Products in the RCEP Member States. Sustainability 2021, 13, 3668. https://doi.org/10.3390/ su13073668

Academic Editor: Nesar Ahmed

Received: 6 February 2021

Accepted: 22 March 2021

Published: 25 March 2021

Publisher's Note: MDPI stays neutral with regard to jurisdictional claims in published maps and institutional affiliations.

Copyright: (C) 2021 by the authors. Licensee MDPI, Basel, Switzerland. This article is an open access article distributed under the terms and conditions of the Creative Commons Attribution (CC BY) license (https:// creativecommons.org/licenses/by/ $4.0 /)$.

\begin{abstract}
Fisheries has always played a vital role in supporting livelihoods and ensuring food security and sustainable economic and social development in Southeast Asia. Historically, rural and coastal communities across the region have heavily relied on the fish trade as an indispensable source of income and employment. With the establishment of the Regional Comprehensive Economic Partnership (RCEP) between Association of Southeast Asian Nations (ASEAN) economies and large fish traders like China, Japan, South Korea, Australia, and New Zealand, there is a threat for smaller countries to lose competitive advantages in the regional market. By studying bilateral trade flows between fifteen RCEP members in 2010-2019 and matching indicative untapped trade potentials (ITP method) with revealed comparative (RCA method), relative trade (RTA method), and competitive (Lafay index) advantages across 210 pairs of countries, the authors found substantial misbalances between potential values of country-to-country trade and actual advantages of RCEP economies. To optimize gains from intraregional trade for both smaller and larger RCEP members, this study identified advantageous and disadvantageous trading destinations and product categories for individual countries. The recommendations were then generalized along the four groups of economies based on their level of income, contribution to overall RCEP trade in fish, and the share of fishery products in the national trade turnover. From a practical side, the study adds to the knowledge about the fish trade in Asia by detailing how countries can better utilize individual combinations of advantages. From a methodological side, the approach can be employed widely outside the RCEP to establish a reliable picture of potential gains or losses of a particular country in trade with its counterparts across varied sets of competitive advantages.
\end{abstract}

Keywords: ASEAN; comparative advantage; fish; fishery products; RCEP; seafood; trade

\section{Introduction}

For centuries, fish and fishery products have played a crucial role in establishing food security [1], particularly in poor coastal communities [2,3], and contributed to the economic development of many nations around the world [4,5]. With the huge emergence of an international exchange over the last decades, globalization has converged consumption patterns worldwide [6,7] and inextricably linked fish-abundant coastal areas to fish-deficit distant markets, hence affecting the sustainability of fish supply and global food security [8,9]. According to the most recent estimation of the Food and Agriculture Organization (FAO) [10], from 1976 to 2018, the value of global fish exports increased at an annual rate of $8 \%$ in nominal terms and $4 \%$ in real terms. Fishery products have become one of the world's most heavily traded food commodities [11], with over half of exports to developed countries originating from developing economies of Asia, Latin America, and Africa (compared to 39\% in the 1970s) [12]. The share of developing countries of the total quantity of fish production increased from 39\% in 1976 to $60 \%$ in 2018 , supported by the rapid development of aquaculture and investment in export market facilities and infrastructure, particularly in China and Southeast Asia [12]. 
To a considerable degree, the growth of fish production and trade in Asia has been facilitated by a steady liberalization of global agricultural trade $[13,14]$ and an increase in the number of regional trade agreements $[12,15]$. The reduction of import tariffs on fish products, which has become an issue in the international trade negotiations after the Doha Ministerial Meeting in 2001 [16], has been a major driver of fish trade since then [12]. Most countries that have exhausted their gains within national boundaries use trade measures on fish products to secure new markets [17-19]. The growth of intraregional networks in Asia started in 1967 with the establishment of the Association of Southeast Asian Nations (ASEAN) with Indonesia, Malaysia, Philippines, Singapore, and Thailand as founding members [20]. In 1992, ASEAN countries launched the ASEAN Free Trade Area (AFTA), which membership had further expanded by 1999 to ten countries with the accession of Brunei Darussalam, Vietnam, Lao PDR, Myanmar, and Cambodia. By entering into separate trade agreements with other countries of Asia and Oceania, the ASEAN had established a foundation for launching multilateral trade negotiations in 2012 in order to strengthen economic linkages and enhance trade activities among the parties [21]. In November 2020, the resulting Regional Comprehensive Economic Partnership (RCEP) agreement between the ten ASEAN states, Australia, China, Japan, New Zealand, and South Korea established the world's largest free trade area [22] covering a market of 2.2 billion people with a combined size of $30 \%$ of the world's GDP [23].

RCEP countries now play a crucial role in global fisheries production and exports with over $80 \%$ of the world's farmed seafood output and $85 \%$ of 60 million people around the world directly involved in the seafood sector [10]. The region has emerged as a global fish producer, owing to the growth of aquaculture and the large offshore fishing fleet [24]. The Mekong River Basin (Myanmar, Lao PDR, Thailand, Cambodia, and Vietnam) along with huge freshwater areas available in China allow for the production of several million tons of freshwater fish annually [25-27]. The fishery sector is an important contributor to the economic growth of RECP countries and an indispensable source of export revenue for national budgets, as well as a provider of employment [28] and a source of living [29,30], poverty alleviation [31], and food security [32] in coastal communities [10,33,34].

The relevance of fisheries and aquaculture to food security and sustainable development is highlighted by the targets of the United Nations Sustainable Development Goals [35] - the increase in agricultural productivity of fisheries [36], the regulation of fish harvesting and the fight against illegal, unreported, and unregulated fishing [37] and destructive fishing practices [38], and the prevention of trade restrictions and distortions in world agricultural markets [36]. Simplifying trade procedures and lowering import tariffs aims at making fishery products more competitive in the intraregional market $[39,40]$, but current trade in fish in RCEP countries is characterized by three asymmetries.

First is that while bigger traders (China, Japan, Vietnam, Thailand) diversify their trade with the rest of the world, smaller economies critically depend on intraregional trade. China alone accounts for nearly $15 \%$ of the value of the world's total exports of fish and fishery products [41] with nearly two-thirds of the total trade turnover exported to or imported from outside the RCEP. On the other hand, for Cambodia, Lao PDR and Myanmar, where fisheries represent a critical source of export earnings [42], employment of the poor people, and income generation in coastal areas [24], fish trade is mainly generated within the RCEP (Figure 1).

Second, the intra-RCEP trade follows general globalized income inequality pattern demonstrated in earlier studies by Delgado et al. [43], Bene [28], and Mossler [44] when more developed countries act like net importers of low-value fish and seafood from less developed economies and specialize in exporting high-value processed fishery products. Vietnam, Thailand, Myanmar, and Indonesia enjoy a surplus in the intra-RCEP trade in fish with Japan, Australia, South Korea, and Singapore being the top net importers of fish in the region (Figure 2). 


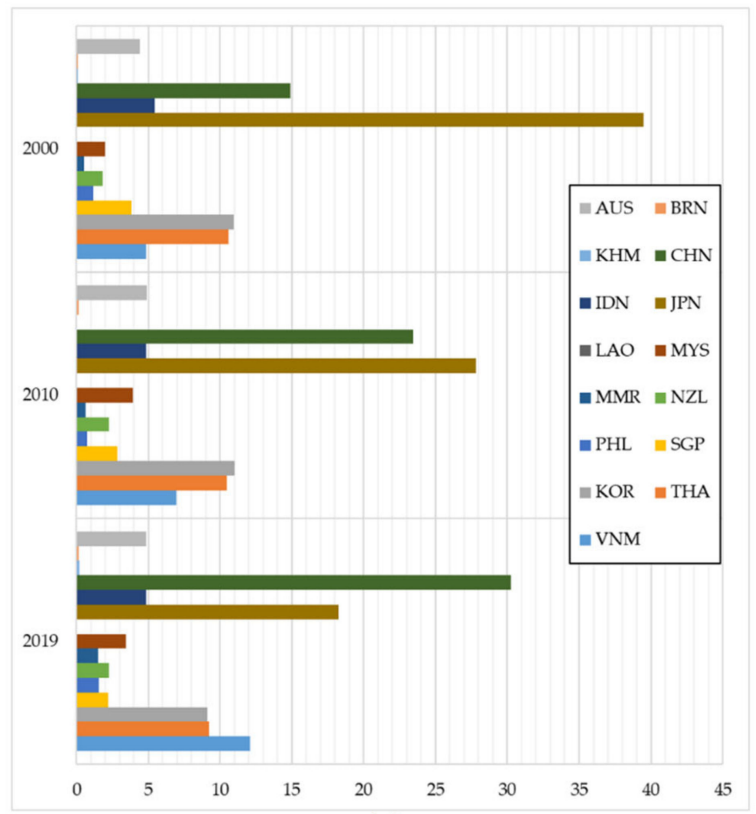

(a)

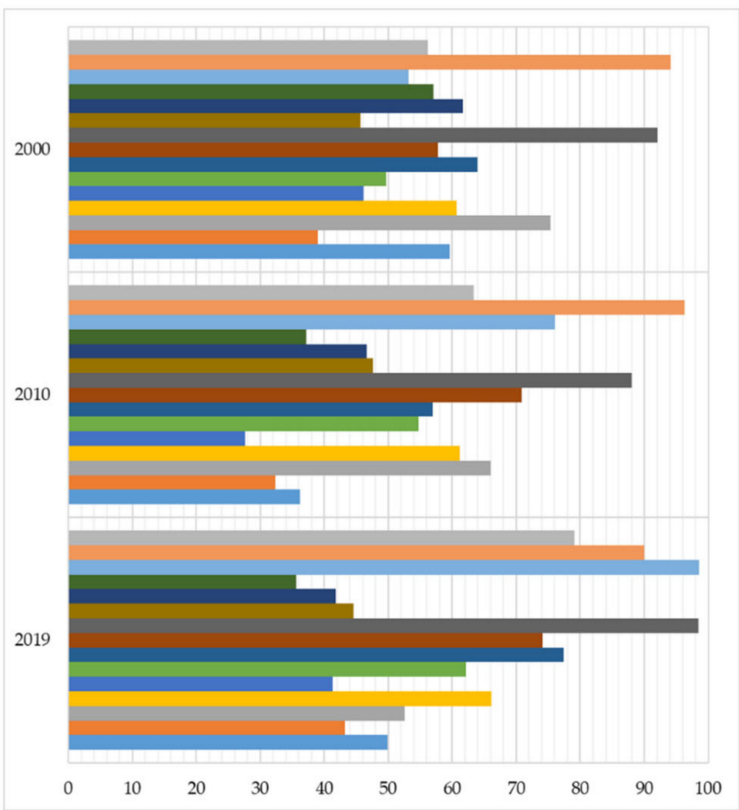

(b)

Figure 1. Regional Comprehensive Economic Partnership (RCEP) trade in fish and seafood products in 2000-2019: (a) share in RCEP trade, (b) RCEP/World trade, \%. Note: country's contribution to the intraregional trade turnover of fish and seafood products; share of the intra-RCEP trade in the country's total trade turnover of fish and seafood products; country abbreviations (AUS = Australia; BRN = Brunei Darussalam; KHM = Cambodia; CHN = China; IDN = Indonesia; JPN = Japan; LAO = Lao PDR; MYS = Malaysia; MMR = Myanmar; NZL = New Zealand; PHL = Philippines; SGP = Singapore; KOR = South Korea; THA = Thailand; VNM = Vietnam). Source: Authors' development based on [45].

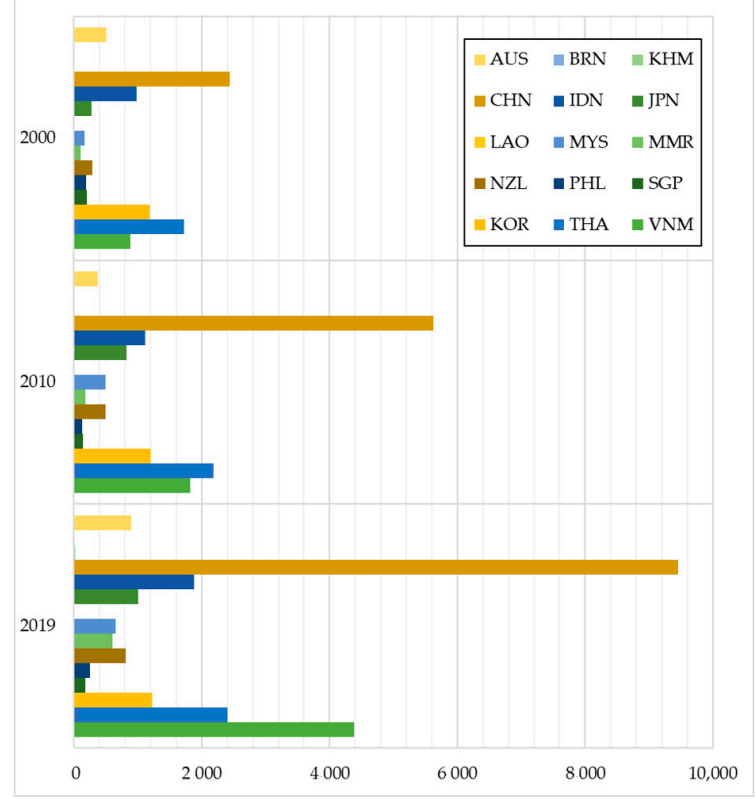

(a)

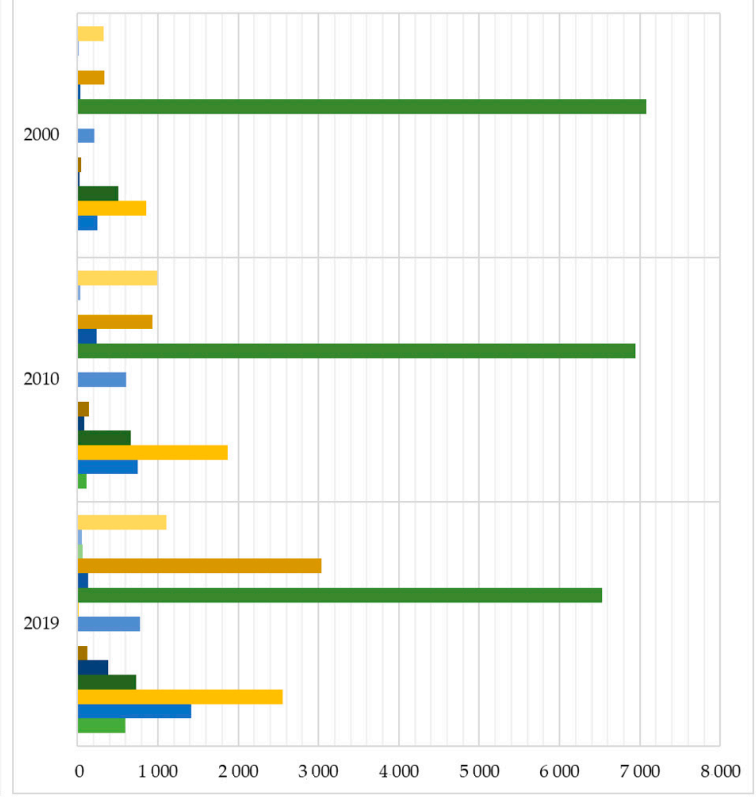

(b)

Figure 2. Intra-RCEP trade in fish and seafood products in 2000-2019: (a) exports to RCEP, (b) imports from RCEP, \$ million. Note: Country abbreviations (AUS = Australia; BRN = Brunei Darussalam; KHM = Cambodia; $\mathrm{CHN}=$ China; IDN = Indonesia; JPN = Japan; LAO = Lao PDR; MYS = Malaysia; MMR = Myanmar; NZL = New Zealand; PHL = Philippines; SGP = Singapore; KOR = South Korea; THA = Thailand; VNM = Vietnam). Source: Authors' development based on [45]. 
The third asymmetry is that the concentration of intraregional trade in fish and seafood products between RCEP members is highly skewed towards higher-income countries, mainly China, Japan, and South Korea, as well as large traders like Vietnam and Thailand, while fishing-dependent lower-income economies make a minor contribution to the intraRCEP trade. Within the RCEP free trade area format, the reductions in import tariffs could further widen this gap by favoring producers in more developed countries at the expense of other exporters, reducing the margins of tariff preferences for less developed economies [46], affecting their competitiveness in the regional market, decreasing their contribution to the intraregional trade, and thus endangering overall stability of economic and social development in smaller RCEP states.

As evidenced by many ASEAN-related and then RCEP-related studies, including Park et al. [47], Prabhakar et al. [48], Yue [49], Erokhin [50], and Menon [51], to achieve stability and improve overall performance, an optimal free trade regime between Asian countries must ensure avoiding misbalances and provide the highest possible gains for all involved parties. From a trade-economic standpoint, the question of optimizing bilateral trade flows is not particularly widely studied. Rather, trade flows in their institutional/regulatory context are frequently estimated with respect to expectations of utility improvements (welfare gains). With this uneven distribution of fish trade in the newly established RCEP, revealing of trade gaps and untapped capacities of individual countries becomes an important knowledge to optimize contributions to and gains from the intraregional trade for a diverse community of higher-income economies (Japan, South Korea, Australia, New Zealand), bigger traders (China, Vietnam, Thailand, Indonesia, Malaysia), smaller countries (Singapore, Brunei Darussalam), less developed economies (Cambodia, Myanmar, Philippines), and even land-locked states (Lao PDR). Capacities and comparative advantages in fish trade of individual countries of Asia and Oceania have been widely investigated, but RCEP-wide studies are very scarce. No study has ever addressed potential trade losses of the RCEP community from the inadequate utilization of advantages of particular countries in the fishery sector. Therefore, in this paper, we aim at revealing potential values of trade in various categories of fish and seafood products between individual RCEP members, studying comparative, trade, and competitive advantages of the fifteen states in country-to-country and country-to region trade, and identifying possible matches between untapped trade potentials and existing advantages in order to optimize gains from the intra-RCEP trade and improve the overall performance of the trade block in the fish trade.

\section{Materials and Methods}

\subsection{Countries, Products, and Data}

The study is performed in the cases of the fifteen RCEP countries (Australia, Brunei Darussalam, Cambodia, China, Indonesia, Japan, Lao PDR, Malaysia, Myanmar, New Zealand, Philippines, Singapore, South Korea, Thailand, and Vietnam) in 2000-2019. According to the SITC Commodity classification of the United Nations Conference on Trade and Development [45], the array of fish and marine food products is established along five $p_{n}$ positions ( $p_{1}=$ fish, crustaceans, mollusks, and preparations thereof, total; $p_{2}=$ fresh, chilled, and frozen fish; $p_{3}=$ dried, salted, and smoked fish; $p_{4}=$ crustaceans, mollusks, and aquatic invertebrates; $p_{5}=$ prepared and preserved fish and aquatic invertebrates). Parameters of trade in $p_{n}$ products (exports; imports, trade turnover, etc.) are calculated individually in 210 pairs of countries [i;j], where a country $i$ exports a product $p_{n}$ to a country $j$ or a combined set of RCEP countries $(r)$ (or imports a product $p_{n}$, respectively).

\subsection{Study Flow Algorithm}

To assess the potentials of individual RCEP countries in the intraregional trade in fish and fishery products, we used the five-stage approach (Table 1). 
Table 1. Study stages.

\begin{tabular}{|c|c|c|}
\hline Stage & Method & Results \\
\hline Trade potential & Indicative Trade Potential (ITP) & $\begin{array}{l}\text { For each pair of } i \text { and } j \text { countries, a } \\
\text { comparison of the current trade turnover } \\
\text { in products } p_{n} \text { with a potential value of } \\
\text { trade. The four-level scale to measure } \\
\text { trade potential. }\end{array}$ \\
\hline Comparative advantage & Revealed Comparative Advantage (RCA) & $\begin{array}{l}\text { Identification of comparative advantages } \\
\text { in trade in products } p_{n} \text { within pairs of } \\
\text { countries } i \text { and } j .\end{array}$ \\
\hline Trade advantage & Relative Trade Advantage (RTA) & $\begin{array}{l}\text { Identification of trade advantages in } \\
\text { trade in products } p_{n} \text { within pairs of } \\
\text { countries } i \text { and } j \text {. Detection of matches } \\
\text { between comparative and } \\
\text { trade advantages. }\end{array}$ \\
\hline Overwriting advantages & Lafay Index (LI) & $\begin{array}{l}\text { Identification of LI values for products } p_{n} \\
\text { traded within pairs of countries } i \text { and } j . \\
\text { Detection of matches between } \\
\text { comparative and trade advantages and } \\
\text { LI values. }\end{array}$ \\
\hline Matching advantages and potentials & Trade potential scale, RCA, RTA, and LI & $\begin{array}{l}\text { The four-level scale to measure } \\
\text { advantages. Detection of countries } i \text { with } \\
\text { high potential and advantages in trade in } \\
\text { products } p_{n} \text { in }[i ; j] \text { and }[i ; r] \text { pairs. }\end{array}$ \\
\hline
\end{tabular}

Source: Authors' development.

At Stage 1, we aggregate parameters of the intraregional trade of the fifteen countries, average the values of exports, imports, and trade turnover (both total trade and $p_{n}$ products) in 2000-2019, calculate indicative trade potentials based on the averaged data, and reveal the degree of fulfillment of trade potential in $[i ; j]$ pairs. At Stages $2-4$, we sequentially calculate the Revealed Comparative Advantage (RCA), the Relative Trade Advantage (RTA), and the Lafay Index (LI) indexes based on averaged data for 2000-2019 for five $p_{n}$ products and 210 pairs of countries and identify countries that demonstrate advantages in the three parameters. Finally, at Stage 5, we match trade potentials with advantages to find intersections and identify the most promising $p_{n}$ products for trade in $[i ; j]$ and $[i ; r]$ pairs. Sections 2.3-2.7 explain the methods and approaches in more detail.

\subsection{Trade Potential}

In light of the existing asymmetries in the RCEP intraregional trade in fish and seafood products (which could probably emerge with the accession of large seafood traders like China, Japan, and South Korea to the agreement), it is crucial to estimate how free trade could help RCEP economies maximize their mutual gains and better utilize their advantages. Therefore, the study starts with examining whether there is an untapped trade potential between particular countries in the block.

A common approach to calculating trade potentials between the two countries or potential value of trade within a free trade area or any other set of countries is to find how far a country-to-country trade deviates from a country-to-region trade. Potential trade can be estimated by matching the total exports of a commodity from one country with the total imports of that commodity to another country [20]. As elaborated by Mukherji [52,53], when comparing exports and imports in such a way, a potential expansion of trade under free trade conditions can be estimated after subtracting existing trade-a technique known as indicative trade potential, or ITP (Equation (1)):

$$
\operatorname{ITP} P_{i j}^{p}=\min \left(X_{i r}^{p} ; M_{j r}^{p}\right)-X_{i j^{\prime}}^{p}
$$


where $I T P_{i j}^{p}=$ indicative trade potential of a country $i$ in trade in a product $p$ with a country $j ; X_{i r}^{p}=$ exports of a product $p$ from a country $i$ to all countries of the region $r ; M_{j r}^{p}=$ imports of a product $p$ to a country $j$ from all countries of the region $r ; X_{i j}^{p}=\operatorname{exports~of~a~product~} p$ from a country $i$ to a country $j$.

The Indicative Trade Potential (ITP) method has been widely used in assessing trade in food and other products both within ASEAN and between ASEAN and the countries of Asia and Oceania [20,54-57]. Compared to estimation trade models and gravity equations [58-60], the use of the ITP method is particularly beneficial in studying bilateral trade flows in the intra-region trade. A gravity model is effective when in addition to the intra-region trade, extra-area countries are considered to capture traded diversion effects and asymmetric shocks coming from other regions. In measuring intra-region trade, gravity model results could be distorted by the distance variable. The ITP approach allows one to identify products for which trade complementarity between the two countries is the highest irrespective of a distance between them or other extra-area parameters [61]. According to Helmers and Pasteels [62], the indicative trade potential in the [i;j] pair of countries shows the degree to which a country $i$ could in principle direct all of its exports of a product $p$ to a country $j$, or, respectively, a degree to which a country $j$ could potentially absorb all imports of a product $p$ from a country $i$. Another rationale for using trade potential as a baseline in our study is that the index has been widely applied in the literature in conjunction with the RCA, the RTA, and other trade-related indicators to test the complementarity or competitiveness in agricultural products between the two trading partners. The successful demonstrations of combined use of trade indexes can be found in Qayyum and Nigar [56], $\mathrm{Li}$ and Li [63], Bano [54], Kapuya et al. [64], Kaur et al. [65], and other studies of bilateral food trade flows in Asia.

Having calculated the ITPs for $[i ; j]$ pairs of RCEP countries, we then compare potentials with actual trade turnover (TT) between countries (average for 2000-2019) by finding the TT/ITP ratio $\left(R_{i j}\right)$. We proceed from the assumption that the higher $R_{i j}$, the closer the ITP to the TT (which means less room for untapped potential, potential trade is close to actual trade $\left(R_{i j}\right.$ tends to 1$)$ or even exceeds it $\left.\left(R_{i j}>1\right)\right)$. Conversely, the closer $R_{i j}$ to 0 the higher the degree of untapped potential of a country $i$ in trade with a country $j$. To be able to differentiate $R_{i j}$ values in terms of the gap between actual and potential trade, we introduce a four-level scale. Simple averaging of all $R_{i j}$ values in the set results in the average value $\left(\overline{R_{i j}}\right)$. The upper and the lower limits of $\overline{R_{i j}}$ are derived by averaging of $R_{i j m a x}$ and $\overline{R_{i j}}$ and $R_{i j m i n}$ and $\overline{R_{i j}}$, respectively. The degree of trade potential across [i;j] pairs of countries is identified by the falling of $R_{i j}$ into one of the categories: high, above average, below average, and low (Table 2).

Table 2. Scale to measure the degree of trade potential.

\begin{tabular}{cc}
\hline Type of Trade Potential & Scale \\
\hline Type 1: high & $\frac{R_{i j \min }+\overline{R_{i j}}}{2}>R_{i j} \geq R_{i j \min }$ \\
Type 2: above average & $\overline{R_{i j}}>R_{i j} \geq \frac{R_{i j m i n}+R_{i j}}{2}$ \\
Type 3: below average & $\frac{R_{i j \max }+\overline{R_{i j}}}{2}>R_{i j} \geq \overline{R_{i j}}$ \\
Type 4: low & $\frac{R_{i j \max }+\overline{R_{i j}}}{2} \leq R_{i j} \leq R_{i j m a x}$ \\
\hline
\end{tabular}

Source: Authors' development.

\subsection{Comparative Advantage}

It is commonly accepted that the value of trade between the countries is affected by their comparative advantages $[66,67]$ which can be measured by the Balassa index of revealed comparative advantage (RCA) [68] (Equation (2)): 


$$
R C A_{i j}^{p}=\frac{\frac{X_{i j}^{p}}{X_{i j}}}{\frac{X_{r}^{p}}{X_{r}}}
$$

where $R C A_{i j}^{p}=$ revealed comparative advantage of a country $i$ in trade in a product $p$ with a country $j ; X_{i j}^{p}=$ exports of a product $p$ from a country $i$ to a country $j ; X_{i j}=$ total exports from a country $i$ to a country $j ; X_{r}^{p}=$ exports of a product $p$ within the region $r ; X_{r}=$ total intraregional exports.

This method has been employed by many scholars to identify comparative advantages in food and agricultural trade (including fish and seafood) within the RCEP [69], the ASEAN [59,70,71], and between ASEAN countries and China [72,73], Japan [74], South Korea [75], and other economies of Asia [76-79]. $R C A_{i j}^{p}>1$ denotes a comparative advantage of a country $i$ in trade in a product $p$ with a country $j$, while $R C A_{i j}^{p}<1$ means a comparative disadvantage, respectively. This means that a country $i$ specializes in the export of a product $p$ to a country $j$ if the market share of this product in trade in $[i ; j]$ pair is above average or if the weight of a product $p$ in the total export of a country $i$ to a county $j$ is higher than the weight of a product $p$ in the aggregated RCEP exports [80].

Despite its wide use in studies of trade flows between countries, in some cases, the utilization of the RCA approach can result in rather rough or even misleading estimations [81]. According to many authors, including Balance et al. [82], Coniglio et al. [83], Tampubolon [73], and Gnidchenko and Salnikov [84], the comparative advantage measure must express the ratio of actual trade and reflect net trade rather than exports only. Moreover, due to its static nature, the RCA does not account for market volatilities in the long run, which limits its application in studying new free trade areas and multilateral trade agreements in their dynamics. Also, as emphasized by Yeats [85] and Hoen and Oosterhaven [86], the application of the RCA to a set of countries significantly different in size (the case of smaller ASEAN countries and large fish traders like China or Japan) can misleadingly result in excessively strong advantages for smaller economies.

\subsection{Relative Trade Advantage}

To avoid possible inaccuracies in measurement, we test revealed comparative advantages by the Vollrath index of relative trade advantage (RTA). So far, such a two-index technique has been rarely used in assessing advantages in fish trade in Asia (except by Khai et al. [87] when measuring comparative advantages of shrimp trade in Malaysia), but it has proven itself in the studies of agricultural trade in Europe [88-90], Latin America [91], and the Middle East [92]. In contrast to the RCA, the RTA accounts for both exports and imports and thus demonstrates net trade advantages (Equation (3)):

$$
R T A_{i j}^{p}=\frac{\frac{X_{i j}^{p}}{X_{i j}}}{\frac{X_{r}^{p}}{X_{r}}}-\frac{\frac{M_{i j}^{p}}{M_{i j}^{p}}}{\frac{M_{r}^{p}}{M_{r}}},
$$

where $R T A_{i j}^{p}=$ relative trade advantage of a country $i$ in trade in a product $p$ with a country $j$; $X_{i j}^{p}=$ exports of a product $p$ from a country $i$ to a country $j ; X_{i j}=$ total exports from a country $i$ to a country $j ; X_{r}^{p}=$ exports of a product $p$ within the region $r ; X_{r}=$ total intraregional exports; $M_{i j}^{p}=$ imports of a product $p$ to a country $i$ from a country $j ; M_{i j}=$ total imports to a country $i$ from a country $j ; M_{r}^{p}=$ imports of a product $p$ within the region $r ; M_{r}=$ total intraregional imports.

$R T A_{i j}^{p}>0$ demonstrates that a country $i$ enjoys a relative advantage in trade in a product $p$ with a country $j$, while $R T A_{i j}^{p}<0$ shows a comparative disadvantage, respectively. 


\subsection{Overwriting Advantages}

Having identified products $p$ in $[i ; j]$ pairs for which $R T A_{i j}^{p}>0$, we then applied those results upon previously calculated RCAs, compared the sets, and revealed the matches between the two advantages. However, since both the RCA and the RTA are structural parameters of advantages [93] and are not always consistent in cardinal and ordinal measures $[82,87,94]$, it is important to eliminate the influence of cyclical factors by using an alternative measure [95]. In cases of ASEAN countries, Karimi and Malekshahian [96] and Oberoi [97] tested revealed advantages by Lafay index (LI) (Equation (4)). This measure allows one to test both comparative and trade advantages by considering the difference between the normalized trade balance of a product and the overall normalized trade balance of a country $[98,99]$.

$$
L I_{i j}^{p}=100 \times\left[\frac{X_{i j}^{p}-M_{i j}^{p}}{X_{i j}^{p}+M_{i j}^{p}}-\frac{X_{i r}^{p}-M_{i r}^{p}}{X_{i r}^{p}+M_{i r}^{p}}\right] \times \frac{X_{i j}^{p}+M_{i j}^{p}}{X_{i r}^{p}+M_{i r}^{p}},
$$

where $L I_{i j}^{p}=$ Lafay index of a country $i$ in trade in a product $p$ with a country $j ; X_{i j}^{p}=$ exports of a product $p$ from a country $i$ to a country $j ; X_{i r}^{p}=\operatorname{exports~of~a~product~} p$ from a country $i$ to the region $r ; M_{i j}^{p}=$ imports of a product $p$ to a country $i$ from a country $j ; M_{i r}^{p}=$ imports of a product $p$ to a country $i$ from the region $r$.

To the best of the authors' knowledge, the LI has never been applied to testing the comparative and trade advantages of RCEP countries in the fishery sector. However, based on several successful evidences of earlier use of the three-indexes approach in various segments of food markets and supply chains in Europe [100-105], Asia [80,95,106,107], and Africa $[108,109]$, we applied the Lafay index for testing the RCA and the RTA datasets across $[i ; j]$ pairs of RCEP countries and $p_{n}$ product categories. If $L I_{i j}^{p}>0$, a country $i$ possesses a competitive advantage in trade in a product $p$ with a country $j$, otherwise, there is a disadvantage. The particular value of the LI is that it captures intraregional trade flows by employing both the exports and imports variables [110]. For the purpose of this study, it is also beneficial that the LI does not take into account world variables [66], which is important in building reliable estimates of trade advantages of individual countries within the RCEP.

\subsection{Matching Advantages and Potentials}

The consecutive calculation of the RCA, the RTA, and the LI indexes for the same array of trade data results in a varied presentation of comparative, trade, and competitive advantages in the fish trade. To measure the degree of advantages of countries $i$ in trade in certain products $p_{n}$ in $[i ; j]$ pairs, we differentiate the values of the three indexes in the following way (Table 3):

Table 3. Scale to measure the degree of advantages.

\begin{tabular}{cc}
\hline Type of Advantage & Criteria \\
\hline Strong advantage (S) & RCA $>1$, RTA $>0$, and LI $>0$ \\
Average advantage (A) & RTA $>0$ and LI $>0$ \\
Weak advantage (W) & RCA $>1$, or RTA $>0$, or LI $>0$ \\
Disadvantage (D) & RCA $<1$, RTA $<0$, and LI $<0$ \\
\hline
\end{tabular}

Source: Authors' development.

The scale is based on previous theoretical insights into establishing the aggregate measure of trade competitiveness on the three-indexes basis (for instance, in Maitah et al. [99], Alessandrini et al. [110], Sanidas and Shin [66], Benesova et al. [101], and Szczepaniak [102]), as well as empirical applications of similar three-indexes measures made by Ishchukova [111], Benesova et al. [100], Smutka et al. [105], Erokhin et al. [80], Erokhin and Gao [95], and Verter et al. [108]. Proceeding from the findings of these studies, 
we assume that when $R C A>1, R T A>0, L I>0$, a country $i$ possesses strong comparative, trade, and competitive advantages ( $S$ type) in trade in a product $p$ with a country $j$. If a country $i$ demonstrates an advantage in both RTA and LI indexes, its advantage is defined as average (A type). In any other case, a country $i$ is recognized as either having a weak advantage (W type) or a disadvantage (D type). Due to previously addressed (Section 2.4) possible inaccuracies in the measurement of comparative advantages, the $[i ; j]$ pairs, where the RCA advantage matches either the RTA or the LI (RCA $>1$ and LI $>0$ or RCA $>1$ and RTA > 0) are not considered as A type. Moreover, as of Dunmore [112], Balance et al. [82], Tampubolon [73], and Leromain and Orefice [81], the RCA index is insufficient for describing the competitive positions of counterparts in bilateral trade, since it allows identifying comparative advantages rather than determining the underlying sources of such advantages. Also, according to Siggel [113], Costinot et al. [114], and Hinloopen and van Marrewijk [115], the RCA index fails to reveal the reasons of advantages and does not allow one to differentiate between natural and acquired advantages (which is crucial for any food production industry, including fisheries). Therefore, a competitive advantage in trade shall be regarded as confirmed only when the RCA is supported by both competitive (LI) and trade (RTA) parameters. To determine whether a country goes to W or D category, we compare matches between indexes. Those countries $i$ for which $R C A<1, R T A<0$, and $L I<0$ are recognized as having a disadvantage (D type). Countries $i$ for which at least one of the indexes demonstrates an advantage are considered as having a weak advantage (W type) in trade in a product $p$ within $[i ; j]$ pairs. Finally, we match the four-level trade advantages scale (Table 3) with the four-level trade potentials scale (Table 2) to detect how untapped trade potentials of individual RCEP countries can be improved by increasing or decreasing trade flows in particular fishery products.

\section{Results}

\subsection{Actual and Potential Trade}

We started with revealing how actual values of trade turnover of $p_{n}$ products within $[i ; j]$ pairs of countries coincide with indicative trade potentials. In general, most of the countries have the potential to increase exports of fish and seafood products to their RCEP counterparts. Among fifteen RCEP member states, only China, Indonesia, Brunei Darussalam, and Lao PDR fulfill their trade potentials in the overall intraregional trade in fish (Appendix A, Table A1). Bilateral trade in fish in the RCEP is asymmetrical. The trade-to-potential ratio demonstrates a significant gap between actual and possible trade in all kinds of fishery products in Myanmar, New Zealand, and Philippines. China, the largest intra-RCEP fish trader, performs rather well in trade with Indonesia, Myanmar, New Zealand, Philippines, and South Korea (actual trade turnover exceeds indicative trade potential), while there is room for expansion of exports from China to Brunei Darussalam, Cambodia, Australia, Lao PDR, and Singapore (high trade potential in [China;j] pairs). Smaller traders like Myanmar, Philippines, New Zealand, and Cambodia experience wider TT/ITP gaps compared with China, Japan, South Korea, and Thailand. This tendency is observed across all four sub-categories of fishery products included in the study. The highest untapped trade potentials are revealed for Cambodia, Myanmar, and Philippines in trade in fresh, chilled, and frozen fish $\left(p_{2}\right)$ (Appendix A, Table A2) and dried, salted, and smoked fish $\left(p_{3}\right)$ (Appendix A, Table A3) and New Zealand and Australia in trade in crustaceans and mollusks $\left(p_{4}\right)$ (Appendix A, Table A4) and prepared and preserved fish and aquatic invertebrates $\left(p_{5}\right)$ (Appendix A, Table A5).

\subsection{Three Types of Advantages}

Having evidenced the asymmetries between larger and smaller traders in both $[i ; j]$ and $[i ; r]$ pairs, we then aim to find out how these differences can be stipulated by comparative advantages of particular $i$ countries in trade in certain $p_{n}$ products. Based on the average RCA values in 2000-2019, the strongest comparative advantages in overall trade in fish and seafood products $\left(p_{1}\right)$ are revealed for Myanmar in its trade with Australia, Brunei 
Darussalam, Malaysia, and Japan, for Vietnam in trade with Japan, South Korea, and Thailand, for New Zealand in trade with Cambodia, Brunei Darussalam, and Japan, and for Thailand in trade with Japan, Australia, and South Korea (Table 4).

Table 4. Comparative, trade, and competitive advantages of RCEP countries in total trade in fish, crustaceans, and mollusks $\left(p_{1}\right)$.

\begin{tabular}{|c|c|c|c|c|c|c|c|c|c|c|c|c|c|c|c|c|c|}
\hline Countries & Indexes & AUS & BRN & КНM & $\mathrm{CHN}$ & IDN & JPN & LAO & MYS & MMR & NZL & PHL & SGP & KOR & THA & VNM & RCEP \\
\hline \multirow[t]{3}{*}{ AUS } & RCA & & - & - & - & - & - & - & - & - & - & - & - & - & - & + & - \\
\hline & RTA & & + & - & - & - & + & + & - & - & - & - & + & - & - & + & - \\
\hline & LI & & - & - & + & - & + & + & - & - & - & - & + & - & - & + & - \\
\hline \multirow{3}{*}{ BRN } & RCA & - & & - & - & - & - & - & - & - & - & - & - & - & - & - & - \\
\hline & RTA & - & & - & - & - & - & + & - & - & - & - & - & - & - & - & - \\
\hline & LI & + & & - & + & + & + & + & - & - & - & - & - & + & + & - & - \\
\hline \multirow[t]{3}{*}{ KHM } & RCA & + & - & & - & - & - & + & - & - & - & - & - & - & + & - & - \\
\hline & RTA & + & + & & + & - & - & + & + & - & - & - & + & + & + & - & - \\
\hline & LI & + & + & & + & - & + & + & + & + & - & - & + & + & - & - & - \\
\hline \multirow{3}{*}{$\mathrm{CHN}$} & RCA & - & - & - & & - & + & - & + & - & - & + & - & + & + & - & + \\
\hline & RTA & + & - & - & & - & + & + & + & - & - & + & + & + & + & - & + \\
\hline & LI & - & - & - & & - & + & + & + & - & - & - & + & + & - & - & + \\
\hline \multirow[t]{3}{*}{ IDN } & RCA & + & - & - & + & & + & - & + & - & - & - & - & - & + & + & + \\
\hline & RTA & + & + & + & + & & + & + & + & - & + & - & + & + & + & + & + \\
\hline & LI & - & + & + & - & & + & + & - & - & - & - & - & + & + & + & + \\
\hline \multirow[t]{3}{*}{ JPN } & RCA & - & - & - & - & - & & - & - & - & - & - & - & - & - & - & - \\
\hline & RTA & - & + & + & - & - & & + & - & - & - & - & - & - & - & - & - \\
\hline & LI & - & + & + & - & - & & + & + & - & + & - & + & + & + & - & - \\
\hline \multirow[t]{3}{*}{ LAO } & RCA & - & - & - & - & - & - & & - & - & - & - & - & - & - & - & - \\
\hline & RTA & - & + & - & - & - & - & & - & + & - & + & - & + & - & - & - \\
\hline & LI & - & + & - & - & - & - & & + & + & - & - & + & + & - & - & - \\
\hline \multirow[t]{3}{*}{ MYS } & RCA & - & + & - & - & - & - & - & & - & - & - & - & - & - & + & - \\
\hline & RTA & + & + & - & - & - & + & + & & - & - & - & + & + & - & - & - \\
\hline & LI & + & + & + & - & - & + & + & & - & - & - & + & + & - & + & - \\
\hline \multirow[t]{3}{*}{ MMR } & RCA & + & + & - & + & + & + & - & + & & + & - & + & + & + & + & + \\
\hline & RTA & + & + & + & + & + & + & + & + & & + & + & + & + & + & + & + \\
\hline & LI & + & + & + & + & - & + & + & + & & - & + & + & + & - & + & + \\
\hline \multirow[t]{3}{*}{ NZL } & RCA & + & + & + & + & - & + & + & + & - & & - & + & + & + & + & + \\
\hline & RTA & + & + & + & + & - & + & + & + & - & & - & + & - & - & - & + \\
\hline & LI & + & + & + & + & - & + & + & - & - & & - & + & - & - & - & + \\
\hline \multirow[t]{3}{*}{ PHL } & RCA & - & + & - & - & - & + & - & - & - & - & & - & - & - & - & - \\
\hline & RTA & + & + & + & - & + & + & + & + & - & + & & + & + & + & - & + \\
\hline & LI & + & + & + & - & - & + & + & + & - & - & & + & + & + & - & + \\
\hline \multirow[t]{3}{*}{ SGP } & RCA & - & - & - & - & - & - & - & - & - & - & - & & - & - & - & - \\
\hline & RTA & - & + & - & - & - & - & + & - & - & - & - & & - & - & - & - \\
\hline & LI & - & + & + & - & - & + & + & + & - & - & - & & + & - & - & - \\
\hline \multirow{3}{*}{ KOR } & RCA & - & - & - & - & - & + & - & - & - & + & - & - & & + & - & - \\
\hline & RTA & + & + & - & - & - & + & - & - & - & + & - & - & & - & - & - \\
\hline & LI & + & + & - & - & - & + & + & - & - & + & - & + & & + & - & - \\
\hline \multirow[t]{3}{*}{ THA } & RCA & + & + & - & - & - & + & - & - & - & + & - & - & + & & + & + \\
\hline & RTA & + & + & - & - & - & + & + & + & - & - & - & + & + & & - & + \\
\hline & LI & + & + & + & - & - & + & + & + & - & - & - & + & - & & - & + \\
\hline \multirow{3}{*}{ VNM } & RCA & + & + & - & + & - & + & - & + & - & + & + & + & + & + & & + \\
\hline & RTA & + & + & + & + & - & + & + & + & - & + & + & + & + & + & & + \\
\hline & LI & + & + & + & - & - & + & + & - & - & - & - & + & + & - & & + \\
\hline
\end{tabular}

Note: Country abbreviations (AUS = Australia; BRN = Brunei Darussalam; KHM = Cambodia; CHN = China; IDN = Indonesia; JPN = Japan; LAO = Lao PDR; MYS = Malaysia; MMR = Myanmar; NZL = New Zealand; PHL = Philippines; SGP = Singapore; KOR = South Korea; THA = Thailand; VNM = Vietnam); "+" = advantage; "-" = disadvantage. Source: Authors' development.

The RCA results show that smaller intraregional traders tend to enjoy higher comparative advantages in trade in $p_{1}$ product category than the largest economies of the block (China, Japan, and South Korea). Comparative advantages in trade in $p_{2-5}$ product categories are significant for Myanmar $\left(p_{2}, p_{3}\right.$, and $\left.p_{4}\right)$, New Zealand $\left(p_{2}, p_{4}\right.$, and $\left.p_{5}\right)$, Vietnam $\left(p_{2}, p_{3}, p_{4}\right.$, and $\left.p_{5}\right)$, and Thailand ( $\left.p 5\right)$ (Appendix B, Tables A6-A10). Thus, we can say that in most $[i ; j]$ pairs, lower $R_{i j}$ values correspond with higher $R C A_{i j}$, that is, larger untapped trade potentials coincide with stronger comparative advantages. However, when this relation is measured in $[i ; r]$ pairs, it is contradictory in Philippines and Cambodia (low $R_{i r}$ and low $R C A_{i r}$ ) and Brunei Darussalam and Lao PDR (high $R_{i r}$ and low $R C A_{i r}$ ). For bigger 
traders, lower values of TT/ITP ratio are generally associated with lower comparative advantages. Exceptions are China, in cases of trade in prepared and preserved fish and aquatic invertebrates $\left(p_{5}\right)$ and fresh, chilled, and frozen fish $\left(p_{2}\right)$, and Indonesia in the cases of trade in dried, salted, and smoked fish $\left(p_{3}\right)$ and crustaceans, mollusks, and aquatic invertebrates $\left(p_{4}\right)$.

The RTA values confirm the relationship between trade potentials and advantages, but with several exceptions. The same six countries (Myanmar, Vietnam, New Zealand, Thailand, Indonesia, and China) enjoy both types of advantages in the overall country-to-region trade in fish and seafood products $\left(p_{1}\right)$ but Thailand's trade advantage is substantially weaker compared to its comparative advantage. Bigger traders, such as China and Indonesia, possess weaker advantages compared to smaller countries (Vietnam, New Zealand, and Myanmar). For the former, the most promising trading destinations are higher-income markets like Japan (high RTAs match with RCAs in $p_{3}$ and $p_{5}$ ) and South Korea ( $p_{2}$ and $\left.p_{4}\right)$. For smaller traders, both comparative and trade advantages are the strongest in the array (all types of fish $\left(p_{2}\right.$ and $\left.p_{3}\right)$ for Myanmar and New Zealand and $p_{2-5}$ for Vietnam).

However, in many $[i ; j]$ pairs, countries trade in $p_{n}$ products in which they possess only relative trade advantages and no comparative ones. Thereby, we applied the Lafay index to test advantages by identifying the difference between the normalized trade balance of a product $p_{n}$ and the overall normalized trade balance of a country $i$ in trade with a country $j$. In most pairs of countries, the LI confirms previously identified relative trade or revealed comparative advantages. In those cases, where the LI does not match the RTA (a criterion for an advantage to be recognized as either average or weak), we distinctly observe two tendencies-depending on whether the LI cancels relative trade advantage or establishes a competitive advantage in the RTA-disadvantageous pairs.

Three types of advantages in the country-to-region overall trade in fish, crustaceans, and mollusks $\left(p_{1}\right)$ are confirmed for the same array of six countries, but the strength of competitive advantages is different from that in the cases of comparative and trade advantages. The highest values of the LI are recorded for Myanmar and Vietnam (first and second ranks in all three indexes, respectively), then follow Thailand (fourth in the RCA and sixth in the RTA), New Zealand (third in the RCA and the RTA), China (sixth in the RCA and fifth in the RTA), and Indonesia (fifth in the RCA and fourth in the RTA). Philippines possess a weak competitive advantage in trade with RCEP countries which matches with earlier revealed relative trade advantage.

Overall, for major traders, the LI demonstrates competitive disadvantages more often than for smaller countries. In many [VNM;j] and [IDN;j] pairs, as well as in several [CHN;j], [THA;j], and [NZL;j] pairs, a potential strong or average advantage turns to a weak one. Conversely, most of smaller economies (Cambodia, Singapore, Brunei Darussalam), as well as large fish trading nations where RTAs were not detected previously (Japan, South Korea, Malaysia, and Australia), now show competitive advantages. Brunei Darussalam, Cambodia, and Lao PDR, for which the RTA previously showed a disadvantage across most of $[i ; j]$ pairs, could obtain potential competitiveness in trade with Australia $\left(p_{4}, p_{5}\right)$, China $\left(p_{2}, p_{3}, p_{5}\right)$, Indonesia $\left(p_{2}\right)$, Malaysia $\left(p_{5}\right)$, Vietnam $\left(p_{3}, p_{5}\right)$, and Singapore $\left(p_{2}, p_{3}\right)$. Bigger traders like Japan, South Korea, and Australia demonstrate competitive advantages in trade between themselves (Japan and South Korea in all categories $p_{2-5}$, Australia and Japan in $p_{3}$ and $p_{5}$ ), as well as in trade in value-added prepared and preserved fish and aquatic invertebrates $\left(p_{5}\right)$ with China and Vietnam (Australia), crustaceans and mollusks $\left(p_{4}\right)$ with China and New Zealand (Japan), salted and smoked fish $\left(p_{3}\right)$ with Singapore (South Korea), and fresh fish $\left(p_{2}\right)$ with Cambodia, Philippines, and Malaysia (Australia and Japan).

\subsection{Advantages and Potentials}

Therefore, we receive a picture of diverse relationships between higher and lower potentials and stronger and weaker advantages across 210 pairs of countries. By merging 
potentials and advantages into one matrix, we detect $[i ; j]$ and $[i ; r]$ pairs in which untapped trade potentials coincide with competitive advantages (Table 5).

Table 5. Degrees of trade potentials and advantages of RCEP countries in trade in fish, crustaceans, and mollusks $\left(p_{1}\right)$ in 2000-2019.

\begin{tabular}{ccccccccccccccccc}
\hline Countries & AUS & BRN & KHM & CHN & IDN & JPN & LAO & MYS & MMR & NZL & PHL & SGP & KOR & THA & VNM & RCEP \\
\hline AUS & & W & D & W & D & A & A & D & D & D & D & A & D & D & S & D \\
BRN & W & & D & W & W & W & A & D & D & D & D & D & W & W & D & D \\
KHM & S & A & & A & D & W & S & A & W & D & D & A & A & W & D & D \\
CHN & W & D & D & & D & S & A & S & D & D & W & A & S & W & D & S \\
IDN & W & A & A & W & & S & A & W & D & W & D & A & A & S & S & S \\
JPN & D & A & A & D & D & & A & W & D & W & D & W & W & W & D & D \\
LAO & D & A & D & D & D & D & & D & A & D & W & W & A & D & D & D \\
MYS & A & S & W & D & D & A & A & & D & D & D & A & A & D & W & D \\
MMR & S & S & A & S & W & S & A & S & & W & S & S & S & W & S & S \\
NZL & S & S & S & S & D & S & S & W & D & & D & S & W & W & W & S \\
PHL & A & S & A & D & W & S & A & A & D & W & & A & A & A & D & A \\
SGP & D & A & W & D & D & D & A & D & D & D & D & & W & D & D & D \\
KOR & A & A & D & D & D & A & W & D & D & S & D & W & & W & D & D \\
THA & S & S & W & D & D & S & A & A & D & W & D & A & W & W & S \\
VNM & S & S & A & W & D & S & A & W & D & W & W & S & S & W & S \\
\hline
\end{tabular}

Note: Country abbreviations (AUS = Australia; BRN = Brunei Darussalam; KHM = Cambodia; CHN = China; IDN = Indonesia; JPN = Japan; LAO = Lao PDR; MYS = Malaysia; MMR = Myanmar; NZL = New Zealand; PHL = Philippines; SGP = Singapore; KOR = South Korea; THA = Thailand; VNM = Vietnam); green cells = high trade potential; blue cells = trade potential above average; yellow cells = trade potential below average; red cells = low trade potential; $\mathrm{S}=$ strong advantage; $\mathrm{A}=$ average advantage; $\mathrm{W}=$ weak advantage; $\mathrm{D}=$ disadvantage. Source: Authors' development.

Despite the fact that most of $[i ; j]$ pairs demonstrate high potentials for an increase in bilateral trade in fish, crustaceans, and mollusks $\left(p_{1}\right)$, we see that in many cases, indicative potentials are not backed up by competitive, trade, or even comparative advantages. Conversely, either strong or average competitive advantages are identified in pairs where the indicative potential is below average (for example, [NZL;CHN], [KOR;NZL], and [VNM;JPN] pairs) or even low ([THA;LAO] pair). Similar discrepancies between the degrees of trade potentials and strengths of competitive advantages are observed in trade in fresh, chilled, and frozen fish $\left(p_{2}\right)$ (Appendix C, Table A11), dried, salted, and smoked fish $\left(p_{3}\right)$ (Appendix C, Table A12), crustaceans and mollusks $\left(p_{4}\right)$ (Appendix C, Table A13), and prepared and preserved aquatic invertebrates $\left(p_{5}\right)$ (Appendix C, Table A14). The per-country results and implications of these findings are further detailed in the Section 4.

\section{Discussion}

\subsection{Optimization of Trade Potentials and Advantages}

In light of the revealed diverse relationships between indicative trade potentials and the three types of advantages that differentiate between bigger and smaller RCEP countries, we discuss the findings separately for major trading nations (the high portion in the overall intraregional trade turnover of fish and seafood products), countries that specialize in trade in fish and fishery products (high share of fish products in the total national trade turnover with RCEP countries), smaller economies (low contribution to the intraregional trade in fish and seafood products), and other economies of the region (average parameters of both the share in the intraregional trade in fish and the share of fish products in foreign trade with RCEP countries).

Across these four groups of countries and individual economies, we established recommendations for prospective (trade should be increased), disadvantageous (trade should be decreased), and neutral (no changes in actual trade required) trading destinations and products (Table 6). The grouping is based on different combinations of trade potentials and advantages:

- Advantageous destinations/products: high trade potential + strong, or average, or weak advantage; trade potential above average + strong, or average, or weak advantage. 
- Disadvantageous destinations/products: trade potential above average + disadvantage; trade potential below average + weak advantage or disadvantage; low trade potential + weak advantage or disadvantage.

- Neutral destinations/products: high trade potential + disadvantage; trade potential below average + strong or average advantage; low trade potential + strong or average advantage.

Table 6. Optimization of trade potentials and advantages in the intra-RCEP trade: destinations and products.

\begin{tabular}{|c|c|c|c|}
\hline $\begin{array}{c}\text { Groups/ } \\
\text { Countries }\end{array}$ & $\begin{array}{c}\text { Advantage, } \\
\text { Destinations (Products) * }\end{array}$ & $\begin{array}{c}\text { Disadvantage, } \\
\text { Destinations (Products) ** }\end{array}$ & $\begin{array}{c}\text { Neutral, } \\
\text { Destinations (Products) *** }\end{array}$ \\
\hline \multicolumn{4}{|l|}{ Group 1} \\
\hline $\mathrm{CHN}$ & $\begin{array}{c}\text { AUS }\left(p_{2-5}\right), \operatorname{BRN}\left(p_{5}\right), \operatorname{KHM}\left(p_{3}\right. \\
\left.p_{5}\right), \mathrm{JPN}\left(p_{4}\right), \mathrm{LAO}\left(p_{2-5}\right), \mathrm{MYS}\left(p_{2}\right. \\
\left.p_{3}\right), \mathrm{NZL}\left(p_{5}\right), \operatorname{SGP}\left(p_{2}, p_{4}, p_{5}\right) \\
\text { THA }\left(p_{2}, p_{3}\right)\end{array}$ & $\begin{array}{c}\mathrm{IDN}\left(p_{2}, p_{4}, p_{5}\right), \operatorname{MMR}\left(p_{2-5}\right), \mathrm{NZL}\left(p_{2},\right. \\
\left.p_{4}\right), \\
\operatorname{PHL}\left(p_{3}, p_{5}\right), \underset{ }{\mathrm{THA}}\left(p_{4}, p_{5}\right), \mathrm{VNM} \\
\left(p_{2-5}\right)\end{array}$ & $\begin{array}{c}\mathrm{BRN}\left(p_{2-4}\right), \mathrm{KHM}\left(p_{2}, p_{4}\right), \mathrm{IDN}\left(p_{3}\right), \\
\mathrm{JPN}\left(p_{2}, p_{3}, p_{5}\right), \mathrm{MYS}\left(p_{4}, p_{5}\right), \mathrm{NZL} \\
\left(p_{3}\right), \mathrm{PHL}\left(p_{2}, p_{4}\right), \operatorname{SGP}\left(p_{3}\right), \mathrm{KOR} \\
\left(p_{2-5}\right)\end{array}$ \\
\hline JPN & $\begin{array}{c}\operatorname{AUS}\left(p_{5}\right), \operatorname{BRN}\left(p_{2}, p_{3}, p_{5}\right), \mathrm{KHM} \\
\left(p_{2-5}\right), \mathrm{LAO}\left(p_{2-5}\right) \\
\operatorname{MYS}\left(p_{2}, p_{3}, p_{5}\right), \mathrm{NZL}\left(p_{4}, p_{5}\right) \\
\operatorname{SGP}\left(p_{2-5}\right)\end{array}$ & $\begin{array}{l}\operatorname{AUS}\left(p_{2}, p_{4}\right), \operatorname{CHN}\left(p_{2-5}\right), \operatorname{IDN}\left(p_{2-5}\right) \\
\operatorname{MMR}\left(p_{2-5}\right), \operatorname{NZL}\left(p_{2}, p_{3}\right), \operatorname{PHL}\left(p_{2-5}\right) \\
\operatorname{KOR}\left(p_{2-5}\right), \operatorname{THA}\left(p_{2-5}\right), \operatorname{VNM}\left(p_{2-5}\right)\end{array}$ & $\operatorname{AUS}\left(p_{3}\right), \operatorname{BRN}\left(p_{4}\right), \operatorname{MYS}\left(p_{4}\right)$ \\
\hline KOR & $\begin{array}{c}\operatorname{AUS}\left(p_{3-5}\right), \operatorname{BRN}\left(p_{2}, p_{3}, p_{5}\right), \mathrm{KHM} \\
\left(p_{3}, p_{5}\right), \mathrm{LAO}\left(p_{2-5}\right) \\
\operatorname{MYS}\left(p_{3}\right), \operatorname{MMR}\left(p_{3}\right), \mathrm{NZL}\left(p_{3}, p_{4}\right), \\
\operatorname{PHL}\left(p_{2}\right), \operatorname{SGP}\left(p_{3-5}\right), \text { THA }\left(p_{2}\right)\end{array}$ & $\begin{array}{c}\mathrm{CHN}\left(p_{2-5}\right), \mathrm{IDN}\left(p_{4}\right), \mathrm{JPN}\left(p_{3}\right), \mathrm{MMR} \\
\left(p_{2}, p_{4}\right), \\
\mathrm{PHL}\left(p_{3-5}\right), \mathrm{THA}\left(p_{4}\right), \mathrm{VNM}\left(p_{2-5}\right)\end{array}$ & $\begin{array}{c}\operatorname{AUS}\left(p_{2}\right), \operatorname{BRN}\left(p_{4}\right), \operatorname{KHM}\left(p_{2}, p_{4}\right), \\
\operatorname{IDN}\left(p_{2}, p_{3}, p_{5}\right), \operatorname{JPN}\left(p_{2}, p_{4}, p_{5}\right), \\
\operatorname{MYS}\left(p_{2}, p_{4}, p_{5}\right), \operatorname{MMR}\left(p_{5}\right), \\
\operatorname{NZL}\left(p_{2}, p_{5}\right), \operatorname{SGP}\left(p_{2}\right), \operatorname{THA}\left(p_{3}, p_{5}\right)\end{array}$ \\
\hline \multicolumn{4}{|l|}{ Group 2} \\
\hline MMR & $\begin{array}{c}\text { AUS }\left(p_{2-5}\right), \text { BRN }\left(p_{2-5}\right), \text { KHM } \\
\left(p_{3-5}\right), \text { CHN }\left(p_{2-5}\right), \mathrm{IDN}\left(p_{2-4}\right), \\
\text { JPN }\left(p_{2}, p_{3}, p_{5}\right), \mathrm{LAO}\left(p_{2-5}\right), \mathrm{MYS} \\
\left(p_{2-5}\right), \mathrm{NZL}\left(p_{2}, p_{4}, p_{5}\right), \\
\text { PHL }\left(p_{2-5}\right), \text { SGP }\left(p_{2-5}\right), \mathrm{KOR} \\
\left(p_{2-5}\right), \text { THA }\left(p_{2-4}\right), \mathrm{VNM}\left(p_{2-5}\right)\end{array}$ & & $\begin{array}{c}\mathrm{KHM}\left(p_{2}\right), \operatorname{IDN}\left(p_{5}\right), \mathrm{JPN}\left(p_{4}\right), \mathrm{NZL} \\
\left(p_{3}\right), \mathrm{THA}\left(p_{5}\right)\end{array}$ \\
\hline THA & $\begin{array}{c}\text { AUS }\left(p_{2-5}\right), \operatorname{BRN}\left(p_{2-5}\right), \mathrm{CHN}\left(p_{3}\right. \\
\left.p_{4}\right), \mathrm{IDN}\left(p_{3}\right), \mathrm{JPN}\left(p_{3}, p_{5}\right), \mathrm{MYS} \\
\left(p_{2}, p_{3}, p_{5}\right), \mathrm{NZL}\left(p_{3}, p_{4}\right), \mathrm{SGP} \\
\left(p_{2-5}\right), \mathrm{KOR}\left(p_{3-5}\right)\end{array}$ & $\begin{array}{c}\operatorname{KHM}\left(p_{2-4}\right), \operatorname{CHN}\left(p_{2}\right), \operatorname{IDN}\left(p_{2}, p_{4},\right. \\
\left.p_{5}\right), \\
\operatorname{MMR}\left(p_{2-5}\right), \mathrm{NZL}\left(p_{2}, p_{5}\right), \operatorname{PHL}\left(p_{3},\right. \\
\left.p_{5}\right), \operatorname{VNM}\left(p_{2-5}\right)\end{array}$ & $\begin{array}{c}\operatorname{KHM}\left(p_{5}\right), \operatorname{CHN}\left(p_{5}\right), \operatorname{JPN}\left(p_{2}, p_{4}\right), \\
\mathrm{LAO}\left(p_{2-5}\right), \operatorname{MYS}\left(p_{4}\right), \operatorname{PHL}\left(p_{2}, p_{4}\right), \\
\operatorname{KOR}\left(p_{2}\right)\end{array}$ \\
\hline VNM & $\begin{array}{c}\text { AUS }\left(p_{2-5}\right), \operatorname{BRN}\left(p_{2-5}\right), \mathrm{KHM}\left(p_{3}\right. \\
\left.p_{5}\right), \mathrm{CHN}\left(p_{2}, p_{5}\right), \\
\operatorname{IDN}\left(p_{3}, p_{5}\right), \mathrm{JPN}\left(p_{2}, p_{3}, p_{5}\right), \mathrm{LAO} \\
\left(p_{2-5}\right), \mathrm{MYS}\left(p_{2-5}\right), \\
\mathrm{NZL}\left(p_{2-5}\right), \mathrm{PHL}\left(p_{2-4}\right), \mathrm{SGP}\left(p_{2-5}\right) \\
\mathrm{KOR}\left(p_{2-5}\right), \mathrm{THA}\left(p_{2-5}\right)\end{array}$ & $\operatorname{KHM}\left(p_{4}\right), \operatorname{IDN}\left(p_{4}\right), \operatorname{MMR}\left(p_{3}, p_{4}\right)$ & $\begin{array}{c}\mathrm{KHM}\left(p_{2}\right), \operatorname{CHN}\left(p_{3}, p_{4}\right), \operatorname{IDN}\left(p_{2}\right), \\
\operatorname{JPN}\left(p_{4}\right), \\
\operatorname{MMR}\left(p_{2}, p_{5}\right), \operatorname{PHL}\left(p_{5}\right)\end{array}$ \\
\hline \multicolumn{4}{|l|}{ Group 3} \\
\hline AUS & $\begin{array}{l}\mathrm{BRN}\left(p_{2-5}\right), \operatorname{KHM}\left(p_{5}\right), \mathrm{CHN}\left(p_{2}\right) \\
\mathrm{JPN}\left(p_{4}, p_{5}\right), \mathrm{LAO}\left(p_{2-5}\right), \operatorname{PHL}\left(p_{2}\right) \\
\operatorname{SGP}\left(p_{2-5}\right), \operatorname{KOR}\left(p_{2}\right)\end{array}$ & $\begin{array}{c}\operatorname{KHM}\left(p_{3}\right), \operatorname{CHN}\left(p_{4}, p_{5}\right), \operatorname{IDN}\left(p_{4}\right) \\
\operatorname{JPN}\left(p_{3}\right), \\
\operatorname{MMR}\left(p_{4}, p_{5}\right), \operatorname{NZL}\left(p_{2-4}\right), \operatorname{PHL}\left(p_{3}\right), \\
\operatorname{THA}\left(p_{4}, p_{5}\right), \operatorname{VNM}\left(p_{2}, p_{5}\right)\end{array}$ & $\begin{array}{c}\mathrm{KHM}\left(p_{2}, p_{4}\right), \mathrm{CHN}\left(p_{3}\right), \mathrm{IDN}\left(p_{2},\right. \\
\left.p_{3}, p_{5}\right), \mathrm{JPN}\left(p_{2}\right), \mathrm{MYS}\left(p_{2-5}\right), \mathrm{MMR} \\
\left(p_{3}\right), \mathrm{NZL}\left(p_{5}\right), \mathrm{PHL}\left(p_{4}, p_{5}\right), \mathrm{KOR} \\
\left(p_{3-5}\right), \mathrm{THA}\left(p_{2}, p_{3}\right), \operatorname{VNM}\left(p_{3}, p_{4}\right)\end{array}$ \\
\hline IDN & $\begin{array}{c}\text { AUS }\left(p_{2-5}\right), \operatorname{BRN}\left(p_{2-5}\right), \mathrm{KHM} \\
\left(p_{2-5}\right), \mathrm{CHN}\left(p_{2-4}\right), \mathrm{JPN}\left(p_{2}\right), \mathrm{LAO} \\
\left(p_{2-5}\right), \operatorname{MYS}\left(p_{2}, p_{4}\right), \mathrm{MMR}\left(p_{5}\right), \\
\mathrm{NZL}\left(p_{3-5}\right), \operatorname{SGP}\left(p_{2-5}\right), \mathrm{KOR}\left(p_{2-5}\right) \\
\mathrm{THA}\left(p_{2}, p_{4}, p_{5}\right), \operatorname{VNM}\left(p_{2}, p_{5}\right)\end{array}$ & $\operatorname{MMR}\left(p_{3}, p_{4}\right), \operatorname{THA}\left(p_{3}\right), \operatorname{VNM}\left(p_{3}, p_{4}\right)$ & $\begin{array}{c}\mathrm{CHN}\left(p_{5}\right), \mathrm{JPN}\left(p_{3-5}\right), \operatorname{MYS}\left(p_{3}, p_{5}\right) \\
\operatorname{MMR}\left(p_{2}\right), \text { NZL }\left(p_{2}\right), \operatorname{PHL}\left(p_{2-5}\right)\end{array}$ \\
\hline MYS & $\begin{array}{l}\text { AUS }\left(p_{2-5}\right), \operatorname{BRN}\left(p_{2-5}\right), \mathrm{KHM} \\
\left(p_{3-5}\right), \operatorname{IDN}\left(p_{3}\right), \mathrm{JPN}\left(p_{2}, p_{4}, p_{5}\right) \\
\mathrm{LAO}\left(p_{2-5}\right), \mathrm{NZL}\left(p_{5}\right), \operatorname{PHL}\left(p_{2}\right) \\
\quad \operatorname{SGP}\left(p_{2-5}\right), \operatorname{KOR}\left(p_{2-5}\right)\end{array}$ & $\begin{array}{c}\mathrm{CHN}\left(p_{2}, p_{4}\right), \operatorname{IDN}\left(p_{2}, p_{4}\right), \mathrm{MMR} \\
\left(p_{2-5}\right), \\
\operatorname{THA}\left(p_{2}, p_{3}\right), \operatorname{VNM}\left(p_{2}, p_{3}, p_{5}\right)\end{array}$ & $\begin{array}{c}\mathrm{KHM}\left(p_{2}\right), \operatorname{CHN}\left(p_{3}, p_{5}\right), \operatorname{IDN}\left(p_{5}\right), \\
\operatorname{JPN}\left(p_{3}\right), \\
\operatorname{NZL}\left(p_{2-4}\right), \operatorname{PHL}\left(p_{3-5}\right), \operatorname{THA}\left(p_{4},\right. \\
\left.p_{5}\right), \operatorname{VNM}\left(p_{4}\right)\end{array}$ \\
\hline
\end{tabular}


Table 6. Cont.

\begin{tabular}{|c|c|c|c|}
\hline $\begin{array}{l}\text { Groups/ } \\
\text { Countries }\end{array}$ & $\begin{array}{c}\text { Advantage, } \\
\text { Destinations (Products) * }\end{array}$ & $\begin{array}{c}\text { Disadvantage, } \\
\text { Destinations (Products) ** }\end{array}$ & $\begin{array}{c}\text { Neutral, } \\
\text { Destinations (Products) } * * *\end{array}$ \\
\hline NZL & $\begin{array}{c}\text { AUS }\left(p_{3-5}\right), \operatorname{BRN}\left(p_{2-5}\right), \mathrm{KHM} \\
\left(p_{2-5}\right), \mathrm{CHN}\left(p_{2}, p_{5}\right), \mathrm{JPN}\left(p_{2-5}\right) \\
\mathrm{LAO}\left(p_{2-5}\right), \mathrm{MYS}\left(p_{2-5}\right), \mathrm{MMR}\left(p_{3}\right) \\
\text { PHL }\left(p_{2}, p_{4}\right), \operatorname{SGP}\left(p_{2-5}\right), \mathrm{KOR} \\
\left(p_{2-5}\right), \mathrm{THA}\left(p_{2}, p_{4}, p_{5}\right), \operatorname{VNM}\left(p_{2}\right)\end{array}$ & PHL $\left(p_{3}\right)$ & $\begin{array}{c}\operatorname{AUS}\left(p_{2}\right), \mathrm{CHN}\left(p_{3}, p_{4}\right), \mathrm{IDN}\left(p_{2-5}\right), \\
\operatorname{MMR}\left(p_{2}, p_{4}, p_{5}\right), \text { PHL }\left(p_{5}\right), \mathrm{THA} \\
\left(p_{3}\right), \operatorname{VNM}\left(p_{3-5}\right)\end{array}$ \\
\hline SGP & $\begin{array}{l}\text { AUS }\left(p_{3}, p_{5}\right), \mathrm{BRN}\left(p_{2-5}\right), \mathrm{KHM} \\
\left(p_{3-5}\right), \mathrm{CHN}\left(p_{3}\right), \mathrm{IDN}\left(p_{5}\right), \mathrm{JPN} \\
\left(p_{2}, p_{3}\right), \mathrm{LAO}\left(p_{2-5}\right), \mathrm{MYS}\left(p_{3}\right) \\
\mathrm{MMR}\left(p_{5}\right), \mathrm{PHL}\left(p_{2}, p_{5}\right), \mathrm{KOR} \\
\left(p_{2-5}\right), \mathrm{THA}\left(p_{2}, p_{3}\right), \operatorname{VNM}\left(p_{2}\right)\end{array}$ & $\begin{array}{c}\operatorname{AUS}\left(p_{4}\right), \mathrm{CHN}\left(p_{2}, p_{4}, p_{5}\right), \mathrm{IDN}\left(p_{2-4}\right) \\
\mathrm{JPN}\left(p_{4}\right), \mathrm{MYS}\left(p_{2}, p_{4}, p_{5}\right), \mathrm{MMR} \\
\left(p_{2-4}\right), \mathrm{NZL}\left(p_{3}\right), \mathrm{PHL}\left(p_{3}\right), \mathrm{THA}\left(p_{4}\right. \\
\left.p_{5}\right), \operatorname{VNM}\left(p_{3-5}\right)\end{array}$ & $\begin{array}{c}\operatorname{AUS}\left(p_{2}\right), \operatorname{KHM}\left(p_{2}\right), \mathrm{JPN}\left(p_{5}\right), \mathrm{NZL} \\
\left(p_{2}, p_{4}, p_{5}\right), \mathrm{PHL}\left(p_{4}\right)\end{array}$ \\
\hline \multicolumn{4}{|l|}{ Group 4} \\
\hline BRN & $\begin{array}{c}\operatorname{AUS}\left(p_{4}, p_{5}\right), \operatorname{KHM}\left(p_{3-5}\right), \mathrm{IDN} \\
\left(p_{2}\right), \mathrm{JPN}\left(p_{2}, p_{4}\right), \mathrm{LAO}\left(p_{2-5}\right) \\
\operatorname{MMR}\left(p_{3}, p_{5}\right), \operatorname{PHL}\left(p_{3}\right), \operatorname{KOR}\left(p_{3}\right. \\
\left.p_{4}\right), \mathrm{THA}\left(p_{2}\right), \operatorname{VNM}\left(p_{4}\right)\end{array}$ & $\begin{array}{c}\text { AUS }\left(p_{3}\right), \mathrm{CHN}\left(p_{2}, p_{3}, p_{5}\right), \mathrm{IDN}\left(p_{5}\right) \\
\mathrm{JPN}\left(p_{5}\right), \mathrm{MYS}\left(p_{2-5}\right), \mathrm{MMR}\left(p_{4}\right), \mathrm{NZL} \\
\left(p_{2}, p_{5}\right), \mathrm{PHL}\left(p_{5}\right), \operatorname{SGP}\left(p_{2-5}\right), \mathrm{KOR} \\
\left(p_{5}\right), \mathrm{THA}\left(p_{3}, p_{5}\right) \\
\operatorname{VNM}\left(p_{2}, p_{3}, p_{5}\right)\end{array}$ & $\begin{array}{c}\operatorname{AUS}\left(p_{2}\right), \operatorname{KHM}\left(p_{2}\right), \operatorname{CHN}\left(p_{4}\right), \operatorname{IDN} \\
\left(p_{3}, p_{4}\right), \\
\operatorname{JPN}\left(p_{3}\right), \operatorname{MMR}\left(p_{2}\right), \operatorname{NZL}\left(p_{3}, p_{4}\right) \\
\operatorname{PHL}\left(p_{2}, p_{4}\right), \operatorname{KOR}\left(p_{2}\right), \operatorname{THA}\left(p_{4}\right)\end{array}$ \\
\hline KHM & $\begin{array}{c}\text { AUS }\left(p_{2-5}\right), \operatorname{BRN}\left(p_{2-5}\right), \mathrm{CHN}\left(p_{2}\right. \\
\left.p_{3}\right), \mathrm{IDN}\left(p_{2}\right), \mathrm{JPN}\left(p_{2}, p_{4}\right), \mathrm{LAO} \\
\left(p_{2-5}\right), \operatorname{MYS}\left(p_{2}, p_{3}, p_{5}\right), \mathrm{MMR} \\
\left(p_{3-5}\right), \mathrm{NZL}\left(p_{2}\right), \\
\text { PHL }\left(p_{2}\right), \operatorname{SGP}\left(p_{2-4}\right), \operatorname{KOR}\left(p_{2}, p_{4}\right)\end{array}$ & $\begin{array}{c}\mathrm{CHN}\left(p_{5}\right), \mathrm{JPN}\left(p_{5}\right), \mathrm{THA}\left(p_{4}, p_{5}\right) \\
\operatorname{VNM}\left(p_{2}, p_{4}, p_{5}\right)\end{array}$ & $\begin{array}{r}\mathrm{CHN}\left(p_{4}\right), \operatorname{IDN}\left(p_{3-5}\right), \operatorname{JPN}\left(p_{3}\right), \\
\operatorname{MYS}\left(p_{4}\right), \\
\operatorname{MMR}\left(p_{2}\right), \operatorname{NZL}\left(p_{3-5}\right), \operatorname{PHL}\left(p_{3-5}\right), \\
\operatorname{SGP}\left(p_{5}\right), \\
\operatorname{KOR}\left(p_{3}, p_{5}\right), \operatorname{THA}\left(p_{2}, p_{3}\right), \operatorname{VNM} \\
\left(p_{3}\right)\end{array}$ \\
\hline LAO & $\begin{array}{c}\text { AUS }\left(p_{2}, p_{3}\right), \operatorname{BRN}\left(p_{2-4}\right), \mathrm{KHM} \\
\left(p_{3}, p_{4}\right), \mathrm{CHN}\left(p_{2}\right), \mathrm{IDN}\left(p_{3}\right), \mathrm{JPN} \\
\left(p_{3}\right), \mathrm{MYS}\left(p_{2}, p_{3}\right), \operatorname{MMR}\left(p_{2-4}\right) \\
\mathrm{NZL}\left(p_{2}, p_{3}\right), \operatorname{PHL}\left(p_{2-4}\right), \operatorname{SGP}\left(p_{2}\right) \\
\operatorname{KOR}\left(p_{4}\right)\end{array}$ & $\begin{array}{c}\text { AUS }\left(p_{4}\right), \operatorname{KHM}\left(p_{2}\right), \operatorname{CHN}\left(p_{3}, p_{5}\right) \\
\operatorname{IDN}\left(p_{4}\right), \\
\operatorname{JPN}\left(p_{5}\right), \operatorname{NZL}\left(p_{4}\right), \operatorname{SGP}\left(p_{3}\right), \mathrm{KOR} \\
\left(p_{3}\right), \\
\text { THA }\left(p_{2-5}\right), \operatorname{VNM}\left(p_{2-5}\right)\end{array}$ & $\begin{array}{c}\text { AUS }\left(p_{5}\right), \operatorname{BRN}\left(p_{5}\right), \operatorname{KHM}\left(p_{5}\right), \\
\text { CHN }\left(p_{4}\right), \\
\operatorname{IDN}\left(p_{5}\right), \mathrm{JPN}\left(p_{2}, p_{4}\right), \operatorname{MYS}\left(p_{4}, p_{5}\right), \\
\operatorname{MMR}\left(p_{5}\right), \mathrm{NZL}\left(p_{5}\right), \operatorname{PHL}\left(p_{5}\right), \mathrm{SGP} \\
\left(p_{4}, p_{5}\right), \operatorname{KOR}\left(p_{2}, p_{5}\right)\end{array}$ \\
\hline PHL & $\begin{array}{c}\text { AUS }\left(p_{2-5}\right), \mathrm{BRN}\left(p_{2-5}\right), \mathrm{KHM} \\
\left(p_{2-5}\right), \mathrm{CHN}\left(p_{3}\right), \mathrm{IDN}\left(p_{3-5}\right), \mathrm{JPN} \\
\left(p_{5}\right), \mathrm{LAO}\left(p_{2-5}\right), \mathrm{MYS}\left(p_{3-5}\right) \\
\mathrm{MMR}\left(p_{3}\right), \mathrm{NZL}\left(p_{3}, p_{5}\right), \mathrm{SGP}\left(p_{2}\right. \\
\left.p_{4}, p_{5}\right), \mathrm{KOR}\left(p_{3-5}\right), \mathrm{THA}\left(p_{2-5}\right) \\
\operatorname{VNM}\left(p_{4}, p_{5}\right)\end{array}$ & $\mathrm{CHN}\left(p_{2}\right), \operatorname{VNM}\left(p_{2}\right)$ & $\begin{array}{l}\mathrm{CHN}\left(p_{4}, p_{5}\right), \operatorname{IDN}\left(p_{2}\right), \operatorname{JPN}\left(p_{2-4}\right) \\
\operatorname{MYS}\left(p_{2}\right), \operatorname{MMR}\left(p_{2}, p_{4}, p_{5}\right), \mathrm{NZL} \\
\left(p_{4}\right), \operatorname{SGP}\left(p_{3}\right), \operatorname{KOR}\left(p_{2}\right), \operatorname{VNM}\left(p_{3}\right)\end{array}$ \\
\hline
\end{tabular}

Note: Groups $(1=$ large contribution to the total intra-RCEP trade turnover of fish and seafood products; 2 = high share of fish products in the total national trade turnover with RCEP countries; 3 = low contribution to the intra-RCEP trade turnover of fish and seafood products; 4 = other countries); country abbreviations (AUS = Australia; BRN = Brunei Darussalam; $\mathrm{KHM}=\mathrm{Cambodia}$; $\mathrm{CHN}=\mathrm{China}$; IDN = Indonesia; JPN = Japan; LAO = Lao PDR; MYS = Malaysia; MMR = Myanmar; NZL = New Zealand; PHL = Philippines; SGP = Singapore; KOR = South Korea; THA $=$ Thailand; VNM $=$ Vietnam); ${ }^{*}=$ trade in $p_{n}$ products with $j$ destinations can be increased; ${ }^{* *}=$ trade in $p_{n}$ products with $j$ destinations should be decreased; ${ }^{* *}=$ no need for substantial changes in current trade in $p_{n}$ products with $j$ destinations. Source: Authors' development.

The key takeaways from Table 6 can be summarized as follows:

- Major traders have made good use of their potentials and advantages in trade with other countries of the region, as well as between themselves. There are untapped advantages in trade in high-priced fishery products with smaller economies (bivalves and fresh and frozen fish $\left(p_{2}\right)$ and prepared and preserved fishery products $\left(p_{5}\right)$ (further discussed in Section 4.2).

- Countries that specialize in fishery obtain the strongest advantages in trade with higher-income RCEP economies to the extent that they have become overdependent on Chinese, Japanese, and Korean markets. Group 2 economies can gain from the diversification of the intra-RCEP trade by increasing exports of fresh, chilled, and frozen fish $\left(p_{2}\right)$ and crustaceans, mollusks, and aquatic invertebrates $\left(p_{4}\right)$ all over the region (further discussed in Section 4.3). 
- Countries that make a minor contribution to the intra-RCEP trade in fish possess weaker advantages compared to both Group 1 and Group 2 traders. There is room to expand trade with the smallest RCEP member states, as well as to increase supplies of processed fishery products to higher-income economies (further discussed in Section 4.4).

- Smallest traders obtain the weakest advantages, while the fishery sector plays a crucial role in the economic development of and social stability in the poor rural and coastal communities. For these countries, the mismatches between high untapped trade potentials and disadvantages or weak competitive advantages are particularly common (further discussed in Section 4.5).

\subsection{Major Traders: China, Japan, South Korea}

In confirmation of the earlier addressed asymmetries in the intra-RCEP trade, we see that bigger higher-income traders such as China, Japan, and South Korea make the greatest contribution to the intra-regional fish trade. Compared to less developed and smaller economies, the ITP values in bigger traders demonstrate a higher degree of utilization of trade potential. Commonly, in $[i ; j]$ pairs with low trade potentials, a Group 1 country $i$ experiences a disadvantage in trade with a country $j$ or exhibits a weak advantage (ChinaPhilippines, Japan-Thailand, Japan-New Zealand).

Group 1 countries have almost maximized trade values between themselves. In this situation, further liberalization of fish trade would not result in a significant transformation of either trade flows or competitive advantages of the three nations-a confrontation with earlier findings of negative effects of South Korea-China and South Korea-Japan free trade agreements made by Kim [116]. In all bilateral pairs within Group 1, trade potential is below average (even though China still enjoys a strong advantage in trade with Japan and South Korea). China's advantages are particularly prominent in trade in crustaceans, mollusks, and aquatic invertebrates $\left(p_{4}\right)$. According to Einarsson and Óladóttir [35] and Luo et al. [72], this advantage can be attributed to China's aquaculture sector that is larger than Japan's and South Korea's aquaculture sectors combined.

From an optimization standpoint, a situation when actual trade is close to a potential value or even exceeds it could be addressed by diversification towards destinations where untapped gains are higher. Dunmore [112] admitted that such transformations could arise in an undistorted trade pattern based on differences in advantages between two countries. However, bilateral flows exist in the broader economic context, they are contributed by many factors aside from advantages or relative efficiencies. While free trade agreements in Asia have increased bilateral trade, they have also contributed to widening deficits in smaller countries' trade with higher-income Group 1 economies [117-119]. Governments all over the world have been widely applying conventional customs measures such as tariffs and non-tariff regulations to cope with deficits, optimize trade balance, and redirect trade flows [120-123]. Still, there is no economic reason why trade between any pair of countries should balance [124], as well as why the potential-actual trade gap should be reduced by any means for the sake of optimization. Previously in ASEAN and now in RCEP, tariffs are no longer dominant as they have been reduced to rather modest levels and are not widely divergent [125]. Therefore, for RCEP countries, the optimization of bilateral trade should focus on the improvement of efficiency gains rather than rerouting existing trade. These should include the most pressing issues that hinder the competitiveness of smaller traders in the intra-RCEP fisheries trade, such as diversification of the exportproduct mix, production and logistics costs, quality assurance and licensing, post-harvest losses, small-scale fisheries, and infrastructure.

According to our projections, South Korea can potentially increase trade in fresh fish $\left(p_{2}\right)$ with Thailand, Philippines, Lao PDR, and Brunei Darussalam, in dried and salted fish $\left(p_{3}\right)$ with Cambodia, Malaysia, and Myanmar, and in crustaceans and mollusks $\left(p_{4}\right)$ with Australia and New Zealand. As against the insignificant potential of South KoreaSingapore trade earlier demonstrated by Choi and Choi [119], we see an advantage in trade 
in dried and salted fish $\left(p_{3}\right)$ and prepared and preserved aquatic invertebrates $\left(p_{5}\right)$ in this pair of countries. Japan has been gradually losing competitive advantages in trade with Group 1 countries since the 2000s. According to the FAO's projection [12], fish production in Japan will fall by $6.7 \%$ in 2030 compared to 2018. However, amid losing advantages in the global market, we see that Japan has the potential to improve its position in the intraRCEP trade by increasing supplies to smaller and less developed economies of the region (Brunei Darussalam, Cambodia, and Lao PDR). Also, processed value-added prepared and preserved fishery products $\left(p_{5}\right)$ from Japan possess a competitive advantage in Australia and New Zealand.

\subsection{High Specialization in Fish and Seafood Trade: Myanmar, Thailand, Vietnam}

In support of previous results of Hoang [70], Khai et al. [87], Teh and Pauly [29], and Kurien [126], we reveal that Group 2 countries have a huge potential to increase their contribution to the intra-RCEP trade. This can happen due to a favorable potential/advantage ratio in trade in fresh, chilled, and frozen fish $\left(p_{2}\right)$ (major tuna processing and export facilities along with a long-distance tuna fleet in Thailand [12]) and crustaceans, mollusks, and aquatic invertebrates $\left(p_{4}\right)$ (supplies of squid and cuttlefish by Vietnam [12]). Our findings of a high potential of Group 2 countries to increase their trade in various types of fish and seafood products agree with the FAO's projections [10] of the most substantial growth of the value of fish trade in Vietnam and Thailand by 2030 (by 39.8\% and 20.6\%, respectively). Group 2 countries can potentially supply fishery products to a variety of potential destinations within the RCEP. However, as Teh and Pauly [29] report an extremely high share of low productive small-scale fishers in total catch across the countries of Southeast Asia, a radical growth of fish output will be quite problematic. For example, Thailand has experienced a decline in the value of its fishery exports since 2012, mainly as a result of its eroded competitiveness in small-scale shrimp production [12]. Thailand and Vietnam are competitive in fish trade with many countries worldwide [87], but in the intra-RCEP trade, we record advantages in pairs where the indicative potential is below average, or vice versa, high values of indicative potential are associated with weak advantages or disadvantages. Such discrepancies were also revealed by Hoang [70], who studied correlations between trade and comparative advantages in agricultural trade of Thailand and Vietnam with ASEAN countries.

This reverse relationship between potentials and advantages can be explained by the fact that Group 2 countries have become increasingly dependent on the markets of Group 1 countries [12]. China and to a lesser extent Japan and South Korea absorb additional supply from Southeast Asian traders (even when competitive advantages of the latter are lower) and still have room to expand imports (high indicative trade potential). According to Kurien [126], countries of Southeast Asia have long been producing exports of higher-value fishery products (particularly, crustaceans and mollusks $\left(p_{4}\right)$ ) to more developed countries while at the same time importing lower-priced seafood products for domestic consumption. The FAO [12] forecasts a substantial increase in China's imports of shrimp from Vietnam attributable to a crackdown on illegal and unreported fishing, but we identified neither a distinct advantage nor a significant potential for Vietnam to expand exports of seafood, crustaceans, and mollusks $\left(p_{4}\right)$ to China. Teh et al. $[127,128]$ expect that the catches of demersal fisheries in Vietnam and Thailand must be increased by at least $20 \%$ to account for the unreported industrial catch, but a potential/advantage relationship in VietnamChina and Thailand-China pairs must be checked further when previously unreported data on $p_{4}$ category becomes available in trade statistics.

\subsection{Second-Tier Traders: Australia, Indonesia, Malaysia, New Zealand, Singapore}

Similar to higher-income Group 1 countries, developed economies of Oceania (Australia and New Zealand) and Southeast Asia (Singapore) experience disadvantages in fish trade with Group 2 countries. Along with lower-income Indonesia and Malaysia, Australia and New Zealand are very much oriented on the markets of China, Japan, and South 
Korea [129-131], where they utilize their advantages in $p_{2}$ category at the upper end of the market. Australia, New Zealand, Indonesia, and Malaysia have a reputation for supplying high-quality fish and seafood products to premium-class restaurants across Asia [130,132]. Their advantages in the intraregional fish trade are also driven by the proximity to Singapore and Brunei Darussalam (high potential for an increase in trade in all categories of fishery products) and Philippines (fresh, chilled, and frozen fish $\left(p_{2}\right)$ ). Free trade agreements between Australia and China and New Zealand and China have contributed much to an increase in fish and seafood exports. A rise in supplies of abalone, rock lobsters, and salmonids from Australia and New Zealand to China after the reduction of trade tariffs in 2017 and 2018 demonstrates the flexibility of trade exports to tariffs-a sign that the RCEP agreement could further improve Australia's and New Zealand's positions in the regional fish market.

Indonesia and Malaysia both have a promising prospect to gain from trade in highpriced fishery products with Group 1 countries. Khai et al. [87] revealed a disadvantage of Malaysia in shrimp trade but recorded some extent of competitiveness on non-frozen shrimp products. Alternatively, our study shows that Malaysia can improve its gains from the intra-RCEP trade by expanding exports of crustaceans and mollusks $\left(p_{4}\right)$ to Group 1 and Group 3 countries. Indonesia's advantages (according to Jaya [133], they include marine, brackish water, and freshwater resources by aquaculture and capture fisheries) allow the country to emerge as a supplier of various kinds of fresh fish $\left(p_{2}\right)$ (tilapia is most promising, according to the FAO estimates [12]), crustaceans, and mollusks $\left(p_{4}\right)$ to all four groups of RCEP countries. However, special attention should be paid to quality control and safety of fishery products as the options to sharpen competitive advantage $[134,135]$ (for instance, in light of earlier revealed cases of a high percentage of carcinogenic formaldehyde in salted fish [136] or evidence of COVID-19 transmission through cold supply chains $[10,137,138]$ ).

As regards Singapore, our calculations show untapped advantages in trade in aquaculture products with aquaculture-scarce Brunei Darussalam, Cambodia, and Lao PDR and disadvantages in trade with China, Japan, South Korea, Thailand, and Vietnam, where the aquaculture sector is more developed. The lack of space and stringent environmental regulations [139] challenge the potential of Singapore's aquaculture industry to emerge into a large supplier of fish and seafood products to the regional market. Due to such natural restrictions to large-scale fish production, Singapore primarily relies on an open international trade regime to ensure the food security of its population $[140,141]$ and potentially gains much from joining the RCEP.

\subsection{Small Traders: Brunei Darussalam, Cambodia, Lao PDR, Philippines}

A consensus is yet to be reached about the gains and losses of small economies from the liberalization of fish trade $[28,142,143]$. While for a resource-deficit Singapore, opening up trade is the only option to sustain food supply and achieve food security, for such small fish traders as Group 4 countries, liberalization could mean potential losses to output and trade from more competitive neighboring economies [144]. Echoing earlier estimations of Hoang [70], Bene [28], Sneddon and Fox [145], and Chap et al. [146], we record the weakest advantages in the intra-RCEP fish trade across Group 4 countries. In most cases, high untapped potentials are negated by disadvantages or weak advantages across $p_{2-5}$ categories of fishery products.

Many scholars, including Srean et al. [147], Martin et al. [148], Hartje et al. [149], Joffre et al. [150], and Patricio et al. [151], directly link fisheries with sustained livelihoods, poverty reduction, and food security across the poor rural and coastal communities in Southeast Asia. According to the FAO [12], in Lao PDR and Philippines, people rely heavily on fish for food and protein, while in Cambodia, fish exceeds half of the animal protein intake. This emphasizes a disproportionally important role of fisheries for survival, nutrition, and food security in Group 4 countries. At the same time, as recognized by Bene [28], fishingrelated communities in Asia are amongst the most vulnerable categories of the society due to weak institutional and human capacities to address current challenges to the fishery 
sector. Such external vulnerabilities as climate change [152], declining fish stocks [149], chronic overexploitation of marine resources by extensive fisheries [41,153], and the rise in illegal, unregulated and environmentally unsustainable fishing practices [154], aggravate poverty and food insecurity problems and threaten the entire sustainability of livelihoods. In this sense, further liberalization of fish trade could become an additional source of vulnerability and degradation of competitive advantages for the poor communities who are most dependent on capture fishery.

In the meantime, while losses are often felt immediately, gains from opening up trade for smaller countries could potentially become much larger and more widespread (although they are usually dispersed over time) [144]. Our calculations demonstrate that in some destinations and products, Group 4 countries could match untapped trade potentials with competitive advantages. This well agrees with Kim [155], who found that Brunei Darussalam, Lao PDR, and other small fish traders in Southeast Asia could improve their competitiveness due to export specialties. As Nielsen [16] suggests, the optimal policy in this situation is to remain open to free trade and simultaneously employ fisheries management practices to improve advantages where possible. In general, Group 4 countries can increase their gains from the intra-RCEP trade through increased catches in fishing areas where stocks of certain species are recovering (Cambodia and Philippines), the growth in catches in waters with underfished resources (Brunei Darussalam) and improved utilization of the harvest (Lao PDR) [12]. It can be assumed that an increase in output and exports can substantially enhance the incomes and wellbeing of poor fishers. However, comprehensive research is needed to understand how more intensive fish exports will affect food security in the poor fish-dependent communities. Bene [28] provides an example of Cambodia, one of Asia's top freshwater capture fisheries, where over one-third of rural communities suffer from chronic food shortages. This is just one of many evidences of a complex relationship between the current and potential balance of trade, actual and potential competitive advantages, and food security.

\section{Conclusions}

International trade in fish and seafood products will continue to grow with Asia increasingly gaining importance and becoming the world's largest supplier (about $73 \%$ of the additional exported volumes by 2030, according to the FAO projection [12]). Sustainability of growth in the fish trade, however, can be hampered by the three asymmetries that currently exist between Asian countries: higher dependence on intraregional trade of smaller countries compared to bigger traders, the dominance of higher-income countries in the regional fish market, and income inequality patterns in fish trade that directly affect standards of living, employment, and food security in poorer rural and coastal communities. Given these misbalances, a recent establishment of the RCEP free trade area could further widen the gap between already divergent members of the agreement, increase the outward orientation of fish supplies at the expense of the intraregional trade, degrade competitive advantages of smaller economies, and endanger overall economic and social development of fish-dependent communities.

In an attempt to find solutions to the optimization of the intra-RCEP trade in fish and seafood products, we revealed indicative untapped potentials in bilateral trade between individual RCEP members (210 pairs of countries) and then matched them with comparative, trade, and competitive advantages in the fishery sector (overall trade in fish plus trade in the four categories of fishery products). Having superimposed the advantages scale (strong, average, weak advantages, or a disadvantage) upon the potentials scale (high, above average, below average, or low potentials), we found that in many pairs of countries, the two parameters were mismatched. Specifically, in extreme points, high untapped values of bilateral trade coexisted with disadvantages in one of the countries, while strong advantages were observed in pairs where a potential to increase trade was low. The differentiation of potentials/advantages relationships across higher-income and less-developed RCEP countries allowed us to identify advantageous, disadvantageous, and 
neutral trading destinations and products for each of the fifteen economies. The revealed tendencies and policy implications can be summarized as follows:

- Group 1: major fish traders (China, Japan, and South Korea) have made good use of their potentials and advantages in trade with other countries of the region, as well as between themselves. Still, there are advantages in trade in high-priced fishery products with Group 3 countries (bivalves and fresh and frozen fish $\left(p_{2}\right)$ from China and South Korea to Australia and Singapore and prepared and preserved fishery products $\left(p_{5}\right)$ from Japan to Australia and New Zealand) and Group 4 economies (all four categories of fishery products to Brunei Darussalam, Cambodia, Lao PDR, and Philippines).

- Group 2: Myanmar, Thailand, and Vietnam have the RCEP's highest share of fishery products in trade turnover and enjoy the strongest advantages in trade with higherincome Group 1 countries. Group 2 countries can further gain from the intra-RCEP trade by increasing exports of fresh, chilled, and frozen fish $\left(p_{2}\right)$ and crustaceans, mollusks, and aquatic invertebrates $\left(p_{4}\right)$ all over the region. However, we underline a growing dependence of Group 2 countries on Chinese, Japanese, and Korean markets which could transform into a threat to food security for the former: by exporting higher-value fishery products to more developed economies, Group 2 countries import lower-priced seafood products of lower nutritional value and quality for domestic consumption.

- Group 3: Australia, Indonesia, Malaysia, New Zealand, and Singapore have a low contribution to the intra-RCEP trade in fish and possess weaker advantages in trade with both Group 1 and Group 2 countries. For them, the most promising trading destinations are smaller Group 4 economies, such as Brunei Darussalam, Cambodia, Lao PDR, and Philippines. Also, there is a potential to increase supplies of high-quality fishery products from Australia and New Zealand to Group 1 countries.

- Group 4: the RCEP's smallest traders (Brunei Darussalam, Cambodia, Lao PDR, and Philippines) are the least advantageous countries in the region in terms of fish trade. At the same time, the fishery sector plays a crucial role in supporting livelihood, providing employment, and ensuring food security in the poor rural and coastal communities. For Group 4 countries, we recorded the highest frequency of mismatches between high untapped trade potentials and disadvantages or weak competitive advantages. Still, our findings show an opportunity to increase exports of chilled and frozen fish $\left(p_{2}\right)$ from Philippines and Cambodia to Australia, Singapore, and Thailand, crustaceans and mollusks $\left(p_{4}\right)$ from Brunei Darussalam to Australia, Japan, and South Korea, and fresh fish $\left(p_{2}\right)$ from Lao PDR to other Group 4 countries.

The five-stage approach applied in this paper allowed us to reveal comparative, trade, and competitive advantages in the intra-RCEP trade, match those advantages with existing untapped trade potentials, and identify advantageous and disadvantageous trading destinations and products for individual RCEP countries. The implications of these findings are two-fold, covering methodological and practical issues. The latter adds to the knowledge about fish trade in Asia by detailing the analysis of the country-to-country and the country-to-region trade flows and revealing how each RCEP member can better utilize an individual combination of competitive advantages in order to optimize its gains from the intraregional trade. The methodological implication for further research is that neither simple calculation of indicative trade flows, nor separate studies of any type of advantage, are sufficient to decide whether trade between countries $i$ and $j$ could be gainful for both sides. In bilateral trade, exports and imports flows could be affected by many non-trade contributing factors, including size and level of development of counterparts, distance between them, transportation costs and logistics, customs regulations, currency exchange rates, and a variety of economic, social, and political parameters. In our study, we focused on comparing current trade volumes with advantages, that is, how far a country-to-country trade deviates from a country-to-region trade and how this deviation corresponds with the three types of advantages. Matching between the RCA, the RTA, and the LI values, as well 
as between advantages and indicative trade potentials, might necessarily be undertaken to establish a reliable and realistic picture of potential gains or losses of a particular country in trade with its counterparts. However, the establishment of more comprehensive multifactor models could be helpful for a deeper investigation of the potentials-advantages framework, where diverse effects on the intra-RCEP bilateral trade should be further tested in the extra-area format with the use of estimation models like the gravity equation [58] and consideration of trade costs [156], non-unitary elasticities [157], market volatilities and price expectations of fish traders and stakeholders [158,159], and sector-level variables (tariffs and non-tariff measures on fishery products, support of medium and small-scale fisheries, fleet renewal programs, food safety regulations, etc.) [160-164].

Author Contributions: V.E. and G.T. designed the research framework; V.E. conceptualized the materials and methods; A.I. performed the data collection; V.E., G.T. and A.I. analyzed the data; V.E. wrote the paper. All authors have read and agreed to the published version of the manuscript.

Funding: This research received no external funding.

Institutional Review Board Statement: Not applicable.

Informed Consent Statement: Not applicable.

Data Availability Statement: The data presented in this study are available on request from the corresponding author.

Conflicts of Interest: The authors declare no conflict of interest. 


\section{Appendix A}

Table A1. Degrees of trade potential $\left(R_{i j}\right)$ in trade in fish, crustaceans, and mollusks $\left(p_{1}\right)$ within the RCEP, average in $2000-2019$.

\begin{tabular}{|c|c|c|c|c|c|c|c|c|c|c|c|c|c|c|c|c|}
\hline Countries & AUS & BRN & КНM & $\mathrm{CHN}$ & IDN & JPN & LAO & MYS & MMR & NZL & PHL & SGP & KOR & THA & VNM & RCEP \\
\hline AUS & & 0.011 * & $0.009 *$ & $0.614^{* *}$ & 0.348 * & $0.805^{* *}$ & $0.002 *$ & 0.109 * & $1.736^{* * *}$ & $1.544^{* * *}$ & 0.031 * & $0.069 *$ & 0.014 * & $0.512 *$ & $5.054^{* * *}$ & 0.411 * \\
\hline BRN & $0.432 *$ & & $0.004 *$ & $1.291^{* * *}$ & $0.325 *$ & $0.212 *$ & $0.001 *$ & $15.987^{* * * *}$ & $0.273 *$ & 0.248 * & 0.168 * & $3.961^{* * *}$ & $0.074 *$ & $1.063^{* *}$ & 0.640 ** & $1.541^{* * *}$ \\
\hline KHM & 0.110 * & 0.001 * & & $0.675^{\text {** }}$ & 0.005 * & 0.236 * & 0.004 * & 0.014 * & 0.001 * & 0.029 * & $0.002 *$ & $0.077^{*}$ & $0.128 *$ & $3.718^{* * *}$ & $1.711^{* * *}$ & 0.380 * \\
\hline $\mathrm{CHN}$ & 0.385 * & 0.053 * & $0.202 *$ & & $11.905^{* * * *}$ & $1.202^{* * *}$ & $0.346^{*}$ & $1.965^{* * *}$ & $5.878^{* * *}$ & $2.387^{* * *}$ & $2.263^{* * *}$ & 0.334 * & $2.373^{* * *}$ & $1.066^{* *}$ & $1.899 * * *$ & $1.318^{* * *}$ \\
\hline IDN & $0.046^{*}$ & 0.010 * & $0.001 *$ & $0.249 *$ & & $1.213^{* * *}$ & $0.004 *$ & 0.180 * & $0.169 *$ & $0.015 *$ & $0.068 *$ & $0.182 *$ & 0.037 * & $0.125 *$ & $0.706^{* *}$ & $0.240 *$ \\
\hline JPN & 0.419 * & 0.011 * & $0.078 *$ & $6.321^{* * *}$ & $6.364^{* * * *}$ & & 0.077 * & $0.156^{*}$ & $11.029^{* * * *}$ & $1.363^{* * *}$ & $1.570^{* * *}$ & $0.078 *$ & $1.542^{* * *}$ & $2.124^{* * *}$ & $8.214^{* * * *}$ & $1.920^{* * *}$ \\
\hline LAO & 0.157 * & 0.001 * & 0.415 * & $15.418^{* * * *}$ & $0.433 *$ & $6.527 * * *$ & & 0.037 * & 0.001 * & $1.350^{* * *}$ & $0.001 *$ & 0.109 * & 0.983 ** & $14.498^{* * * *}$ & $13.462 * * * *$ & $7.564^{* * *}$ \\
\hline MYS & $0.168^{*}$ & 0.220 * & $0.005 *$ & $0.634^{* *}$ & $1.293^{* * *}$ & $0.261 *$ & 0.001 * & & $5.353^{* * *}$ & $0.081 *$ & $0.039 *$ & $0.380 *$ & $0.087^{*}$ & $0.395 *$ & $0.663 * *$ & $0.363 *$ \\
\hline MMR & $0.028 *$ & 0.003 * & $0.001 *$ & $0.412 *$ & $0.011 *$ & $0.228 *$ & $0.001 *$ & 0.108 * & & $0.001 *$ & $0.001 *$ & $0.107 *$ & $0.012 *$ & 0.397 * & $0.022 *$ & 0.127 * \\
\hline NZL & $0.460 *$ & 0.011 * & 0.008 * & $0.638^{* * *}$ & 0.015 * & 0.276 * & 0.005 * & 0.018 * & 0.016 * & & 0.024 * & 0.036 * & 0.088 * & 0.107 * & 0.083 * & 0.175 * \\
\hline PHL & 0.015 * & 0.005 * & $0.001 *$ & $0.458 *$ & 0.070 * & $1.830^{* * *}$ & $0.001 *$ & 0.018 * & $0.008 *$ & $0.022 *$ & & 0.086 * & 0.169 * & $0.060 *$ & 0.200 * & $0.195 *$ \\
\hline SGP & 0.304 * & $0.301 *$ & $0.023 *$ & $0.939 * *$ & $0.847^{* *}$ & $0.510 *$ & $0.003 *$ & $1.462 * * *$ & $5.020 * * *$ & $0.167 *$ & $0.132 *$ & & $0.116^{*}$ & $0.566 * *$ & $0.731 * *$ & $0.560 * *$ \\
\hline KOR & 0.010 * & 0.003 * & $0.046 *$ & $1.350^{* * *}$ & 0.393 * & $2.038^{* * *}$ & 0.010 * & $0.058 *$ & $0.585^{* *}$ & 0.743 ** & $0.288 *$ & $0.025 *$ & & 0.281 * & $2.845^{* * *}$ & $0.629^{* *}$ \\
\hline THA & 0.477 * & 0.049 * & $4.263^{* * *}$ & 0.331 * & $1.393^{* * *}$ & $1.683^{* * *}$ & $12.342 * * * *$ & 0.219 * & $11.340^{* * * *}$ & $0.605^{* *}$ & 0.256 * & 0.144 * & $0.133 *$ & & $1.426^{* * *}$ & 0.526 * \\
\hline VNM & $0.194^{*}$ & 0.035 * & $1.659^{* * *}$ & $0.549^{* *}$ & $0.285^{*}$ & $0.804^{* *}$ & 0.201 * & $0.115^{*}$ & $0.572 * *$ & $0.128 *$ & $0.459 *$ & $0.154 *$ & $0.306^{*}$ & 0.174 * & & $0.375 *$ \\
\hline
\end{tabular}

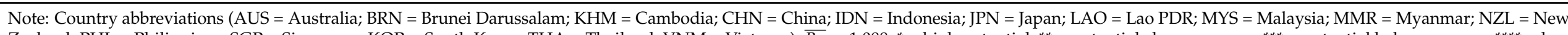

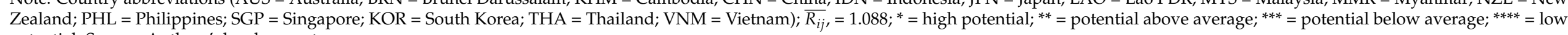
potential. Source: Authors' development. 
Table A2. Degrees of trade potential $\left(R_{i j}\right)$ in trade in fresh, chilled, and frozen fish $\left(p_{2}\right)$ within the RCEP in 2000-2019.

\begin{tabular}{|c|c|c|c|c|c|c|c|c|c|c|c|c|c|c|c|c|}
\hline Countries & AUS & BRN & КНM & $\mathrm{CHN}$ & IDN & JPN & LAO & MYS & MMR & NZL & PHL & SGP & KOR & THA & VNM & RCEP \\
\hline AUS & & $0.005 *$ & $0.013 *$ & $0.202 *$ & 0.227 * & $4.123^{* * *}$ & $0.001 *$ & $0.025 *$ & $1.759^{* * *}$ & $3.707^{* * *}$ & 0.008 * & $0.024 *$ & 0.010 * & 0.061 * & $0.426^{* *}$ & $0.302 *$ \\
\hline BRN & 0.253 * & & $0.011 *$ & $1.488^{* * *}$ & $0.544^{* *}$ & $0.283^{*}$ & $0.001 *$ & $26.079^{* * * *}$ & $0.106^{*}$ & $0.445^{* *}$ & 0.040 * & $5.688^{* * *}$ & $0.019 *$ & $0.465^{* *}$ & $1.488^{* * *}$ & $2.033^{* * *}$ \\
\hline KHM & 0.017 * & $0.002 *$ & & $0.199 *$ & $0.005 *$ & $0.178^{*}$ & $0.024 *$ & $0.022 *$ & $0.001 *$ & $0.001 *$ & $0.001 *$ & $0.157 *$ & $0.015 *$ & $2.422 * * *$ & $1.187^{* * *}$ & $0.221 *$ \\
\hline $\mathrm{CHN}$ & $0.179 *$ & 0.039 * & $0.148 *$ & & $26.435^{* * * *}$ & $1.059 * * *$ & $0.004 *$ & $0.489 * *$ & $3.359^{* * *}$ & $2.920^{* * *}$ & $3.265^{* * *}$ & $0.172 *$ & $2.705^{* * *}$ & $0.357^{* *}$ & $1.285^{* * *}$ & $1.057^{* * *}$ \\
\hline IDN & 0.079 * & $0.013 *$ & $0.003 *$ & 0.321 * & & $0.498^{* *}$ & $0.012 *$ & 0.234 * & 0.111 * & $0.031 *$ & $0.069 *$ & $0.435^{* *}$ & $0.054 *$ & 0.175 * & $0.430 * *$ & 0.234 * \\
\hline JPN & $0.862 * * *$ & 0.014 * & $0.154 *$ & $2.497^{* * *}$ & $2.655^{* * *}$ & & $0.001 *$ & 0.087 * & $1.729 * * *$ & $6.033^{* * *}$ & $0.853 * * *$ & $0.126 *$ & $1.616^{* * *}$ & $0.875^{* * *}$ & $1.773^{* * *}$ & $1.236^{* * *}$ \\
\hline LAO & 0.001 * & 0.001 * & $0.839^{* * *}$ & 0.280 * & $0.224 *$ & $0.007^{*}$ & & 0.017 * & 0.001 * & $0.001 *$ & 0.001 * & $0.040 *$ & $0.226^{*}$ & $12.857^{* * * *}$ & $6.706^{* * *}$ & $2.562^{* * *}$ \\
\hline MYS & $0.051 *$ & 0.131 * & $0.018^{*}$ & $0.802^{* * *}$ & $1.407^{* * *}$ & $0.311 *$ & $0.001 *$ & & $5.070^{* * *}$ & $0.133 *$ & $0.012 *$ & 0.543 ** & 0.035 * & $0.888^{* * *}$ & $0.504 * *$ & $0.467^{* *}$ \\
\hline MMR & 0.030 * & 0.004 * & $0.001 *$ & $0.477^{* *}$ & $0.003 *$ & $0.027^{*}$ & $0.001 *$ & $0.127^{*}$ & & $0.001 *$ & $0.001 *$ & 0.137 * & $0.013^{*}$ & $0.618^{* *}$ & $0.003 *$ & $0.121 *$ \\
\hline NZL & $1.099^{* * *}$ & $0.013 *$ & $0.001 *$ & $0.350 *$ & $0.013 *$ & $0.430 * *$ & $0.001 *$ & $0.012 *$ & $0.009 *$ & & $0.020 *$ & $0.017 *$ & $0.078^{*}$ & $0.037 *$ & $0.041 *$ & 0.170 * \\
\hline PHL & 0.010 * & 0.001 * & $0.001 *$ & $1.030 * * *$ & $0.128 *$ & $2.460 * * *$ & $0.001 *$ & $0.013 *$ & 0.004 * & $0.058 *$ & & $0.062 *$ & $0.150 *$ & 0.108 * & 0.468 ** & $0.302 *$ \\
\hline SGP & 0.105 * & $0.303 *$ & $0.095^{*}$ & $0.390 * *$ & $0.855^{* * *}$ & $0.689 * *$ & $0.002 *$ & $0.772 * * * *$ & $5.063^{* * *}$ & $0.112 *$ & 0.057 * & & $0.133^{*}$ & 0.335 * & $0.647 * *$ & $0.425^{* * *}$ \\
\hline KOR & 0.017 * & $0.001 *$ & $0.014 *$ & $1.849 * * *$ & 0.308 * & $1.965^{* * *}$ & $0.011 *$ & 0.014 * & $0.452 * *$ & $7.597^{* * *}$ & $0.133 *$ & $0.041 *$ & & $0.195 *$ & $1.109^{* * *}$ & 0.690 ** \\
\hline THA & 0.053 * & $0.006^{*}$ & $2.024^{* * *}$ & $0.415^{* *}$ & $1.680^{* * *}$ & $3.064^{* * * *}$ & $8.999 * * *$ & $0.172 *$ & $29.242^{* * * *}$ & 0.362 ** & 0.240 * & $0.102 *$ & $0.325 *$ & & 0.710 ** & $0.562 * *$ \\
\hline VNM & 0.311 * & 0.073 * & $6.005^{* * *}$ & $0.503^{* *}$ & 0.190 * & $0.479 * *$ & 0.285 * & 0.129 * & 0.046 * & 0.146 * & $0.485^{* *}$ & $0.246^{*}$ & $0.187^{*}$ & 0.126 * & & 0.277 * \\
\hline
\end{tabular}

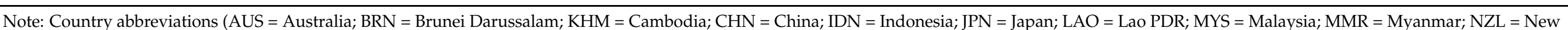

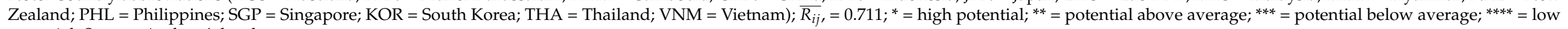
potential. Source: Authors' development.

Table A3. Degrees of trade potential $\left(R_{i j}\right)$ in trade in dried, salted, and smoked fish $\left(p_{3}\right)$ within the RCEP in 2000-2019.

\begin{tabular}{|c|c|c|c|c|c|c|c|c|c|c|c|c|c|c|c|c|}
\hline Countries & AUS & BRN & KHM & CHN & IDN & JPN & LAO & MYS & MMR & NZL & PHL & SGP & KOR & THA & VNM & RCEP \\
\hline AUS & & $0.002 *$ & $0.727 * *$ & 0.514 * & $0.138 *$ & $1.830^{* * *}$ & $0.001 *$ & $0.298 *$ & $0.076^{*}$ & $1.696^{* * *}$ & $4.387^{* * *}$ & $0.432 *$ & $0.309 *$ & $0.324 *$ & $0.394 *$ & $0.502 *$ \\
\hline BRN & $0.826^{* *}$ & & 0.001 * & $1.657^{* * * *}$ & 0.370 * & 0.014 * & 0.001 * & $4.130^{* * *}$ & 0.001 * & 0.333 * & 0.296 * & $12.457^{* * *}$ & 0.044 * & $6.922 * * *$ & $1.072^{* * *}$ & $2.009^{* * *}$ \\
\hline KHM & 0.124 * & $0.001 *$ & & 0.008 * & 0.006 * & $0.002 *$ & 0.001 * & 0.001 * & 0.001 * & 0.001 * & 0.001 * & $0.042 *$ & 0.009 * & $5.865^{* * *}$ & $0.099 *$ & 0.107 * \\
\hline $\mathrm{CHN}$ & $0.036^{*}$ & $0.016 *$ & 0.001 * & & $0.346^{*}$ & $5.812 * * *$ & $0.001 *$ & 0.056 * & $2.074^{* * *}$ & 0.047 * & $3.133^{* * *}$ & 0.221 * & $2.036 * * *$ & $0.683 * *$ & $9.833^{* * *}$ & $1.735^{* * *}$ \\
\hline IDN & 0.024 * & 0.001 * & $0.048 *$ & 0.161 * & & $3.680 * * *$ & 0.001 * & 0.164 * & $1.299 * * *$ & 0.189 * & 0.264 * & 0.244 * & $0.145^{*}$ & 0.613 ** & $2.639 * * *$ & 0.600 ** \\
\hline JPN & 0.361 * & 0.001 * & 0.001 * & $15.052^{* * * *}$ & $2.231^{* * *}$ & & 0.001 * & 0.011 * & $1.990^{* * *}$ & 0.958 ** & $2.437^{* * *}$ & 0.417 * & $1.255^{* * *}$ & $3.446^{* * *}$ & $1.083^{* * *}$ & $2.566^{* * *}$ \\
\hline LAO & $0.001 *$ & 0.001 * & 0.001 * & $10.305^{* * *}$ & $0.002 *$ & $0.001 *$ & & 0.150 * & $0.001 *$ & $0.001 *$ & 0.001 * & $2.962 * * *$ & $4.005^{* * *}$ & $23.813^{* * * *}$ & $27.800^{* * * *}$ & $4.931^{* * *}$ \\
\hline MYS & $0.099 *$ & $0.063 *$ & 0.001 * & 0.100 * & 0.893 ** & $0.039 *$ & $0.001 *$ & & $12.883^{* * *}$ & $0.059 *$ & $3.525^{* * *}$ & $0.551 *$ & $0.012 *$ & $3.094^{* * *}$ & $2.515^{* * *}$ & $0.645^{* *}$ \\
\hline MMR & 0.018 * & 0.001 * & 0.001 * & 0.828 ** & 0.050 * & $0.028 *$ & 0.001 * & $0.349 *$ & & $0.002 *$ & 0.004 * & $0.045 *$ & 0.035 * & 0.813 ** & $0.162 *$ & $0.185^{*}$ \\
\hline NZL & $0.754^{* *}$ & 0.005 * & 0.001 * & 0.063 * & 0.034 * & 0.490 * & 0.001 * & 0.027 * & $0.009 *$ & & $1.652^{* * *}$ & 0.182 * & 0.122 * & 0.018 * & 0.024 * & 0.162 * \\
\hline SGP & 0.094 * & 0.238 * & $0.205 *$ & 0.743 ** & $1.078^{* * *}$ & 0.254 * & 0.030 * & $0.642 * *$ & $4.198^{* * *}$ & $0.595^{* *}$ & $2.025^{* * *}$ & & $0.081 *$ & $0.599 * *$ & $3.265^{* * *}$ & 0.581 ** \\
\hline KOR & 0.106 * & 0.001 * & 0.050 * & $2.442^{* * *}$ & 0.365 * & $1.940 * * *$ & 0.024 * & 0.006 * & 0.024 * & 0.443 * & $7.719^{* * *}$ & $0.092 *$ & & 0.178 * & $4.210^{* * *}$ & $0.737 * *$ \\
\hline THA & $0.103 *$ & $0.008 *$ & $19.065^{* * * *}$ & $0.677^{* *}$ & $0.523 *$ & $0.351 *$ & $3.584 * * *$ & $0.869 * *$ & $2.447^{* * *}$ & $0.044 *$ & $0.850 * *$ & $0.064 *$ & 0.047 * & & $2.696^{* * *}$ & $0.338 *$ \\
\hline VNM & 0.089 * & 0.002 * & $0.962 * *$ & $12.246^{* * *}$ & 0.224 * & 0.321 * & 0.146 * & 0.180 * & $1.959 * * *$ & 0.031 * & 0.105 * & 0.208 * & $0.776^{* *}$ & $0.734^{* *}$ & & 0.517 * \\
\hline
\end{tabular}

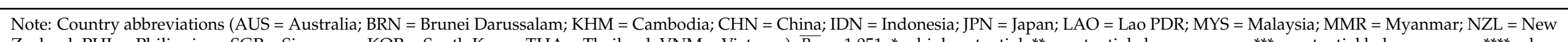

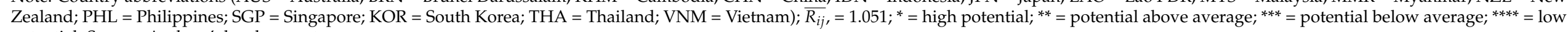
potential. Source: Authors' development. 
Table A4. Degrees of trade potential $\left(R_{i j}\right)$ in trade in crustaceans, mollusks, and aquatic invertebrates $\left(p_{4}\right)$ within the RCEP in $2000-2019$.

\begin{tabular}{|c|c|c|c|c|c|c|c|c|c|c|c|c|c|c|c|c|}
\hline Countries & AUS & BRN & КНМ & $\mathrm{CHN}$ & IDN & JPN & LAO & MYS & MMR & NZL & PHL & SGP & KOR & THA & VNM & RCEP \\
\hline AUS & & $0.035 *$ & $0.008 *$ & 0.602 ** & $1.015^{* * *}$ & $0.518^{* *}$ & $0.032 *$ & $0.152 *$ & $3.736^{* * *}$ & $0.795^{* * *}$ & $0.011 *$ & $0.105 *$ & $0.005 *$ & $0.416^{* *}$ & $3.029^{* * *}$ & 0.453 ** \\
\hline BRN & $0.581 * *$ & & $0.001 *$ & $0.833^{* * *}$ & 0.094 * & $0.126^{*}$ & 0.001 * & $6.235^{* * *}$ & 0.393 ** & $0.053^{*}$ & $0.002 *$ & $1.768^{* * *}$ & $0.036^{*}$ & $0.112 *$ & $0.100 *$ & 0.718 ** \\
\hline KHM & $0.009 *$ & $0.001 *$ & & $0.822 * * *$ & $0.003 *$ & $0.088^{*}$ & 0.001 * & $0.002 *$ & $0.001 *$ & $0.009 *$ & $0.003 *$ & $0.024 *$ & $0.302 *$ & $1.326^{* * *}$ & $0.917^{* * *}$ & 0.234 * \\
\hline $\mathrm{CHN}$ & 0.527 ** & 0.088 * & $0.318 *$ & & $4.117^{* * *}$ & $0.688^{* *}$ & 0.001 * & $3.720^{* * *}$ & $9.745^{* * * *}$ & $3.430^{* * *}$ & $1.569^{* * *}$ & $0.240 *$ & $1.411^{* * *}$ & $2.892 * * *$ & $1.138^{* * *}$ & $1.178^{* * *}$ \\
\hline IDN & $0.041 *$ & $0.009 *$ & $0.002 *$ & 0.229 * & & $2.082^{* * *}$ & 0.035 * & 0.110 * & $0.373 * *$ & $0.012 *$ & $0.048 *$ & $0.113 *$ & $0.025^{*}$ & $0.074^{*}$ & $1.958^{* * *}$ & 0.305 * \\
\hline JPN & $0.564 * *$ & 0.018 * & $0.066^{*}$ & $7.300 * * *$ & $9.266^{* * * *}$ & & $0.002 *$ & 0.317 * & $6.537^{* * *}$ & $0.540 * *$ & $2.935^{* * *}$ & $0.032 *$ & $1.177^{* * *}$ & $2.580 * * *$ & $6.257^{* * *}$ & $2.339^{* * *}$ \\
\hline LAO & 0.728 ** & 0.001 * & $0.001 *$ & 0.253 * & $1.544^{* * *}$ & 0.027 * & & $0.129 *$ & $0.001 *$ & $6.468^{* * *}$ & $0.001 *$ & $0.088 *$ & $0.510^{* *}$ & $7.513 * * *$ & $2.398^{* * *}$ & $2.282 * * *$ \\
\hline MYS & $0.126^{*}$ & $0.155^{*}$ & $0.002 *$ & $0.662 * *$ & $2.120^{* * *}$ & $0.344 *$ & $0.006 *$ & & $13.747^{* * * *}$ & $0.082 *$ & $0.060 *$ & $0.322 *$ & $0.141 *$ & $0.182 *$ & $1.564^{* * *}$ & $0.349 *$ \\
\hline MMR & $0.029 *$ & $0.005 *$ & $0.001 *$ & $0.265^{*}$ & $0.061 *$ & $0.669^{* * *}$ & 0.001 * & $0.073 *$ & & $0.002 *$ & $0.001 *$ & $0.090 *$ & $0.008^{*}$ & $0.216^{*}$ & $0.060 *$ & $0.142 *$ \\
\hline NZL & $0.223 *$ & $0.008 *$ & $0.005 *$ & $1.490^{* * *}$ & 0.034 * & $0.152 *$ & $0.092 *$ & $0.022 *$ & $0.044 *$ & & 0.018 * & $0.022 *$ & $0.117^{*}$ & $0.084 *$ & $0.127 *$ & $0.186^{*}$ \\
\hline PHL & $0.001 *$ & 0.001 * & $0.001 *$ & $0.269 *$ & $0.065 *$ & $1.507^{* * *}$ & 0.001 * & 0.010 * & 0.019 * & $0.010 *$ & & $0.071 *$ & $0.252 *$ & 0.013 * & $0.142 *$ & $0.169 *$ \\
\hline SGP & $0.580 * *$ & $0.267^{*}$ & $0.019 *$ & $1.320 * * *$ & $1.759^{* * *}$ & $0.485^{* *}$ & 0.003 * & $3.809^{* * *}$ & $14.516^{* * * *}$ & $0.137^{*}$ & 0.308 * & & $0.120 *$ & $0.600 * *$ & $1.189 * * *$ & $0.884^{* * *}$ \\
\hline KOR & $0.006 *$ & $0.005 *$ & $0.209 *$ & $2.259 * * *$ & $1.003 * * *$ & $1.937^{* * *}$ & 0.015 * & 0.143 * & $1.707^{* * *}$ & $0.594 * *$ & 0.590 ** & $0.018 *$ & & $0.546 * *$ & $4.448 * * *$ & $0.864^{* * *}$ \\
\hline THA & $0.226 *$ & 0.010 * & $2.002^{* * *}$ & $0.252 *$ & $0.914^{* * *}$ & $1.652 * * *$ & $9.901^{* * * * *}$ & 0.113 * & $8.471^{* * * *}$ & $0.225 *$ & 0.064 * & $0.126 *$ & $0.125 *$ & & $4.758^{* * *}$ & $0.421^{* *}$ \\
\hline VNM & $0.192 *$ & 0.004 * & $1.885^{* * *}$ & $0.768^{* * *}$ & $1.401^{* * *}$ & $0.937^{* * *}$ & $0.172 *$ & 0.114 * & $2.591 * * *$ & $0.188^{*}$ & $0.464^{* *}$ & $0.171^{*}$ & $0.477^{* *}$ & $0.378^{* *}$ & & 0.559 ** \\
\hline
\end{tabular}

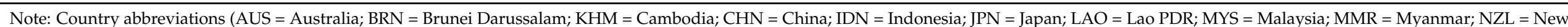

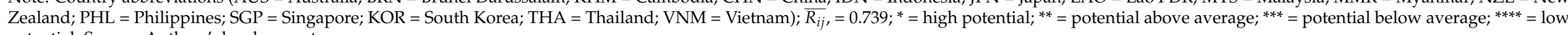
potential. Source: Authors' development.

Table A5. Degrees of trade potential $\left(R_{i j}\right)$ in trade in prepared and preserved fish and aquatic invertebrates $\left(p_{5}\right)$ within the RCEP in $2000-2019$.

\begin{tabular}{|c|c|c|c|c|c|c|c|c|c|c|c|c|c|c|c|c|}
\hline Countries & AUS & BRN & KHM & CHN & IDN & JPN & LAO & MYS & MMR & NZL & PHL & SGP & KOR & THA & VNM & RCEP \\
\hline AUS & & $0.001 *$ & $0.004 *$ & $2.037^{* * *}$ & 0.477 * & 0.129 * & $0.001 *$ & $0.401 *$ & $0.881^{* *}$ & $0.689 *$ & $0.452 *$ & 0.170 * & $0.039 *$ & $2.128^{* * *}$ & $4.882^{* * *}$ & $0.804^{* *}$ \\
\hline BRN & $0.370 *$ & & 0.001 * & $6.316^{* * *}$ & $2.371^{* * *}$ & $1.035^{* *}$ & 0.001 * & $11.624^{* * *}$ & $0.041 *$ & $1.716^{* * *}$ & $5.052^{* * *}$ & $23.316^{* * * *}$ & $1.494^{* * *}$ & $25.890^{* * * * *}$ & 1.135 ** & $5.718^{* * *}$ \\
\hline KHM & $0.076^{*}$ & 0.001 * & & $3.283^{* * *}$ & 0.013 * & $1.381^{* *}$ & 0.001 * & 0.058 * & $0.001 *$ & 0.272 * & 0.004 * & 0.124 * & 0.157 * & $21.141^{* * * *}$ & $15.099^{* * * *}$ & $2.131^{* * *}$ \\
\hline $\mathrm{CHN}$ & $0.429 *$ & $0.052 *$ & 0.181 * & & $11.573^{* * *}$ & $2.178^{* * *}$ & $0.513 *$ & $2.281^{* * *}$ & $2.039 * * *$ & $1.249 * *$ & $1.487^{* * *}$ & $0.709 *$ & $4.746^{* * *}$ & $22.828^{* * * *}$ & $1.921^{* * *}$ & $2.123^{* * *}$ \\
\hline JPN & 0.296 * & 0.001 * & 0.063 * & $4.562^{* * *}$ & $6.713^{* * *}$ & & 0.104 * & 0.106 * & $1.188 * *$ & 0.156 * & $9.392 * * *$ & $0.266^{*}$ & $2.128^{* * *}$ & $9.827^{* * * *}$ & $6.499 * * *$ & $2.950^{* * *}$ \\
\hline LAO & 0.016 * & 0.001 * & 0.009 * & $8.437^{* * *}$ & 0.001 * & $22.280^{* * * *}$ & & 0.001 * & 0.001 * & 0.001 * & 0.001 * & 0.165 * & $6.692^{* * *}$ & $15.970^{* * * *}$ & $5.681^{* * *}$ & $4.232^{* * *}$ \\
\hline MYS & 0.529 * & $0.664 *$ & 0.003 * & $0.441 *$ & $0.483 *$ & $0.060 *$ & 0.001 * & & $0.752 * *$ & $0.046^{*}$ & $0.468 *$ & 0.549 * & $0.032 *$ & 0.395 * & $1.033 * *$ & 0.297 * \\
\hline MMR & 0.019 * & 0.001 * & 0.001 * & $1.381^{* *}$ & 0.003 * & 0.037 * & 0.001 * & $0.022 *$ & & $0.002 *$ & 0.004 * & 0.027 * & $0.015 *$ & 0.654 * & 0.013 * & 0.106 * \\
\hline NZL & 0.527 * & 0.012 * & 0.011 * & 0.854 ** & 0.009 * & 0.065 * & 0.001 * & 0.033 * & 0.011 * & & 0.048 * & 0.155 * & 0.072 * & 0.393 * & 0.352 * & 0.237 * \\
\hline SGP & 0.598 * & 0.354 * & 0.006 * & $1.768^{* * *}$ & 0.844 ** & 0.334 * & $0.002 *$ & $2.148^{* * *}$ & 0.311 * & $0.360 *$ & $1.131^{* *}$ & & $0.091 *$ & 1.049 ** & $4.157^{* * *}$ & $0.831^{* *}$ \\
\hline KOR & 0.023 * & 0.008 * & 0.009 * & $1.249 * *$ & 0.352 * & $2.772 * * *$ & 0.009 * & $0.046^{*}$ & $0.224 *$ & 0.198 * & $2.088^{* * *}$ & 0.018 * & & 0.377 * & $13.277^{* * *}$ & 0.584 * \\
\hline THA & $1.319^{* *}$ & 0.225 * & $10.005^{* * * *}$ & 0.602 * & $0.856^{* *}$ & 1.379 ** & $14.550^{* * * *}$ & 0.684 * & $1.650^{* * *}$ & $1.642^{* * *}$ & $3.850 * * *$ & 0.216 * & $0.156^{*}$ & & $7.778^{* * *}$ & $0.909^{* *}$ \\
\hline VNM & $0.142 *$ & $0.001 *$ & $1.094^{* *}$ & 0.087 * & $0.020 *$ & $1.236^{* *}$ & 0.188 * & 0.053 * & $0.093 *$ & $0.078 *$ & 0.091 * & 0.057 * & 0.447 * & $0.294 *$ & & 0.334 * \\
\hline
\end{tabular}

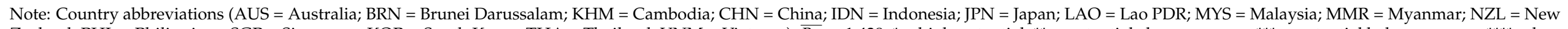

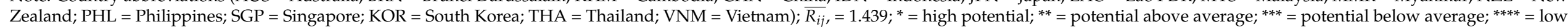
potential. Source: Authors' development. 


\section{Appendix B}

Table A6. RCA, RTA, and LI values for total trade in fish, crustaceans, and mollusks $\left(p_{1}\right)$ in RCEP countries, average in 2000-2019.

\begin{tabular}{|c|c|c|c|c|c|c|c|c|c|c|c|c|c|c|c|c|}
\hline Countries & AUS & BRN & КHM & $\mathrm{CHN}$ & IDN & JPN & LAO & MYS & MMR & NZL & PHL & SGP & KOR & THA & VNM & RCEP \\
\hline AUS RCA & & 0.266 & 0.079 & 0.255 & 0.139 & 0.769 & 0.048 & 0.211 & 0.069 & 0.137 & 0.050 & 0.633 & 0.013 & 0.232 & $7.513 *$ & 0.475 \\
\hline AUS RTA & & $0.222 * *$ & -0.287 & -0.300 & -1.043 & $0.656^{* *}$ & 0.048 ** & -0.719 & -60.380 & -3.317 & -0.690 & $0.552 * *$ & -0.088 & -4.120 & $2.419 * *$ & -0.642 \\
\hline AUS LI & & -0.006 & -0.008 & $1.734^{* * *}$ & -1.793 & $19.942^{* * *}$ & $0.001^{* * *}$ & -2.301 & -0.565 & -8.080 & -0.142 & $2.369^{* * *}$ & -0.187 & -14.847 & $3.884^{* * *}$ & -0.035 \\
\hline BRN RCA & 0.045 & & 0.001 & 0.180 & 0.005 & 0.004 & 0.001 & 0.184 & 0.001 & 0.001 & 0.013 & 0.037 & 0.002 & 0.022 & 0.034 & 0.022 \\
\hline BRN RTA & -0.611 & & -0.347 & -0.107 & -0.823 & -0.121 & $0.001 * *$ & -4.204 & -75.809 & -6.337 & -3.721 & -1.320 & -0.086 & -1.558 & -13.116 & -1.816 \\
\hline BRN LI & $1.773 * * *$ & & -0.001 & $2.681^{* * *}$ & $0.011^{* * *}$ & $0.657^{* * * *}$ & $0.001^{* * *}$ & -3.680 & -0.135 & -0.109 & -0.061 & -1.244 & $0.132 * * *$ & $0.087^{* * *}$ & -0.110 & -0.954 \\
\hline KHM RCA & $1.437 *$ & 0.219 & & 0.473 & 0.005 & 0.121 & $1.016 *$ & 0.086 & 0.001 & 0.001 & 0.017 & 0.121 & 0.850 & $1.132 *$ & 0.163 & 0.406 \\
\hline KHM RTA & $1.355^{* *}$ & $0.219 * *$ & & $0.339 * *$ & -0.009 & -0.436 & $1.016^{* *}$ & $0.061 * *$ & -0.029 & -7.637 & -0.095 & $0.096^{* *}$ & 0.806 ** & $0.470^{* *}$ & -0.766 & -0.010 \\
\hline KNM LI & $3.130 * * *$ & $0.022 * * *$ & & $3.118^{* * *}$ & -0.036 & $0.900^{* * *}$ & $0.101^{* * *}$ & $0.211 * * *$ & $0.001^{* * *}$ & -0.250 & -0.006 & $1.356^{* * *}$ & $3.019^{* * *}$ & -0.818 & -10.747 & -0.131 \\
\hline CHN RCA & 0.622 & 0.157 & 0.102 & & 0.485 & $2.686^{*}$ & 0.169 & $1.305 *$ & 0.011 & 0.797 & $1.585 *$ & 0.419 & $1.779 *$ & $1.732 *$ & 0.258 & $1.546^{*}$ \\
\hline CHN RTA & $0.406^{* *}$ & -0.094 & -0.486 & & -0.523 & $2.497^{* *}$ & $0.168 * *$ & $1.205^{* *}$ & -1.409 & -4.174 & $1.419^{* *}$ & $0.379 * *$ & $1.668 * *$ & $1.155^{* *}$ & -0.426 & $1.259 * *$ \\
\hline CHN LI & -1.616 & -0.010 & -0.032 & & -3.275 & $9.018^{* * *}$ & $0.006^{* * *}$ & $0.816^{* * *}$ & -0.743 & -4.650 & 0.636 & $0.453^{* * *}$ & $2.862 * * *$ & -1.439 & -2.027 & $1.367^{* * *}$ \\
\hline IDN RCA & $1.002 *$ & 0.385 & 0.009 & 1.107 * & & $3.101 *$ & 0.354 & $1.008^{*}$ & 0.031 & 0.265 & 0.199 & 0.666 & 0.468 & $2.143 *$ & $3.640 *$ & 1.578 * \\
\hline IDN RTA & $0.873 * *$ & 0.380 ** & $0.004 * *$ & $0.688^{* *}$ & & 2.986 ** & $0.354 * *$ & $0.695^{* *}$ & -1.667 & $0.096^{* *}$ & -0.221 & $0.604 * *$ & $0.417^{* *}$ & $2.028^{* *}$ & $3.308^{* *}$ & $1.381 * *$ \\
\hline IDN LI & -0.059 & $0.001^{* * *}$ & $0.001^{* * *}$ & -7.391 & & $8.093^{* * *}$ & $0.001^{* * *}$ & -1.143 & -0.111 & -0.089 & -0.166 & -0.295 & $0.198^{* * *}$ & $0.510^{* * *}$ & $0.451^{* * *}$ & $0.589^{* * *}$ \\
\hline JPN RCA & 0.081 & 0.106 & 0.539 & 0.222 & 0.089 & & 0.461 & 0.090 & 0.014 & 0.730 & 0.128 & 0.121 & 0.300 & 0.463 & 0.938 & 0.255 \\
\hline JPN RTA & -0.858 & $0.098^{* *}$ & $0.396^{* *}$ & -2.102 & -3.909 & & $0.461 * *$ & -0.332 & -13.809 & -5.005 & -2.247 & -0.134 & -2.715 & -6.437 & -8.200 & -2.477 \\
\hline JPN LI & -0.423 & $0.003^{* * *}$ & $0.026^{* * *}$ & -0.750 & -1.694 & & $0.008^{* * *}$ & $0.143^{* * *}$ & -0.161 & $0.075^{* * *}$ & -0.108 & $0.606^{* * *}$ & $1.950 * * *$ & $0.371^{* * *}$ & -0.044 & -1.004 \\
\hline LAO RCA & 0.001 & 0.001 & 0.001 & 0.001 & 0.001 & 0.001 & & 0.006 & 0.001 & 0.001 & 0.001 & 0.001 & 0.073 & 0.003 & 0.002 & 0.003 \\
\hline LAO RTA & -0.071 & 0.001 ** & -1.409 & -0.168 & -0.658 & -0.788 & & -0.017 & 0.001 ** & -13.476 & 0.001 ** & -0.018 & $0.018^{* *}$ & -0.226 & -0.351 & -0.232 \\
\hline LAO LI & -0.001 & $0.004^{* * *}$ & -0.005 & -0.134 & -0.005 & -0.133 & & $0.017^{* * *}$ & $0.004 * * *$ & -0.024 & 0.004 & $0.002 * * *$ & $0.494^{* * *}$ & -0.157 & -0.067 & -2.593 \\
\hline MYS RTA & $0.521 * *$ & 0.897 ** & -0.075 & -0.692 & -1.836 & $0.229 * *$ & $0.001 * *$ & & -21.596 & -1.088 & -0.048 & $0.310^{* *}$ & $0.339 * *$ & -1.337 & -1.028 & -0.350 \\
\hline MYS LI & $5.573 * * *$ & $0.632 * * *$ & $0.001^{* * *}$ & -9.846 & -8.487 & $6.587^{* * *}$ & $0.001^{* * *}$ & & -2.672 & -0.305 & 0.087 & $11.729^{* * *}$ & $2.988^{* * *}$ & -6.825 & $0.537^{* * *}$ & -0.082 \\
\hline MMR RCA & $48.069 *$ & $18.029 *$ & 0.001 & $4.324 *$ & $2.160 *$ & $11.568 *$ & 0.001 & 14.540 * & & $5.629 *$ & 0.222 & $8.312 *$ & $1.837 *$ & $3.477^{*}$ & $5.800 *$ & $5.199 *$ \\
\hline MMR RTA & $48.027^{* *}$ & $18.029 * *$ & $0.001 * *$ & $4.256^{* *}$ & $2.102 * *$ & $11.542 * *$ & $0.001 * *$ & 14.524 ** & & $5.070 * *$ & 0.220 ** & 8.294 ** & $1.833 * *$ & $3.054 * *$ & $5.763^{* *}$ & $5.104 * *$ \\
\hline MMR LI & $0.088^{* * *}$ & $0.001^{* * *}$ & $0.001^{* * *}$ & $0.100 * * *$ & -0.078 & $0.603^{* * *}$ & $0.001^{* * *}$ & $0.324^{* * *}$ & & -0.035 & $0.001^{* * *}$ & $0.189 * * *$ & $0.036^{* * *}$ & -1.245 & $0.017^{* * *}$ & $3.037^{* * *}$ \\
\hline NZL RCA & $2.704 *$ & $4.757^{*}$ & $5.828 *$ & $4.092 *$ & 0.137 & $4.232 *$ & $2.864 *$ & $1.107^{*}$ & 0.273 & & 0.557 & $2.955 *$ & $1.981 *$ & $3.429 *$ & $1.464 *$ & $3.069 *$ \\
\hline NZL RTA & $2.521 * *$ & $4.756^{* *}$ & $5.828^{* *}$ & $3.425 * *$ & -0.170 & $3.763^{* *}$ & $2.864 * *$ & $0.799 * *$ & -4.164 & & -0.454 & 2.904 ** & -0.439 & -1.063 & -4.174 & $2.298^{* *}$ \\
\hline NZL LI & $6.753 * * *$ & $0.019 * * *$ & $0.011^{* * *}$ & $4.937 * * *$ & -0.261 & $3.675^{* * *}$ & $0.001 * * *$ & -0.276 & -0.012 & & -0.025 & $0.864^{* * *}$ & -4.494 & -8.634 & -2.558 & $1.480^{* * *}$ \\
\hline PHL RCA & 0.558 & $2.069 *$ & 0.068 & 0.365 & 0.351 & $1.288 *$ & 0.001 & 0.124 & 0.047 & 0.988 & & 0.330 & 0.872 & 0.452 & 0.815 & 0.734 \\
\hline PHL RTA & $0.521 * *$ & $2.061 * *$ & $0.051^{* *}$ & -0.493 & $0.038 * *$ & $1.072 * *$ & $0.001 * *$ & $0.065^{* *}$ & -0.187 & $0.350 * *$ & & $0.256 * *$ & $0.639 * *$ & $0.375^{* *}$ & -2.073 & $0.315^{* *}$ \\
\hline PHL LI & $0.537^{* * *}$ & $0.035^{* * *}$ & $0.002^{* * *}$ & -16.625 & -2.160 & $24.538^{* * *}$ & $0.001 * * *$ & $0.073^{* * *}$ & -0.012 & -0.645 & & $2.097^{* * *}$ & $1.631^{* * *}$ & $1.315^{* * *}$ & -10.787 & $0.281^{* * *}$ \\
\hline SGP RCA & 0.054 & 0.774 & 0.019 & 0.031 & 0.050 & 0.171 & 0.027 & 0.109 & 0.026 & 0.040 & 0.075 & & 0.058 & 0.075 & 0.200 & 0.083 \\
\hline
\end{tabular}


Table A6. Cont.

\begin{tabular}{|c|c|c|c|c|c|c|c|c|c|c|c|c|c|c|c|c|}
\hline Countries & AUS & BRN & КHM & $\mathrm{CHN}$ & IDN & JPN & LAO & MYS & MMR & NZL & PHL & SGP & KOR & THA & VNM & RCEP \\
\hline SGP RTA & -0.808 & $0.727^{* *}$ & -0.103 & -0.354 & -0.738 & -0.011 & $0.027^{* *}$ & -0.299 & -9.499 & -3.654 & -0.217 & & -0.010 & -0.789 & -3.429 & -0.416 \\
\hline SGP LI & -0.371 & $1.526^{* * *}$ & $0.035^{* * *}$ & -3.400 & -1.842 & $3.584^{* * *}$ & $0.003^{* * *}$ & $2.747^{* * *}$ & -1.315 & -0.875 & 0.504 & & $1.141^{* * *}$ & -1.580 & -0.157 & -0.324 \\
\hline KOR RCA & 0.081 & 0.055 & 0.043 & 0.198 & 0.054 & 2.406 * & 0.042 & 0.069 & 0.017 & $4.006^{*}$ & 0.172 & 0.059 & & $1.641 *$ & 0.217 & 0.552 \\
\hline KOR RTA & $0.058^{* *}$ & $0.051 * *$ & -0.992 & -1.620 & -0.521 & $2.029^{* *}$ & -0.034 & -0.332 & -1.519 & $2.162 * *$ & -0.787 & -0.045 & & -2.122 & -7.556 & -0.698 \\
\hline KOR LI & $0.179^{* * *}$ & $0.002 * * *$ & -0.016 & -17.179 & -1.050 & $25.013^{* * *}$ & $0.001^{* * *}$ & -0.517 & -0.083 & $1.325^{* * *}$ & -0.123 & $0.181^{* * *}$ & & $0.891^{* * *}$ & -8.624 & -0.774 \\
\hline THA RCA & $3.999 *$ & $1.265 *$ & 0.594 & 0.713 & 0.082 & $6.291 *$ & 0.186 & 0.826 & 0.503 & $3.745^{*}$ & 0.110 & 0.775 & $3.387 *$ & & $1.062 *$ & $2.198 *$ \\
\hline THA RTA & $3.753 * *$ & $1.247^{* *}$ & -0.871 & -0.174 & -4.023 & $5.701 * *$ & $0.183^{* *}$ & $0.438^{* *}$ & -2.273 & -0.797 & -1.361 & $0.716^{* *}$ & $1.404^{* *}$ & & -4.814 & $1.101^{* *}$ \\
\hline THA LI & $5.231^{* * *}$ & $0.024^{* * *}$ & $0.160 * * *$ & -6.225 & -8.260 & $18.165^{* * *}$ & $0.092 * * *$ & $0.066^{* * *}$ & -2.531 & -0.173 & -1.234 & $1.303^{* * *}$ & -2.518 & & -4.100 & $1.626^{* * *}$ \\
\hline VNM RCA & $4.480 *$ & $6.424 *$ & 0.788 & 2.796 * & 0.297 & $9.211 *$ & 0.272 & $2.054 *$ & 0.094 & $5.225 *$ & $2.295 *$ & $3.110 *$ & $6.881 *$ & $5.257 *$ & & $4.756 *$ \\
\hline VNM RTA & $4.129 * *$ & $6.402 * *$ & $0.639 * *$ & $2.621 * *$ & -1.573 & 8.620 ** & $0.272 * *$ & $1.819^{* *}$ & -4.412 & $3.787^{* *}$ & $1.460 * *$ & $2.969 * *$ & $6.688^{* *}$ & $4.899 * *$ & & $4.442 * *$ \\
\hline VNM LI & $0.5648^{* * *}$ & $0.005^{* * *}$ & $0.059 * * *$ & -0.529 & -2.473 & $2.797 * * *$ & $0.006^{* * *}$ & -0.140 & -0.241 & -0.212 & -0.084 & $0.022 * * *$ & $0.706^{* * *}$ & -0.480 & & $3.093^{* * *}$ \\
\hline
\end{tabular}

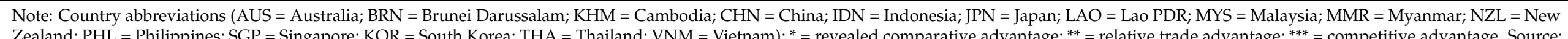

Authors' development.

Table A7. RCA, RTA, and LI values for fresh, chilled, and frozen fish $\left(p_{2}\right)$ in RCEP countries, average in 2000-2019.

\begin{tabular}{|c|c|c|c|c|c|c|c|c|c|c|c|c|c|c|c|c|}
\hline Countries & AUS & BRN & KHM & CHN & IDN & JPN & LAO & MYS & MMR & NZL & PHL & SGP & KOR & THA & VNM & RCEP \\
\hline AUS RCA & & 0.565 & 0.001 & 0.102 & 0.298 & 1.288 * & 0.001 & 0.075 & 0.137 & 0.092 & 0.102 & 0.140 & 0.027 & 0.272 & 0.528 & 0.428 \\
\hline AUS RTA & & $0.563 * *$ & -0.264 & -0.057 & -0.885 & $1.262 * *$ & $0.001 * *$ & -0.096 & -71.917 & -6.223 & -0.139 & 0.068 ** & $0.007 * *$ & -0.040 & -4.230 & -0.293 \\
\hline AUS LI & & $0.017^{* * *}$ & -0.011 & $0.782^{* * *}$ & -2.514 & $39.766^{* * *}$ & $0.001 * * *$ & -0.610 & -1.084 & -25.558 & $0.021 * * *$ & $0.237^{* * *}$ & $0.254^{* * *}$ & -0.819 & -10.483 & -0.488 \\
\hline BRN RCA & 0.001 & & 0.001 & 0.194 & 0.013 & 0.002 & 0.001 & 0.289 & 0.001 & 0.002 & 0.004 & 0.013 & 0.001 & 0.055 & 0.056 & 0.023 \\
\hline BRN RTA & -1.070 & & -0.946 & -0.129 & -1.258 & -0.234 & $0.001^{* *}$ & -5.506 & -29.054 & -11.115 & -0.862 & -2.010 & -0.029 & -0.355 & -29.901 & -2.396 \\
\hline BRN LI & -0.071 & & -0.002 & $2.239 * * *$ & $0.206^{* * *}$ & $0.229^{* * *}$ & $0.001^{* * *}$ & -1.878 & -0.029 & -0.100 & -0.007 & -1.430 & -0.003 & $1.003 * * *$ & -0.160 & -1.492 \\
\hline KHM RCA & 0.242 & 0.614 & & 0.293 & 0.015 & 0.202 & $2.830 *$ & 0.212 & 0.001 & 0.001 & 0.047 & 0.294 & 0.103 & 1.554 * & 0.020 & 0.404 \\
\hline KHM RTA & $0.234^{* *}$ & 0.613 ** & & 0.272 ** & 0.001 ** & $0.046^{\text {** }}$ & $2.828^{* *}$ & $0.204^{* *}$ & -0.078 & 0.001 ** & $0.009 * *$ & $0.275^{* *}$ & $0.096^{* *}$ & $1.397^{* * *}$ & -0.667 & $0.223^{* *}$ \\
\hline KNM LI & $0.750 * * *$ & $0.085^{* * *}$ & & $3.888^{* * *}$ & -0.121 & $4.074^{* * *}$ & $0.401 * * *$ & $0.869^{* * *}$ & -0.002 & $0.001^{* * *}$ & $0.019^{* * *}$ & $4.908^{* * *}$ & $0.465^{* * *}$ & $15.697 * * *$ & -31.034 & $0.401^{* * *}$ \\
\hline CHN RCA & 0.146 & 0.148 & 0.023 & & 0.983 & $2.286 *$ & 0.001 & 0.887 & 0.008 & 0.374 & 2.714 * & 0.213 & $2.212 *$ & $1.718^{*}$ & 0.322 & 1.500 * \\
\hline CHN RTA & $0.028^{* *}$ & -0.131 & -0.261 & & 0.051 ** & $2.004^{* *}$ & -0.001 & $0.799 * *$ & -0.991 & -4.816 & $2.522 * *$ & $0.169 * *$ & $2.076^{* *}$ & 1.279 ** & -0.855 & $1.183^{* *}$ \\
\hline CHN LI & -1.102 & -0.011 & -0.018 & & -2.335 & $6.065^{* * *}$ & $0.001 * * *$ & $0.551^{* * *}$ & -0.526 & -4.940 & $1.651^{* * *}$ & $0.168^{* * *}$ & $4.568^{* * *}$ & -0.444 & -3.631 & $1.231^{* * *}$ \\
\hline IDN RCA & 0.987 & 0.637 & 0.010 & $1.285^{*}$ & & $1.879 *$ & 0.478 & $1.909 *$ & 0.064 & 0.247 & 0.472 & $1.305^{*}$ & 0.685 & $4.344 *$ & $4.672 *$ & 1.635 * \\
\hline IDN RTA & $0.717^{* *}$ & $0.623^{* *}$ & -0.005 & 0.430 ** & & $1.619^{* *}$ & $0.475^{* *}$ & $1.499 * *$ & -0.956 & -0.129 & -0.267 & $1.263^{* *}$ & $0.604 * *$ & $4.284^{* *}$ & $4.237^{* *}$ & $1.292 * *$ \\
\hline IDN LI & -0.291 & $0.001^{* * *}$ & $0.001^{* * * *}$ & -13.194 & & $5.443^{* * *}$ & $0.001 * * *$ & $0.345^{* * *}$ & -0.053 & -0.179 & -0.071 & $2.421^{* * *}$ & $0.725^{* * *}$ & $3.544^{* * *}$ & $1.308^{* * *}$ & $1.029^{* * *}$ \\
\hline JPN RCA & 0.011 & 0.280 & 0.209 & 0.316 & 0.215 & & 0.001 & 0.164 & 0.013 & $1.663 *$ & 0.333 & 0.092 & 0.625 & $1.164 *$ & $1.589 *$ & 0.463 \\
\hline JPN RTA & -1.576 & $0.273 * *$ & -0.041 & -1.305 & -2.510 & & $0.001^{* *}$ & -0.083 & -2.555 & -10.881 & -2.141 & -0.594 & -4.553 & -1.958 & -0.773 & -1.682 \\
\hline JPN LI & -2.710 & $0.008^{* * *}$ & $0.004^{* * *}$ & -2.321 & -2.421 & & $0.001^{* * *}$ & $0.343^{* * *}$ & -0.068 & -0.692 & -0.195 & $0.239 * * *$ & $0.706^{* * *}$ & $4.978^{* * *}$ & $2.130^{* * *}$ & -0.927 \\
\hline LAO RCA & 0.001 & 0.001 & 0.001 & 0.002 & 0.001 & 0.001 & & 0.016 & 0.001 & 0.001 & 0.001 & 0.001 & 0.001 & 0.006 & 0.001 & 0.004 \\
\hline LAO RTA & -0.001 & -0.001 & -3.815 & -0.001 & -0.457 & -0.001 & & 0.015 * & -0.001 & -0.005 & -0.001 & -0.009 & -0.035 & -0.115 & -0.249 & -0.101 \\
\hline LAO LI & $0.023^{* * *}$ & $0.023 * * *$ & -0.133 & $0.801^{* * *}$ & -0.018 & $0.023^{* * *}$ & & $0.097^{* * *}$ & $0.023 * * *$ & $0.024^{* * *}$ & $0.023^{* * *}$ & $0.016^{* * *}$ & -0.018 & $0.094^{* * *}$ & -0.978 & -0.128 \\
\hline
\end{tabular}


Table A7. Cont.

\begin{tabular}{|c|c|c|c|c|c|c|c|c|c|c|c|c|c|c|c|c|}
\hline Countries & AUS & BRN & КHM & $\mathrm{CHN}$ & IDN & JPN & LAO & MYS & MMR & NZL & PHL & SGP & KOR & THA & VNM & RCEP \\
\hline MYS LI & $0.948^{* * *}$ & $0.617^{* * *}$ & -0.005 & -2.320 & -8.340 & $2.696^{* * *}$ & $0.001 * * *$ & & -1.641 & -0.387 & $0.327^{* * *}$ & $11.845^{* * *}$ & 0.063 *** & -3.072 & -0.729 & -0.610 \\
\hline MMR RCA & $70.785 *$ & 30.964 * & 0.001 & $6.487 *$ & 0.660 & $2.243 *$ & 0.001 & $22.844 *$ & & $1.167^{*}$ & 0.198 & 14.080 * & $2.623 *$ & $6.463 *$ & $1.470 *$ & $7.068 *$ \\
\hline MMR RTA & 70.740 ** & 30.957 ** & -0.001 & $6.357^{* *}$ & $0.547^{* *}$ & $2.221 * *$ & $0.001 * *$ & 22.833 ** & & $0.565^{* *}$ & $0.196^{* *}$ & 14.051 ** & $2.622 * *$ & $6.115^{* *}$ & 1.391 ** & $6.955^{* *}$ \\
\hline MMR LI & $0.087^{* * *}$ & $0.001^{* * *}$ & -0.001 & -0.341 & -0.137 & $0.038^{* * *}$ & $0.001 * * *$ & $0.354^{* * *}$ & & -0.030 & $0.001 * * *$ & $0.194^{* * *}$ & $0.039 * * *$ & -0.166 & -0.041 & $4.338^{* * *}$ \\
\hline NZL RCA & $5.074 *$ & $7.588 *$ & 0.001 & $4.383 *$ & 0.313 & $9.225 *$ & 0.004 & $1.516^{*}$ & 0.352 & & $1.269 *$ & $1.378^{*}$ & 1.917 * & $5.425^{*}$ & $3.340 *$ & $4.761 *$ \\
\hline NZL RTA & $4.919 * *$ & $7.585^{* *}$ & 0.001 ** & $4.101^{* *}$ & -0.029 & $8.289 * *$ & $0.004^{* *}$ & $1.439 * *$ & -0.751 & & $0.938^{* *}$ & 1.302 ** & -2.314 & $5.004^{* *}$ & $1.622 * *$ & $4.200 * *$ \\
\hline NZL LI & $4.894^{* * *}$ & $0.010^{* * *}$ & $0.001^{* * *}$ & $2.405^{* * *}$ & -0.237 & $1.120^{* * *}$ & $0.001^{* * *}$ & $0.065^{* * *}$ & -0.002 & & $0.078^{* * *}$ & -0.004 & -7.693 & -0.162 & -0.475 & $2.173^{* * *}$ \\
\hline PHL RCA & 0.243 & 0.365 & 0.017 & 0.542 & 0.692 & $1.219 *$ & 0.001 & 0.018 & 0.001 & 0.309 & & 0.112 & 0.117 & 0.816 & 0.785 & 0.667 \\
\hline PHL RTA & $0.176^{* *}$ & 0.350 ** & -0.026 & -1.157 & -0.064 & $0.645^{* *}$ & $0.001 * *$ & -0.070 & -0.169 & -1.136 & & -0.040 & -0.455 & 0.763 ** & -5.745 & -0.237 \\
\hline PHL LI & $0.211 * * *$ & $0.006^{* * *}$ & $0.001^{* * *}$ & -11.312 & -1.833 & $23.816^{* * *}$ & $0.001^{* * *}$ & -0.238 & -0.004 & -0.747 & & $0.156^{* * *}$ & -3.004 & $3.909 * * *$ & -10.959 & -0.692 \\
\hline SGP RCA & 0.045 & 1.060 * & 0.016 & 0.047 & 0.035 & 0.431 & 0.010 & 0.093 & 0.038 & 0.063 & 0.111 & & 0.121 & 0.147 & 0.258 & 0.117 \\
\hline SGP RTA & -0.321 & $1.041^{* *}$ & -0.279 & -0.138 & -1.249 & $0.262 * *$ & $0.009 * *$ & -0.248 & -11.097 & -1.596 & $0.029 * *$ & & $0.045^{* *}$ & -0.413 & -4.210 & -0.331 \\
\hline SGP LI & -0.007 & $1.862^{* * *}$ & -0.018 & -0.653 & -9.225 & $9.764^{* * *}$ & $0.001^{* * *}$ & -0.285 & -2.199 & -0.437 & $1.236^{* * *}$ & & $2.237^{* * *}$ & $0.173^{* * *}$ & -2.450 & -0.497 \\
\hline KOR RCA & 0.036 & 0.023 & 0.007 & 0.298 & 0.088 & 4.168 * & 0.028 & 0.078 & 0.001 & $7.482 *$ & 0.357 & 0.064 & & 4.388 * & 0.473 & 0.983 \\
\hline KOR RTA & -0.016 & $0.022 * *$ & -0.126 & -2.296 & -0.688 & $3.406^{* *}$ & $0.026^{* *}$ & -0.005 & -1.463 & $5.660 * *$ & -0.138 & -0.148 & & $3.308^{* *}$ & -3.369 & -0.469 \\
\hline KOR LI & -0.091 & $0.001 * * *$ & -0.002 & -25.921 & -1.341 & $22.389 * * *$ & $0.001^{* * *}$ & -0.004 & -0.075 & $1.566^{* * *}$ & $0.315^{* * *}$ & -0.079 & & $5.751^{* * *}$ & -2.509 & -0.505 \\
\hline THA RCA & 0.238 & 0.070 & 0.159 & 0.583 & 0.028 & $3.410 *$ & 0.083 & 0.864 & 0.136 & 0.066 & 0.052 & 0.472 & 0.950 & & 0.709 & $1.080 *$ \\
\hline THA RTA & -0.130 & 0.022 ** & -1.879 & -0.600 & -9.141 & $1.973^{* *}$ & $0.079 * *$ & $0.302^{* *}$ & -5.069 & -7.563 & -3.418 & $0.331 * *$ & -4.224 & & -7.631 & -1.054 \\
\hline THA LI & $0.410 * * *$ & -0.001 & $0.054^{* * *}$ & -1.682 & -10.427 & $20.763^{* * *}$ & $0.091^{* * *}$ & $2.387^{* * *}$ & -2.782 & -0.790 & -1.664 & $1.771^{* * *}$ & -5.782 & & -2.347 & -0.846 \\
\hline VNM RCA & $4.313 *$ & 17.490 * & 0.580 & $2.847 *$ & 0.392 & $4.454 *$ & 0.165 & $3.525 *$ & 0.097 & $1.644 *$ & $5.407^{*}$ & $4.310 *$ & $3.413 *$ & $7.437^{*}$ & & $3.594 *$ \\
\hline VNM RTA & $3.850 * *$ & $17.457^{* *}$ & $0.568^{* *}$ & $2.544^{* *}$ & -1.768 & 3.090 ** & $0.164^{* *}$ & $3.428^{* *}$ & -0.073 & -1.944 & $4.009 * *$ & 4.150 ** & $2.938^{* *}$ & $7.049 * *$ & & $3.050 * *$ \\
\hline
\end{tabular}

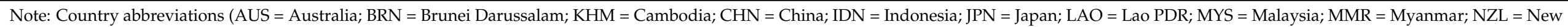

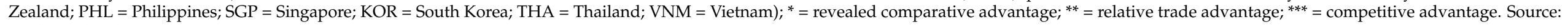

Authors' development. 
Table A8. RCA, RTA, and LI values for dried, salted, and smoked fish $\left(p_{3}\right)$ in RCEP countries, average in 2000-2019.

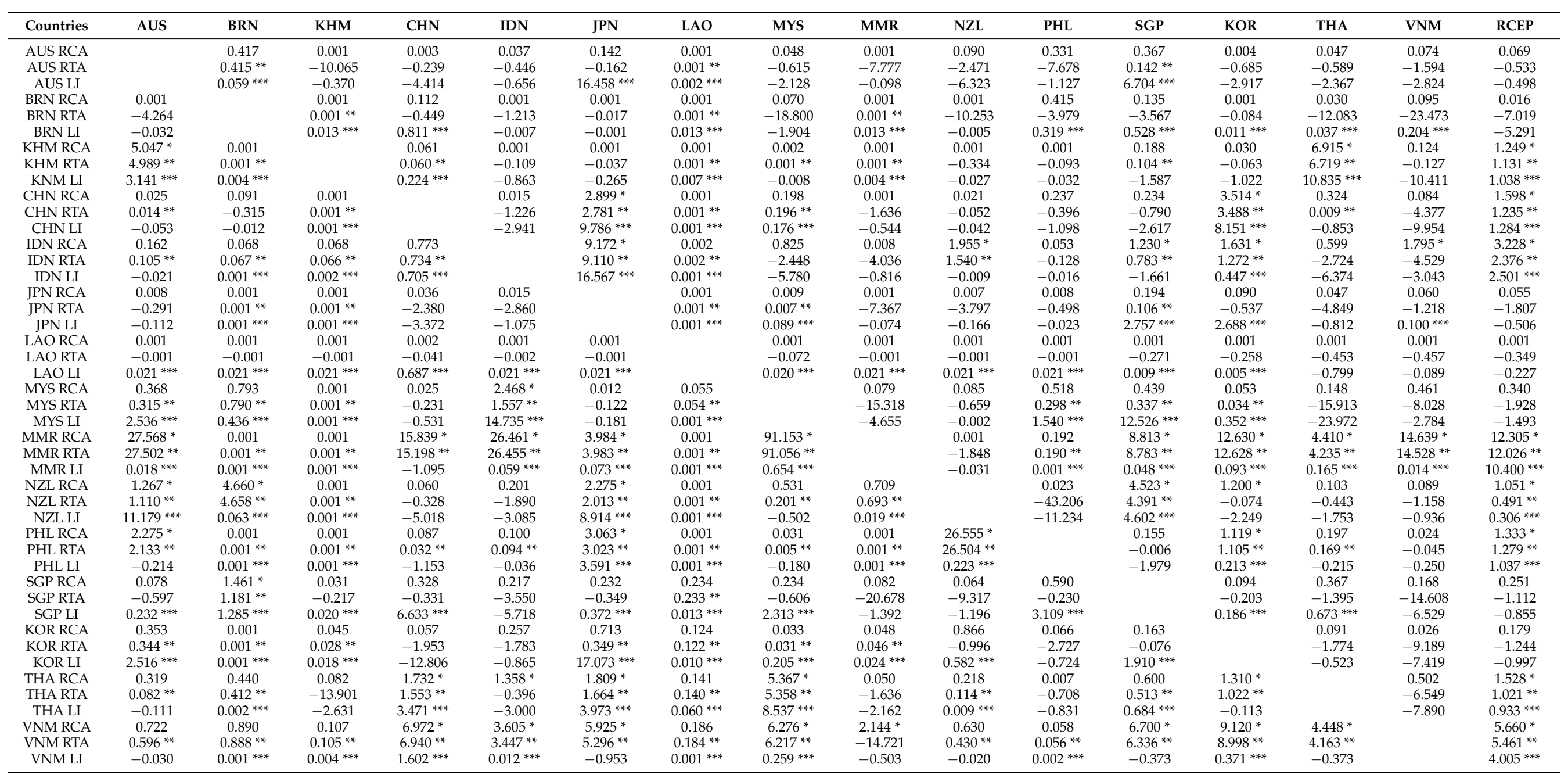

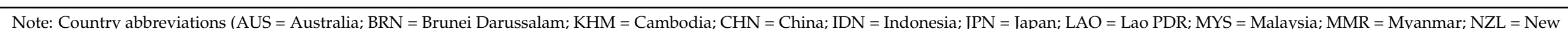

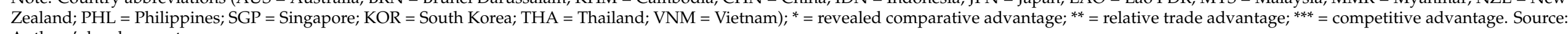
Authors' development. 
Table A9. RCA, RTA, and LI values for crustaceans, mollusks, and aquatic invertebrates $\left(p_{4}\right)$ in RCEP countries, average in $2000-2019$.

\begin{tabular}{|c|c|c|c|c|c|c|c|c|c|c|c|c|c|c|c|c|}
\hline Countries & AUS & BRN & КНM & $\mathrm{CHN}$ & IDN & JPN & LAO & MYS & MMR & NZL & PHL & SGP & KOR & THA & VNM & RCEP \\
\hline AUS RCA & & 0.157 & 0.099 & 0.306 & 0.090 & 0.843 & 0.138 & 0.446 & 0.056 & 0.121 & 0.017 & 0.945 & 0.010 & 0.368 & $18.382 *$ & 0.700 \\
\hline AUS RTA & & $0.024 * *$ & -0.038 & -0.703 & -1.067 & $0.639^{* *}$ & $0.136^{* *}$ & -0.660 & -71.383 & -1.518 & -0.093 & $0.905^{* *}$ & -0.043 & -2.225 & $12.915^{* *}$ & -0.356 \\
\hline AUS LI & & -0.043 & -0.002 & -8.403 & -2.209 & $14.479 * * *$ & $0.002 * * *$ & -2.901 & -0.738 & -4.143 & -0.019 & $2.655^{* * *}$ & -0.114 & -9.413 & $10.850^{* * *}$ & -0.299 \\
\hline BRN RCA & 0.136 & & 0.001 & 0.322 & 0.001 & 0.009 & 0.001 & 0.196 & 0.001 & 0.001 & 0.001 & 0.082 & 0.007 & 0.001 & 0.035 & 0.040 \\
\hline BRN RTA & -0.383 & & $0.001 * *$ & $0.084^{* *}$ & -0.473 & -0.010 & $0.001^{* *}$ & -3.317 & -20.362 & -2.510 & -0.099 & -0.979 & -0.013 & -0.341 & -3.410 & -1.351 \\
\hline BRN LI & $6.368^{* * *}$ & & $0.001^{* * *}$ & $5.600 * * *$ & -0.240 & $2.031^{* * *}$ & $0.001 * * *$ & -10.442 & -1.004 & -0.131 & -0.006 & -2.478 & $0.547^{* * *}$ & -0.260 & $0.015^{* * *}$ & -0.956 \\
\hline KHM RCA & 0.104 & 0.001 & & 0.982 & 0.001 & 0.145 & 0.001 & 0.002 & 0.001 & 0.001 & 0.001 & 0.034 & $2.445^{*}$ & $1.034 *$ & 0.168 & 0.499 \\
\hline KHM RTA & -0.015 & $0.001^{* *}$ & & $0.868 * *$ & -0.011 & 0.072 ** & 0.001 ** & -0.009 & $0.001 * *$ & -2.960 & -0.228 & 0.018 ** & $2.428 * *$ & $0.746^{* *}$ & -0.450 & $0.268^{* *}$ \\
\hline KNM LI & $0.177^{* * *}$ & $0.001 * * *$ & & $8.219 * * *$ & -0.074 & $2.631 * * *$ & $0.001 * * *$ & -0.048 & $0.001^{* * *}$ & -0.249 & -0.079 & $0.163^{* * *}$ & $10.453^{* * *}$ & -1.681 & -19.511 & $0.195^{* * *}$ \\
\hline CHN RTA & $0.525^{* *}$ & -0.289 & -1.316 & & -1.660 & 1.141 ** & $0.001^{* *}$ & 1.295 ** & -2.733 & -6.057 & $1.183^{* *}$ & $0.268^{* *}$ & $1.595^{* *}$ & $1.150^{* *}$ & -0.249 & $0.742 * *$ \\
\hline CHN LI & -0.922 & -0.017 & -0.101 & & -6.742 & $7.349^{* * *}$ & $0.001 * * *$ & $2.130^{* * *}$ & -1.508 & -6.698 & $1.409 * * *$ & $0.738^{* * *}$ & $6.627^{* * *}$ & -1.205 & -1.059 & $0.604^{* * *}$ \\
\hline IDN RCA & 0.842 & 0.277 & 0.008 & $1.742 *$ & & $5.449 *$ & 0.554 & 0.676 & 0.010 & 0.260 & 0.052 & 0.415 & 0.462 & 0.666 & 5.556 * & 2.234 * \\
\hline IDN RTA & $0.758^{* *}$ & $0.275^{* *}$ & $0.007^{* *}$ & $1.621 * *$ & & 5.416 ** & $0.552 * *$ & $0.586 * *$ & -2.284 & 0.183 ** & -0.246 & $0.393 * *$ & $0.427 * *$ & $0.641 * *$ & $5.339 * *$ & $2.167^{* * *}$ \\
\hline IDN LI & -0.209 & $0.001 * * *$ & $0.001 * * *$ & -1.558 & & $2.893^{* * *}$ & $0.001 * * *$ & -0.367 & -0.123 & -0.035 & -0.144 & -0.274 & -0.106 & -0.063 & -0.016 & $1.882^{* * *}$ \\
\hline JPN RCA & 0.156 & 0.009 & 0.080 & 0.272 & 0.020 & & 0.002 & 0.026 & 0.025 & 0.286 & 0.006 & 0.074 & 0.174 & 0.086 & 0.941 & 0.199 \\
\hline JPN RTA & -0.795 & -0.006 & -0.077 & -1.079 & -6.419 & & $0.002 * *$ & -0.945 & -37.887 & -2.116 & -2.678 & $0.071^{* *}$ & -1.837 & -6.582 & -15.885 & -2.495 \\
\hline JPN LI & -0.021 & -0.001 & $0.003 * * *$ & $4.594^{* * *}$ & -2.637 & & $0.001 * * *$ & -0.252 & -0.379 & $0.052 * * *$ & -0.403 & $0.426^{* * *}$ & $1.320^{* * *}$ & -1.660 & -1.043 & -2.007 \\
\hline LAO RCA & 0.001 & 0.001 & 0.001 & 0.001 & 0.001 & 0.001 & & 0.001 & 0.001 & 0.001 & 0.001 & 0.001 & 0.029 & 0.003 & 0.001 & 0.002 \\
\hline LAO RTA & -0.212 & -0.001 & -0.001 & -0.002 & -1.515 & -0.002 & & -0.069 & -0.001 & -41.659 & -0.001 & -0.009 & $0.010 * *$ & -0.038 & -0.039 & -0.043 \\
\hline LAO LI & -0.165 & $0.001 * * *$ & $0.001^{* * *}$ & -0.057 & -0.351 & -0.006 & & -0.017 & $0.001^{* * *}$ & -1.470 & $0.001^{* * *}$ & -0.019 & $0.985^{* * *}$ & $1.064^{* * *}$ & $0.034^{* * *}$ & -0.087 \\
\hline MYS RTA & 0.430 ** & $0.382 * *$ & $0.007^{* *}$ & -1.054 & -1.237 & 0.763 ** & $0.040 * *$ & & -29.987 & -0.906 & -0.087 & $0.372 * *$ & $1.035^{* *}$ & -0.301 & $0.998^{* *}$ & -0.098 \\
\hline MYS LI & $3.344^{* * *}$ & $0.201^{* * *}$ & $0.001^{* * *}$ & -19.627 & -6.727 & $11.289 * * *$ & $0.001 * * *$ & & -3.955 & -0.355 & -0.128 & $8.552 * * *$ & $5.401 * * *$ & -1.690 & $3.694^{* * * *}$ & -0.105 \\
\hline MMR RCA & $63.104 *$ & $20.466^{*}$ & 0.001 & $3.998 *$ & $4.051 *$ & $31.988 *$ & 0.001 & $12.877^{*}$ & & $10.742 *$ & 0.137 & $9.130 *$ & $1.615^{*}$ & $2.787 *$ & $14.463 *$ & $6.651 *$ \\
\hline MMR RTA & $63.052 * *$ & 20.403 ** & 0.001 ** & $3.970 * *$ & $4.049 * *$ & $31.971 * *$ & $0.001^{* *}$ & $12.861 * *$ & & $9.780 * *$ & $0.135^{* *}$ & $9.125 * *$ & $1.611^{* *}$ & $2.524^{* *}$ & $14.462 * *$ & $6.598^{* *}$ \\
\hline MMR LI & $0.032 * * *$ & $0.001 * * *$ & $0.001^{* * *}$ & $0.019 * * *$ & $0.008^{* * *}$ & $0.592 * * *$ & $0.001 * * *$ & $0.081^{* * *}$ & & -0.048 & $0.001^{* * *}$ & $0.093^{* * *}$ & $0.006^{* * *}$ & -0.822 & $0.038^{* * *}$ & $5.883^{* * *}$ \\
\hline NZL RCA & $1.266^{*}$ & $2.472 *$ & $1.831^{*}$ & $5.453 *$ & 0.061 & $2.186^{*}$ & $8.633 *$ & $1.063 *$ & 0.394 & & 0.275 & $1.589 *$ & $2.521 *$ & $3.262 *$ & 0.744 & $2.602 *$ \\
\hline NZL RTA & $1.102 * *$ & $2.468 * *$ & $1.829 * *$ & 4.498 ** & -0.240 & $1.956^{* *}$ & $8.629 * *$ & $0.677^{* *}$ & -6.554 & & $0.266^{* *}$ & 1.580 ** & $0.181^{* *}$ & $0.784^{* *}$ & -9.697 & $1.859^{* *}$ \\
\hline NZL LI & $2.992 * * *$ & $0.012^{* * *}$ & $0.004^{* * *}$ & $8.824^{* * *}$ & -0.306 & $2.647^{* * *}$ & $0.006^{* * *}$ & -0.451 & -0.020 & & $0.085^{* * *}$ & $0.655^{* * *}$ & -4.141 & -4.713 & -5.595 & $1.436^{* * *}$ \\
\hline PHL RCA & 0.050 & 0.087 & 0.111 & 0.427 & 0.239 & $1.719 *$ & 0.001 & 0.125 & 0.004 & 0.003 & & 0.441 & 2.244 * & 0.154 & $1.389 *$ & 1.011 * \\
\hline PHL RTA & $0.039 * *$ & $0.085^{* *}$ & $0.110^{* *}$ & -0.194 & 0.176 ** & $1.709 * *$ & $0.001 * *$ & 0.108 ** & -0.369 & -0.331 & & $0.424^{* *}$ & $2.178^{* *}$ & $0.134^{* *}$ & -0.095 & $0.798^{* *}$ \\
\hline PHL LI & -0.014 & $0.001^{* * *}$ & $0.002^{* * *}$ & -17.394 & -0.524 & $18.725^{* * *}$ & $0.001^{* * *}$ & $0.085^{* * *}$ & -0.028 & -0.524 & & $1.689^{* * *}$ & $4.902 * * *$ & $0.072 * * *$ & -6.991 & $0.504^{* * *}$ \\
\hline SGP RCA & 0.032 & 0.506 & 0.014 & 0.016 & 0.017 & 0.030 & 0.006 & 0.100 & 0.013 & 0.007 & 0.028 & & 0.011 & 0.020 & 0.150 & 0.045 \\
\hline SGP RTA & -0.966 & $0.404^{* *}$ & -0.018 & -0.301 & -0.576 & -0.136 & $0.005^{* *}$ & -0.368 & -15.430 & -2.030 & -0.431 & & -0.054 & -0.552 & -3.098 & -0.420 \\
\hline SGP LI & -0.488 & $1.280^{* * *}$ & $0.044^{* * *}$ & -2.015 & -1.486 & -0.036 & $0.001 * * *$ & $5.072^{* * *}$ & -1.686 & -0.395 & -0.213 & & $0.048^{* * *}$ & -1.092 & $0.967^{* * *}$ & -0.328 \\
\hline KOR RCA & 0.046 & 0.003 & 0.014 & 0.187 & 0.035 & $1.606^{*}$ & 0.016 & 0.070 & 0.001 & $3.765^{*}$ & 0.086 & 0.059 & & 0.163 & 0.104 & 0.372 \\
\hline KOR RTA & $0.035^{* *}$ & -0.009 & -3.028 & -1.751 & -0.451 & $1.380 * *$ & $0.015^{* *}$ & -0.975 & -2.572 & $3.214^{* *}$ & -1.155 & $0.035^{* *}$ & & -6.714 & -13.726 & -1.183 \\
\hline KOR LI & $0.149 * * *$ & -0.002 & -0.041 & -7.959 & -0.529 & $21.548^{* * *}$ & $0.001 * * *$ & -0.959 & -0.093 & $1.883^{* * *}$ & -0.219 & $0.384^{* * *}$ & & -3.339 & -10.821 & -0.846 \\
\hline THA RCA & $2.027^{*}$ & 0.198 & 0.232 & 0.892 & 0.019 & $6.235 *$ & 0.034 & 0.266 & 0.093 & $1.451 *$ & 0.008 & 0.650 & $5.930 *$ & & $2.128 *$ & 2.120 * \\
\hline THA RTA & $1.715^{* *}$ & $0.196^{* *}$ & -1.133 & 0.130 ** & -0.898 & $6.097^{* *}$ & $0.029 * *$ & -0.147 & -2.040 & -0.916 & -0.196 & $0.640 * *$ & $5.727 * *$ & & -2.709 & $1.565^{* *}$ \\
\hline
\end{tabular}


Table A9. Cont.

\begin{tabular}{|c|c|c|c|c|c|c|c|c|c|c|c|c|c|c|c|c|}
\hline Countries & AUS & BRN & KHM & CHN & IDN & JPN & LAO & MYS & MMR & NZL & PHL & SGP & KOR & THA & VNM & RCEP \\
\hline THA LI & $1.381^{* * *}$ & $0.003^{* * *}$ & -0.174 & -8.240 & -2.585 & $16.011 * * *$ & $0.010^{* * *}$ & -1.823 & -2.944 & -0.432 & -0.252 & $0.855^{* * *}$ & $2.463^{* * *}$ & & -4.273 & $0.983^{* * *}$ \\
\hline VNM RCA & 4.033 * & 0.433 & 0.441 & $4.687^{*}$ & 0.176 & $15.592 *$ & 0.044 & $1.576^{*}$ & 0.008 & $8.564^{*}$ & $1.062 *$ & $3.065 *$ & 12.019 * & $4.625 *$ & & $7.258 *$ \\
\hline VNM RTA & $3.492 * *$ & 0.401 ** & $0.248^{* *}$ & 4.538 ** & -3.110 & $15.375^{* *}$ & $0.042 * *$ & $1.004^{* *}$ & -12.277 & $8.206^{* *}$ & $0.221 * *$ & $2.900 * *$ & 11.977 ** & $4.112 * *$ & & $6.959 * *$ \\
\hline VNM LI & -0.034 & -0.001 & -0.013 & $0.025^{* * *}$ & -2.998 & $3.731^{* * *}$ & $0.001 * * *$ & -0.709 & -0.450 & $0.002 * * *$ & -0.188 & -0.172 & $1.763^{* * *}$ & -0.957 & & $4.296^{* * *}$ \\
\hline
\end{tabular}

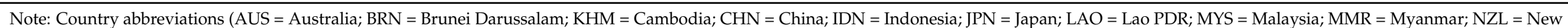

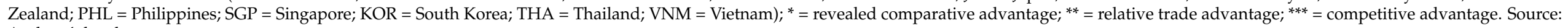

Authors' development.

Table A10. RCA, RTA, and LI values for prepared and preserved fish and aquatic invertebrates $\left(p_{5}\right)$ in RCEP countries, average in 2000-2019.

\begin{tabular}{|c|c|c|c|c|c|c|c|c|c|c|c|c|c|c|c|c|}
\hline Countries & AUS & BRN & КНM & $\mathrm{CHN}$ & IDN & JPN & LAO & MYS & MMR & NZL & PHL & SGP & KOR & THA & VNM & RCEP \\
\hline AUS RCA & & 0.011 & 0.148 & 0.404 & 0.007 & 0.091 & 0.007 & 0.120 & 0.006 & 0.214 & 0.003 & 0.903 & 0.001 & 0.043 & $4.245^{*}$ & 0.305 \\
\hline AUS RTA & & $0.009 * *$ & -0.163 & -0.158 & -1.232 & -0.019 & 0.006 ** & -1.568 & -36.428 & -1.717 & -1.720 & $0.774 * *$ & -0.229 & -11.397 & -1.014 & -1.392 \\
\hline AUS LI & & $0.001^{* * *}$ & $0.001 * * *$ & $13.833^{* * *}$ & -1.063 & $2.332^{* * *}$ & $0.001 * * *$ & -2.071 & -0.158 & -0.927 & -0.207 & $3.961 * * *$ & -0.348 & -18.825 & $3.473^{* * *}$ & -0.994 \\
\hline BRN RCA & 0.001 & & 0.001 & 0.005 & 0.001 & 0.001 & 0.001 & 0.049 & 0.001 & 0.001 & 0.009 & 0.008 & 0.001 & 0.004 & 0.001 & 0.002 \\
\hline BRN LI & $0.057^{* * *}$ & & $0.001 * * *$ & $0.071^{* * *}$ & -0.018 & -0.010 & $0.001^{* * *}$ & $0.076^{* * *}$ & $0.001^{* * *}$ & -0.016 & -0.028 & -0.017 & -0.014 & -0.090 & -0.011 & -1.053 \\
\hline KHM RCA & 0.281 & 0.001 & & 0.144 & 0.001 & 0.001 & 0.021 & 0.034 & 0.001 & 0.001 & 0.001 & 0.001 & 0.005 & 0.284 & 0.336 & 0.133 \\
\hline KHM RTA & $0.146^{* *}$ & $0.001 * *$ & & -0.159 & -0.014 & -1.603 & 0.020 ** & -0.031 & $0.001 * *$ & -22.524 & -0.076 & -0.037 & -0.111 & -1.432 & -1.261 & -0.791 \\
\hline KNM LI & $0.397^{* * *}$ & $0.001^{* * *}$ & & $0.560^{* * *}$ & -0.003 & -0.347 & $0.001 * * *$ & $0.054^{* * *}$ & $0.001^{* * *}$ & -0.071 & -0.001 & -0.021 & -0.027 & -1.384 & $0.842^{* * *}$ & -0.504 \\
\hline CHN RCA & $1.095 *$ & 0.183 & 0.318 & & 0.345 & $4.661 *$ & 0.583 & $1.728 *$ & 0.023 & $1.346^{*}$ & 0.600 & 0.827 & $1.145^{*}$ & $1.487^{*}$ & 0.275 & $2.076^{*}$ \\
\hline CHN LI & -2.918 & $0.001^{* * *}$ & -0.001 & & -0.830 & $7.610^{* * *}$ & $0.007^{* * *}$ & $0.341^{* * *}$ & -0.243 & -2.495 & -0.275 & $0.398^{* * *}$ & $0.456^{* * *}$ & -1.605 & -0.447 & $1.553^{* * *}$ \\
\hline IDN RCA & $1.267^{*}$ & 0.218 & 0.006 & 0.185 & & $1.458 *$ & 0.001 & 0.294 & 0.016 & 0.165 & 0.041 & 0.123 & 0.118 & 1.241 * & 0.317 & 0.631 \\
\hline IDN RTA & $1.261 * *$ & $0.216^{* *}$ & 0.005 ** & -0.037 & & $1.431^{* *}$ & $0.001 * *$ & -0.012 & 0.015 ** & $0.163^{* *}$ & -0.127 & $0.010 * *$ & $0.100 * *$ & $1.102^{* *}$ & $0.251 * *$ & 0.501 ** \\
\hline IDN LI & $2.213^{* * *}$ & $0.008^{* * *}$ & $0.001^{* * *}$ & -10.143 & & $15.923 * * *$ & $0.001^{* * *}$ & -3.642 & $0.003^{* * *}$ & $0.029^{* * *}$ & -0.162 & -5.152 & $0.237^{* * *}$ & $0.620^{* * *}$ & $0.065^{* * *}$ & $0.472^{* * *}$ \\
\hline JPN RCA & 0.089 & 0.010 & $1.510 *$ & 0.062 & 0.020 & & $1.589 *$ & 0.078 & 0.005 & 0.143 & 0.023 & 0.205 & 0.058 & 0.060 & 0.199 & 0.078 \\
\hline JPN RTA & -0.065 & $0.009 * *$ & $1.509 * *$ & -4.190 & -2.941 & & $1.586^{* *}$ & 0.019 ** & -1.756 & -0.884 & -1.976 & $0.201 * *$ & -1.479 & -11.857 & -9.289 & -3.461 \\
\hline JPN LI & $0.252 * * *$ & $0.001^{* * * *}$ & $0.067^{* * *}$ & -1.072 & -0.248 & & $0.025 * * *$ & $0.240^{* * *}$ & -0.004 & $0.055^{* * * *}$ & -0.023 & $0.961^{* * *}$ & $0.449^{* * *}$ & -0.636 & -0.066 & -2.495 \\
\hline LAO RCA & 0.001 & 0.001 & 0.001 & 0.001 & 0.001 & 0.001 & & 0.001 & 0.001 & 0.001 & 0.001 & 0.001 & 0.218 & 0.001 & 0.004 & 0.003 \\
\hline LAO RTA & -0.007 & -0.001 & -0.031 & -0.562 & -0.001 & -2.655 & & -0.001 & -0.001 & -0.001 & -0.001 & -0.026 & $0.107^{* *}$ & -0.557 & -0.812 & -0.596 \\
\hline LAO LI & -0.001 & -0.001 & -0.001 & -0.137 & -0.001 & -0.070 & & -0.001 & -0.001 & -0.001 & -0.001 & -0.001 & $0.582 * * *$ & -0.442 & $0.067^{* * *}$ & -0.604 \\
\hline MYS LI & $15.993 * * *$ & $1.590^{* * *}$ & $0.018^{* * *}$ & -16.830 & -0.725 & $1.745^{* * *}$ & $0.001 * * *$ & & -0.997 & $0.040^{* * *}$ & -0.444 & $15.568^{* * *}$ & $0.763^{* * *}$ & -14.773 & -1.948 & -0.106 \\
\hline MMR RCA & $4.423 *$ & 0.001 & 0.001 & $1.158^{*}$ & 0.001 & 0.258 & 0.001 & 0.394 & & $5.702 *$ & 0.352 & 0.231 & 0.302 & 0.515 & 0.547 & 0.695 \\
\hline MMR RTA & $4.397 * *$ & $0.001 * *$ & $0.001 * *$ & 1.149 ** & -0.053 & $0.217^{* *}$ & $0.001 * *$ & $0.375^{* *}$ & & $5.692 * *$ & $0.345^{* *}$ & $0.214^{* *}$ & $0.295 * *$ & -0.186 & 0.526 ** & $0.583^{* *}$ \\
\hline MMR LI & $0.423^{* * *}$ & $0.001^{* * *}$ & $0.001 * * *$ & $13.706^{* * *}$ & -0.501 & $0.068^{* * *}$ & $0.001 * * *$ & $0.262 * * *$ & & $0.042 * * *$ & $0.039^{* * *}$ & -0.408 & $0.292^{* * *}$ & -14.048 & $0.126^{* * *}$ & $0.735^{* * *}$ \\
\hline NZL RCA & $1.537 *$ & $3.892 *$ & $18.029 *$ & $2.482 *$ & 0.002 & 0.572 & 0.001 & 0.696 & 0.004 & & 0.043 & $6.343 *$ & 1.501 * & $1.413^{*}$ & 0.081 & $1.673 *$ \\
\hline NZL RTA & $1.298^{* *}$ & $3.881^{* *}$ & $17.884^{* *}$ & $1.639^{* *}$ & -0.189 & $0.410^{* *}$ & $0.001^{* *}$ & $0.188^{* *}$ & -6.019 & & -1.022 & $6.283^{* *}$ & $1.184^{* *}$ & -10.492 & -5.367 & 0.601 ** \\
\hline
\end{tabular}


Table A10. Cont.

\begin{tabular}{|c|c|c|c|c|c|c|c|c|c|c|c|c|c|c|c|c|}
\hline Countries & AUS & BRN & КНM & $\mathrm{CHN}$ & IDN & JPN & LAO & MYS & MMR & NZL & PHL & SGP & KOR & THA & VNM & RCEP \\
\hline NZL LI & $11.266^{* * *}$ & $0.044^{* * *}$ & $0.099 * * *$ & $10.380^{* * *}$ & -0.210 & $1.407^{* * *}$ & $0.001^{* * *}$ & -0.525 & -0.021 & & -0.163 & $5.970 * * *$ & $1.849^{* * *}$ & -27.116 & -2.981 & $0.583^{* * *}$ \\
\hline PHL RCA & $1.398 *$ & $6.646^{*}$ & 0.064 & 0.096 & 0.078 & 0.745 & 0.001 & 0.258 & 0.156 & $1.013 *$ & & 0.483 & 0.214 & 0.366 & 0.253 & 0.452 \\
\hline PHL RTA & $1.374^{* *}$ & $6.637 * *$ & 0.061 ** & -0.016 & $0.026^{* *}$ & $0.740^{* *}$ & 0.001 ** & $0.190^{* *}$ & -0.016 & 1.012 ** & & 0.450 ** & $0.209 * *$ & $0.194^{* *}$ & 0.221 ** & $0.395^{* *}$ \\
\hline PHL LI & $1.018^{* * *}$ & $0.088^{* * *}$ & $0.002 * * *$ & -8.970 & -1.481 & $13.159 * * *$ & $0.001^{* * *}$ & -1.058 & -0.030 & $0.103 * * *$ & & $1.960 * * *$ & $0.711^{* * *}$ & -5.302 & -0.199 & $0.499^{* * *}$ \\
\hline SGP RCA & 0.087 & 0.677 & 0.028 & 0.006 & 0.095 & 0.007 & 0.055 & 0.128 & 0.022 & 0.049 & 0.047 & & 0.032 & 0.026 & 0.189 & 0.070 \\
\hline SGP RTA & -1.248 & $0.668^{* *}$ & $0.026^{* *}$ & -0.690 & -0.162 & -0.190 & 0.053 ** & -0.278 & -0.551 & -7.720 & -0.302 & & -0.018 & -1.492 & -2.323 & -0.490 \\
\hline SGP LI & $0.226^{* * *}$ & $1.294^{* * *}$ & $0.072 * * *$ & -7.230 & $4.343^{* * *}$ & -1.242 & $0.007^{* * *}$ & $5.537 * * *$ & $0.033^{* * *}$ & -1.395 & $0.138^{* * *}$ & & $0.613^{* * *}$ & -3.839 & $1.443^{* * *}$ & -0.394 \\
\hline KOR RCA & 0.156 & 0.158 & 0.120 & 0.097 & 0.017 & $1.280 *$ & 0.085 & 0.060 & 0.056 & 0.238 & 0.050 & 0.044 & & 0.065 & 0.045 & 0.254 \\
\hline KOR RTA & $0.155^{* *}$ & $0.152 * *$ & 0.112 ** & -0.621 & -0.340 & $1.215^{* *}$ & -0.174 & -0.050 & -0.511 & -3.045 & -1.092 & -0.005 & & -3.706 & -5.920 & -0.403 \\
\hline KOR LI & $1.019^{* * *}$ & $0.016^{* * *}$ & $0.043^{* * *}$ & -9.718 & -1.273 & $31.011^{* * *}$ & $0.004^{* * *}$ & -0.006 & -0.033 & -1.196 & -1.032 & $0.395^{* * *}$ & & -5.598 & -13.632 & -0.357 \\
\hline THA RCA & $11.172 *$ & $4.023 *$ & $1.582 *$ & 0.590 & 0.125 & $10.248 *$ & 0.491 & $1.075 *$ & $1.460 *$ & $11.174 *$ & 0.306 & $1.304 *$ & $3.639 *$ & & 0.318 & $3.717 *$ \\
\hline THA RTA & $11.149^{* *}$ & $4.001 * *$ & $1.278^{* *}$ & -0.097 & -1.296 & $10.192 * *$ & $0.488^{* *}$ & 0.913 ** & $0.942 * *$ & $7.881 * *$ & -0.104 & $1.294 * *$ & $3.587 * *$ & & -3.587 & $3.287^{* *}$ \\
\hline THA LI & $3.694^{* * *}$ & $0.021^{* * *}$ & $0.192^{* * *}$ & -6.599 & -2.923 & $9.090^{* * *}$ & $0.058^{* * *}$ & -0.155 & -0.358 & -0.152 & -0.315 & $0.552 * * *$ & $0.500^{* * *}$ & & -3.603 & $2.840^{* * *}$ \\
\hline VNM RCA & $5.483 *$ & 0.090 & $1.493 *$ & 0.250 & 0.066 & 8.018 * & 0.671 & 0.468 & 0.034 & $6.165 *$ & 0.042 & $1.410 *$ & $5.101 *$ & $3.357 *$ & & $3.254 *$ \\
\hline VNM RTA & $5.469 * *$ & $0.084^{* *}$ & $1.217^{* *}$ & $0.201 * *$ & 0.028 ** & 7.979 ** & $0.665^{* *}$ & $0.420 * *$ & -0.802 & $6.057^{* *}$ & -0.127 & $1.329 * *$ & 5.090 ** & 3.200 ** & & $3.203 * *$ \\
\hline VNM LI & $0.439^{* * *}$ & $0.001^{* * *}$ & -0.053 & -1.392 & -0.083 & $1.875^{* * *}$ & $0.006^{* * *}$ & -0.139 & -0.076 & $0.031^{* * *}$ & -0.118 & -0.375 & $0.638^{* * *}$ & -0.752 & & $2.981^{* * *}$ \\
\hline
\end{tabular}

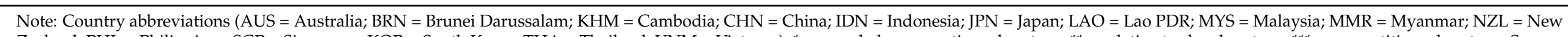

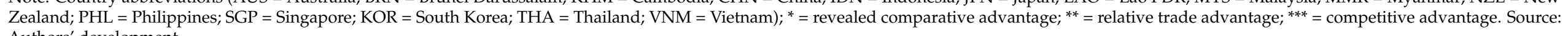
Authors' development. 


\section{Appendix C}

Table A11. Degrees of trade potentials and advantages of RCEP countries in trade in fresh, chilled, and frozen fish $\left(p_{2}\right)$ in $2000-2019$.

\begin{tabular}{|c|c|c|c|c|c|c|c|c|c|c|c|c|c|c|c|c|}
\hline Countries & AUS & BRN & КНM & $\mathrm{CHN}$ & IDN & JPN & LAO & MYS & MMR & NZL & PHL & SGP & KOR & THA & VNM & RCEP \\
\hline AUS & & A & $\mathrm{D}$ & W & D & $\mathrm{S}$ & A & D & D & D & W & A & A & D & D & D \\
\hline BRN & D & & D & W & W & W & A & D & D & D & D & D & D & W & D & D \\
\hline KHM & $\mathrm{A}$ & A & & A & W & $\mathrm{A}$ & S & A & $\mathrm{D}$ & $\mathrm{A}$ & A & A & A & S & D & A \\
\hline $\mathrm{CHN}$ & W & $\mathrm{D}$ & $\mathrm{D}$ & & W & S & W & A & D & D & S & A & S & A & D & S \\
\hline IDN & W & $\mathrm{A}$ & $\mathrm{W}$ & A & & S & A & S & D & D & D & $S$ & A & $\mathrm{S}$ & S & S \\
\hline JPN & D & A & W & D & $\mathrm{D}$ & & A & W & D & W & D & $\mathrm{W}$ & W & W & W & D \\
\hline $\mathrm{LAO}$ & W & W & D & W & D & W & & A & W & W & W & W & D & W & D & D \\
\hline MYS & $\mathrm{W}$ & A & $\mathrm{D}$ & D & $\mathrm{D}$ & W & W & & D & $\mathrm{D}$ & A & A & $\mathrm{W}$ & D & D & D \\
\hline MMR & $S$ & $S$ & $\mathrm{D}$ & W & W & S & A & S & & $\mathrm{W}$ & A & S & S & W & $\mathrm{W}$ & $S$ \\
\hline NZL & S & S & A & S & $\mathrm{D}$ & S & $\mathrm{S}$ & S & $\mathrm{D}$ & & S & W & W & W & W & S \\
\hline PHL & A & S & W & $\mathrm{D}$ & D & S & A & D & D & D & & W & D & A & $\mathrm{D}$ & $\mathrm{D}$ \\
\hline SGP & D & $S$ & D & D & $\mathrm{D}$ & A & A & D & D & D & A & & A & W & W & W \\
\hline KOR & $\mathrm{D}$ & A & $\mathrm{D}$ & D & D & S & A & D & D & S & W & D & & S & D & D \\
\hline THA & W & W & W & D & D & S & A & A & D & D & $\mathrm{D}$ & A & D & & D & W \\
\hline VNM & $\mathrm{S}$ & $\mathrm{S}$ & A & $\mathrm{S}$ & D & W & A & S & D & W & S & $\mathrm{S}$ & W & S & & $\mathrm{S}$ \\
\hline
\end{tabular}

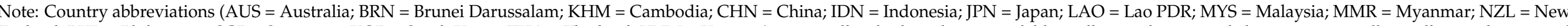

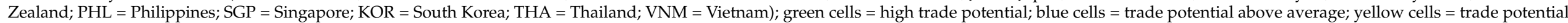
below average; red cells = low trade potential; $\mathrm{S}$ = strong advantage; $\mathrm{A}$ = average advantage; $\mathrm{W}$ = weak advantage; $\mathrm{D}=$ disadvantage. Source: Authors' development. 
Table A12. Degrees of trade potentials and advantages of RCEP countries in trade in dried, salted, and smoked fish $\left(p_{3}\right)$ in $2000-2019$.

\begin{tabular}{|c|c|c|c|c|c|c|c|c|c|c|c|c|c|c|c|c|}
\hline Countries & AUS & BRN & KHM & $\mathrm{CHN}$ & IDN & JPN & LAO & MYS & MMR & NZL & PHL & SGP & KOR & THA & VNM & RCEP \\
\hline AUS & & $\mathrm{A}$ & $\mathrm{D}$ & $\mathrm{D}$ & $\mathrm{D}$ & W & $\mathrm{A}$ & $\mathrm{D}$ & $\mathrm{D}$ & $\mathrm{D}$ & $\mathrm{D}$ & $\mathrm{A}$ & $\mathrm{D}$ & $\mathrm{D}$ & $\mathrm{D}$ & $\mathrm{D}$ \\
\hline BRN & $\mathrm{D}$ & & A & W & $\mathrm{D}$ & D & $\mathrm{A}$ & D & $\mathrm{A}$ & D & W & W & W & W & W & D \\
\hline KHM & S & A & & A & $\mathrm{D}$ & $\mathrm{D}$ & A & W & $\mathrm{A}$ & $\mathrm{D}$ & $\mathrm{D}$ & W & $\mathrm{D}$ & S & D & S \\
\hline $\mathrm{CHN}$ & $\mathrm{W}$ & $\mathrm{D}$ & A & & $\mathrm{D}$ & S & A & $\mathrm{A}$ & D & D & D & $\mathrm{D}$ & S & W & D & S \\
\hline IDN & W & A & A & A & & S & A & D & D & W & D & W & S & D & W & S \\
\hline JPN & $\mathrm{D}$ & A & A & D & D & & A & A & D & $\mathrm{D}$ & D & A & W & D & W & D \\
\hline LAO & W & W & W & W & W & W & & W & W & W & W & W & W & D & D & $\mathrm{D}$ \\
\hline MYS & A & A & A & D & S & D & A & & D & D & A & A & A & D & D & D \\
\hline MMR & S & $\mathrm{A}$ & A & W & $S$ & $S$ & A & S & & D & A & $S$ & $\mathrm{~S}$ & S & S & $S$ \\
\hline NZL & S & S & A & $\mathrm{D}$ & $\mathrm{D}$ & $\mathrm{S}$ & A & W & A & & D & S & W & D & D & S \\
\hline PHL & W & A & A & W & W & S & A & W & $\mathrm{A}$ & S & & $\mathrm{D}$ & $\mathrm{S}$ & W & $\mathrm{D}$ & $S$ \\
\hline SGP & W & $S$ & W & W & D & W & A & W & D & D & W & & W & W & D & D \\
\hline KOR & A & A & $\mathrm{A}$ & D & D & A & A & A & A & $\mathrm{W}$ & D & W & & D & D & D \\
\hline THA & W & A & D & S & W & S & A & S & D & A & D & A & W & & $\mathrm{D}$ & S \\
\hline VNM & W & A & A & S & S & W & A & S & W & W & A & W & S & W & & S \\
\hline
\end{tabular}

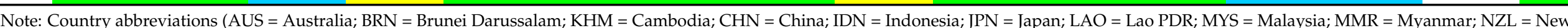

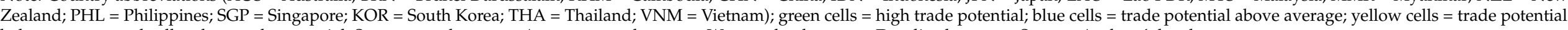
below average; red cells = low trade potential; $\mathrm{S}=$ strong advantage; $\mathrm{A}=$ average advantage; $\mathrm{W}=$ weak advantage; $\mathrm{D}=$ disadvantage. Source: Authors' development. 
Table A13. Degrees of trade potentials and advantages of RCEP countries in trade in crustaceans, mollusks, and aquatic invertebrates $\left(p_{4}\right)$ in $2000-2019$.

\begin{tabular}{|c|c|c|c|c|c|c|c|c|c|c|c|c|c|c|c|c|}
\hline Countries & AUS & BRN & КНМ & $\mathrm{CHN}$ & IDN & JPN & LAO & MYS & MMR & NZL & PHL & SGP & KOR & THA & VNM & RCEP \\
\hline AUS & & $\mathrm{W}$ & $\mathrm{D}$ & $\mathrm{D}$ & $\mathrm{D}$ & $\mathrm{A}$ & $\mathrm{A}$ & $\mathrm{D}$ & $\mathrm{D}$ & $\mathrm{D}$ & $\mathrm{D}$ & $\mathrm{A}$ & $\mathrm{D}$ & $\mathrm{D}$ & $\mathrm{S}$ & $\mathrm{D}$ \\
\hline BRN & W & & $\mathrm{A}$ & A & $\mathrm{D}$ & $\mathrm{W}$ & $\mathrm{A}$ & $\mathrm{D}$ & $\mathrm{D}$ & D & $\mathrm{D}$ & $\mathrm{D}$ & $\mathrm{W}$ & $\mathrm{D}$ & W & $\mathrm{D}$ \\
\hline KHM & $\mathrm{W}$ & A & & A & D & A & A & D & A & $\mathrm{D}$ & $\mathrm{D}$ & A & $\mathrm{S}$ & W & D & A \\
\hline $\mathrm{CHN}$ & $\mathrm{W}$ & $\mathrm{D}$ & D & & D & $\mathrm{S}$ & A & $\mathrm{S}$ & D & $\mathrm{D}$ & $\mathrm{S}$ & A & S & W & $\mathrm{D}$ & S \\
\hline IDN & W & A & A & W & & S & A & W & D & W & D & W & W & W & W & S \\
\hline JPN & D & $\mathrm{D}$ & $\mathrm{W}$ & W & D & & A & $\mathrm{D}$ & $\mathrm{D}$ & W & $\mathrm{D}$ & A & W & $\mathrm{D}$ & D & $\mathrm{D}$ \\
\hline $\mathrm{LAO}$ & $\mathrm{D}$ & $\mathrm{W}$ & W & D & D & $\mathrm{D}$ & & $\mathrm{D}$ & W & $\mathrm{D}$ & W & D & $\mathrm{A}$ & W & W & $\mathrm{D}$ \\
\hline MYS & $\mathrm{A}$ & $\mathrm{A}$ & A & $\mathrm{D}$ & $\mathrm{D}$ & A & A & & D & $\mathrm{D}$ & D & A & S & $\mathrm{D}$ & $\mathrm{S}$ & D \\
\hline MMR & $\mathrm{S}$ & $S$ & A & S & S & S & A & S & & $\mathrm{W}$ & A & S & $S$ & W & S & $S$ \\
\hline NZL & $S$ & S & $S$ & S & D & S & $S$ & $\mathrm{~W}$ & D & & A & $\mathrm{S}$ & W & W & $\mathrm{D}$ & $S$ \\
\hline PHL & $W$ & A & A & $\mathrm{D}$ & W & S & A & A & $\mathrm{D}$ & D & & A & $S$ & A & W & $S$ \\
\hline SGP & $\mathrm{D}$ & A & W & $\mathrm{D}$ & $\mathrm{D}$ & $\mathrm{D}$ & A & W & D & $\mathrm{D}$ & $\mathrm{D}$ & & W & $\mathrm{D}$ & W & $\mathrm{D}$ \\
\hline KOR & A & $\mathrm{D}$ & $\mathrm{D}$ & $\mathrm{D}$ & $\mathrm{D}$ & S & A & D & D & S & $\mathrm{D}$ & A & & D & $\mathrm{D}$ & $\mathrm{D}$ \\
\hline THA & $S$ & A & $\mathrm{D}$ & W & $\mathrm{D}$ & S & A & $\mathrm{D}$ & D & W & D & A & S & & W & S \\
\hline VNM & $\mathrm{W}$ & W & W & S & D & S & A & $\mathrm{W}$ & $\mathrm{D}$ & $\mathrm{S}$ & W & W & S & W & & $S$ \\
\hline
\end{tabular}

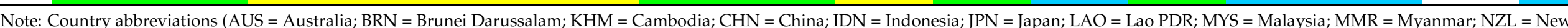

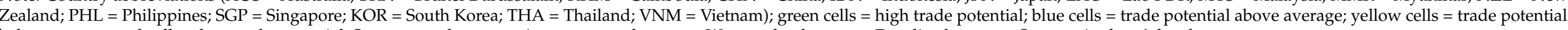
below average; red cells = low trade potential; $\mathrm{S}=$ strong advantage; $\mathrm{A}=$ average advantage; $\mathrm{W}=$ weak advantage; $\mathrm{D}=$ disadvantage. Source: Authors' development. 
Table A14. Degrees of trade potentials and advantages of RCEP countries in trade in prepared and preserved fish and aquatic invertebrates $\left(p_{5}\right)$ in $2000-2019$.

\begin{tabular}{|c|c|c|c|c|c|c|c|c|c|c|c|c|c|c|c|c|}
\hline Countries & AUS & BRN & КHM & CHN & IDN & JPN & LAO & MYS & MMR & NZL & PHL & SGP & KOR & THA & VNM & RCEP \\
\hline AUS & & $\mathrm{A}$ & $\bar{W}$ & $\mathrm{~W}$ & $\mathrm{D}$ & W & $\bar{A}$ & $\mathrm{D}$ & $\mathrm{D}$ & $\mathrm{D}$ & $\mathrm{D}$ & $\mathrm{A}$ & $\mathrm{D}$ & $\mathrm{D}$ & W & $\mathrm{D}$ \\
\hline BRN & W & & $\mathrm{A}$ & W & D & $\mathrm{D}$ & A & W & W & D & D & D & D & D & $\mathrm{D}$ & D \\
\hline KHM & $\mathrm{A}$ & A & & W & D & $\mathrm{D}$ & A & W & A & D & D & D & D & D & W & D \\
\hline $\mathrm{CHN}$ & W & $\mathrm{A}$ & W & & W & S & A & S & D & W & W & A & S & W & W & S \\
\hline IDN & $\mathrm{S}$ & $\mathrm{A}$ & A & $\mathrm{D}$ & & S & A & $\mathrm{D}$ & A & $\mathrm{A}$ & $\mathrm{D}$ & W & A & S & $\mathrm{A}$ & A \\
\hline JPN & $\mathrm{W}$ & $\mathrm{A}$ & $S$ & $\mathrm{D}$ & $\mathrm{D}$ & & S & A & $\mathrm{D}$ & W & D & A & W & D & D & D \\
\hline LAO & D & D & D & D & $\mathrm{D}$ & D & & D & $\mathrm{D}$ & $\mathrm{D}$ & D & D & A & D & W & D \\
\hline MYS & $S$ & $\mathrm{~S}$ & A & $\mathrm{D}$ & $\mathrm{D}$ & A & A & & $\mathrm{D}$ & $\mathrm{W}$ & D & A & A & $\mathrm{D}$ & D & D \\
\hline MMR & S & A & A & S & $\mathrm{D}$ & A & A & A & & $\mathrm{S}$ & A & $\mathrm{W}$ & A & $\mathrm{D}$ & A & A \\
\hline NZL & S & $\mathrm{S}$ & $\mathrm{S}$ & S & $\mathrm{D}$ & A & A & W & D & & D & $\mathrm{S}$ & S & W & $\mathrm{D}$ & S \\
\hline PHL & $S$ & S & A & D & W & A & A & W & $\mathrm{D}$ & S & & A & A & W & W & A \\
\hline SGP & W & A & A & D & W & D & A & W & $\mathrm{W}$ & D & W & & W & D & W & D \\
\hline KOR & A & $\mathrm{A}$ & A & D & $\mathrm{D}$ & S & W & D & D & D & D & W & & D & D & $\mathrm{D}$ \\
\hline THA & S & $S$ & S & $\mathrm{D}$ & $\mathrm{D}$ & S & A & W & W & W & D & S & S & & D & S \\
\hline VNM & S & A & W & W & W & S & A & W & D & S & D & W & S & W & & S \\
\hline
\end{tabular}

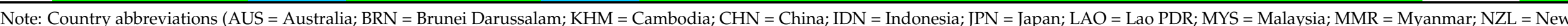

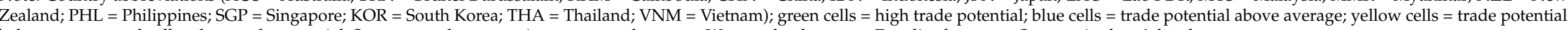
below average; red cells = low trade potential; $\mathrm{S}=$ strong advantage; $\mathrm{A}=$ average advantage; $\mathrm{W}=$ weak advantage; $\mathrm{D}=$ disadvantage. Source: Authors' development. 


\section{References}

1. Pedroza-Gutiérrez, C.; Hernández, J. Social Networks and Supply Chain Management in Fish Trade. Sage Open 2020, 10. [CrossRef]

2. Garcia, S.M.; Rosenberg, A.A. Food Security and Marine Capture Fisheries: Characteristics, Trends, Drivers and Future Perspectives. Philos. Trans. R. Soc. B Biol. Sci. 2010, 365, 2869-2880. [CrossRef] [PubMed]

3. Kittinger, J.; Teneva, L.; Koike, H.; Stamoulis, K.; Kittinger, D.; Oleson, K.; Conkin, E.; Gomes, M.; Wilcox, B.; Friedlander, A. From Reef to Table: Social and Ecological Factors Affecting Coral Reef Fisheries, Artisanal Seafood Supply Chains, and Seafood Security. PLoS ONE 2015, 10, e0123856. [CrossRef] [PubMed]

4. Allison, E.; Perry, A.; Badjeck, M.C.; Neil Adger, W.; Brown, K.; Conway, D.; Halls, A.; Pilling, G.; Reynolds, J.; Andrew, N.; et al. Vulnerability of National Economies to the Impacts of Climate Change on Fisheries. Fish Fish. 2009, 10, 173-196. [CrossRef]

5. Yegorov, Y. Sustainable Development of Oil Production in the Arctic Shelf and Evolution of Fish Stock. In Handbook of Research on International Collaboration, Economic Development, and Sustainability in the Arctic; Erokhin, V., Gao, T., Zhang, X., Eds.; IGI Global: Hershey, PA, USA, 2019; pp. 451-469. [CrossRef]

6. Fox, M.; Mitchell, M.; Dean, M.; Elliott, C.; Campbell, K. The Seafood Supply Chain from a Fraudulent Perspective. Food Secur. 2018, 10, 939-963. [CrossRef]

7. Shehata, H.R.; Bourque, D.; Steinke, D.; Chen, S.; Hanner, R. Survey of Mislabelling across Finfish Supply Chain Reveals Mislabelling both outside and within Canada. Food Res. Int. 2019, 121, 723-729. [CrossRef]

8. Kumar, R.; Kumar, R.R.; Stauvermann, P.J.; Arora, P. Effect of Fisheries Subsidies Negotiations on Fish Production and Interest Rate. J. Risk Financ. Manag. 2020, 13, 297. [CrossRef]

9. Erokhin, V.; Gao, T. Handbook of Research on Globalized Agricultural Trade and New Challenges for Food Security; IGI Global: Hershey, PA, USA, 2020. [CrossRef]

10. Food and Agriculture Organization. How Is COVID-19 Affecting the Fisheries and Aquaculture Food Systems; Food and Agriculture Organization of the United Nations: Rome, Italy, 2020.

11. Bailey, M.; Bush, S.R.; Miller, A.; Kochen, M. The Role of Traceability in Transforming Seafood Governance in the Global South. Curr. Opin. Environ. Sustain. 2016, 18, 25-32. [CrossRef]

12. Food and Agriculture Organization. The State of World Fisheries and Aquaculture 2020; Food and Agriculture Organization of the United Nations: Rome, Italy, 2020.

13. Kanaga, V.; Radhakrishnan, K.; Kumar, S.R.; Karthy, A.; Vijayarahavan, V. Influencing Variable Effect on Fish Trade a View with Liberalization and WTO Regime. Indian J. Econ. Dev. 2017, 13, 693-700. [CrossRef]

14. Shamsuzzaman, M.M.; Mozumder, M.M.H.; Mitu, S.J.; Ahamad, A.F.; Bhyuian, S.M. The Economic Contribution of Fish and Fish Trade in Bangladesh. Aquac. Fish. 2020, 5, 174-181. [CrossRef]

15. Devadason, E.S.; Mubarik, S. ASEAN and the EU: An Assessment of Interregional Trade Potentials. Int. Econ. Econ. Policy 2020, 17, 705-726. [CrossRef]

16. Nielsen, M. Modelling Fish Trade Liberalisation: Does Fish Trade Liberalisation Result in Welfare Gains or Losses? Mar. Policy 2009, 33, 1-7. [CrossRef]

17. Ruland, J. ASEAN and the European Union: A Bumpy Inter-Regional Relationship; University of Bonn: Bonn, Germany, 2001.

18. Katada, S.N.; Solis, M. Cross Regional Trade Agreements: Understanding Permeated Regionalism in East Asia; Springer: Heidelberg, Germany, 2008. [CrossRef]

19. Mansfield, E.D.; Pevehouse, J.C.W. The Expansion of Preferential Trading Arrangements. Int. Stud. Q. 2013, 57, 592-604. [CrossRef]

20. Bano, S.; Takahashi, Y.; Scrimgeour, F. ASEAN-New Zealand Trade Relations and Trade Potential: Evidence and Analysis. J. Econ. Integr. 2013, 28, 144-182. [CrossRef]

21. Regional Comprehensive Economic Partnership. About Regional Comprehensive Economic Partnership (RCEP). Available online: https:/ / rcepsec.org/about/ (accessed on 31 January 2021).

22. Erokhin, V. Regional Comprehensive Economic Partnership: A Review of Major Parameters of the Trade Alliance. Mark. Logist. 2020, 32, 23-34.

23. Regional Comprehensive Economic Partnership. ASEAN Hits Historic Milestone with Signing of RCEP. Available online: https: / rcepsec.org/2020/11/26/asean-hits-historic-milestone-with-signing-of-rcep/ (accessed on 31 January 2021).

24. Chan, C.Y.; Tran, N.; Dao, C.D.; Sulser, T.B.; Phillips, M.J.; Batka, M.; Wiebe, K.; Preston, N. Fish to 2050 in the ASEAN Region; WorldFish: Penang, Malaysia; International Food Policy Research Institute: Washington, DC, USA, 2017.

25. Barlow, C.; Baran, E.; Halls, A.S.; Kshatriya, M. How Much of the Mekong Fish Catch Is at Risk from Mainstream Dam Development? Catch Cult. 2008, 14, 16-21.

26. Dugan, P.J.; Barlow, C.; Agostinho, A.A.; Baran, E.; Cada, G.F.; Chen, D.; Cowx, I.G.; Ferguson, J.W.; Jutagate, T.; Mallen-Cooper, M.; et al. Fish Migration, Dams, and Loss of Ecosystem Services in the Mekong Basin. Ambio 2010, 39, 344-348. [CrossRef] [PubMed]

27. Ziv, G.; Baran, E.; Nam, S.; Rodriguez-Iturbe, I.; Levin, S.A. Trading-off Fish Biodiversity, Food Security, and Hydropower in the Mekong River Basin. PNAS 2012, 109, 5609-5614. [CrossRef] [PubMed] 
28. Béné, C. Small-Scale Fisheries: Assessing Their Contribution to Rural Livelihoods in Developing Countries; Food and Agriculture Organization of the United Nations: Rome, Italy, 2006.

29. Teh, L.; Pauly, D. Who Brings in the Fish? The Relative Contribution of Small-Scale and Industrial Fisheries to Food Security in Southeast Asia. Front. Mar. Sci. 2018, 5, 44. [CrossRef]

30. Pomeroy, R.; Nguyen, K.; Thong, H. Small-Scale Marine Fisheries Policy in Vietnam. Mar. Policy 2009, 33, 419-428. [CrossRef]

31. Ali, M.; Yongtong, M.; Oad, S.; Shah, S.B.H.; Kalhoro, M.A.; Kalhoro, M.T.; Lahbar, G.M. A Comparative Analysis on Expansion of Pakistan Fisheries Trade: World and China. Indian J. Geo Mar. Sci. 2020, 49, 1643-1650.

32. Needham, S.; Funge-Smith, S. The Consumption of Fish and Fish Products in the Asia-Pacific Region Based on Household Surveys; FAO Regional Office for Asia and the Pacific: Bangkok, Thailand, 2014.

33. Tveteras, S.; Asche, F. International Fish Trade and Exchange Rates: An Application to the Trade with Salmon and Fishmeal. Appl. Econ. 2008, 40, 1745-1755. [CrossRef]

34. Erokhin, V. Establishing Food Security and Alternatives to International Trade in Emerging Economies; IGI Global: Hershey, PA, USA, 2018. [CrossRef]

35. Einarsson, Á.; Óladóttir, Á.D. Fisheries and Aquaculture: The Food Security of the Future; Elsevier: Amsterdam, The Netherlands, 2020.

36. United Nations. Sustainable Development Goal 2: End Hunger, Achieve Food Security and Improved Nutrition and Promote Sustainable Agriculture. Available online: https:/ /sdgs.un.org/goals/goal2 (accessed on 31 January 2021).

37. Honniball, A. Unilateral Trade Measures and the Importance of Defining IUU Fishing: Lessons from the 2019 USA "Concerns" with China as a Fishing Flag State. J. Territ. Marit. Stud. 2020, 7, 7-26. [CrossRef]

38. United Nations. Sustainable Development Goal 14: Conserve and Sustainably Use the Oceans, Seas and Marine Resources for Sustainable Development. Available online: https://sdgs.un.org/goals/goal14 (accessed on 31 January 2021).

39. Tall, A. Identifying Policy Synergies on Aid for Trade, Fisheries, and Food Security; International Centre for Trade and Sustainable Development: Geneva, Switzerland, 2015.

40. Latif, B.; Noor, S.M.; Sumardi, J.; Irwansyah, I. Sustainable Development and Sea Protection: Trade on Fish and Fishery Product. IOP Conf. Ser. Earth Environ. Sci. 2019, 343, 012069. [CrossRef]

41. Bellmann, C.; Tipping, A.; Sumaila, R.U. Global Trade in Fish and Fishery Products: An Overview. Mar. Policy 2016, 69, 181-188. [CrossRef]

42. Southeast Asian Fisheries Development Center. Fishery Statistical Bulletin of Southeast Asia 2018; Southeast Asian Fisheries Development Center: Bangkok, Thailand, 2020.

43. Delgado, C.; Wada, N.; Rosegrant, M.; Meijer, S.; Ahmed, M. Fish to 2020: Supply and Demand in Changing Global Markets; International Food Policy Research Institute: Washington, DC, USA, 2003.

44. Mossler, M. State of World Fisheries and Aquaculture 2020. Available online: https:/ / sustainablefisheries-uw.org/fao-state-ofworld-fisheries-2020/ (accessed on 30 January 2021).

45. United Nations Conference on Trade and Development. Data Center. Available online: https://unctadstat.unctad.org/EN/ (accessed on 24 January 2021).

46. Campling, L. Tariff Escalation and Preferences in International Fish Production and Trade; International Centre for Trade and Sustainable Development: Geneva, Switzerland, 2015.

47. Park, D.; Park, I.; Estrada, G.E.B. Is the ASEAN-Korea Free Trade Area (AKFTA) an Optimal Free Trade Area; Asian Development Bank: Manila, Philippines, 2008.

48. Prabhakar, A.C.; Kaur, G.; Erokhin, V. Regional Trade and Development Strategies in the Era of Globalization; IGI Global: Hershey, PA, USA, 2020. [CrossRef]

49. Yue, C.S. The ASEAN Free Trade Area. Pac. Rev. 1998, 11, 213-232. [CrossRef]

50. Erokhin, V. Global Perspectives on Trade Integration and Economies in Transition; IGI Global: Hershey, PA, USA, 2016. [CrossRef]

51. Menon, J. Transitional Economies in Free Trade Areas: Lao PDR in the ASEAN Free Trade Area. J. Asia Pac. Econ. 1999, 4, 340-364. [CrossRef]

52. Mukherji, I.N. The Bangkok Agreement: A Negative List Approach to Trade Liberalization in Asia and the Pacific. In Proceedings of the 19th Session of the Standing Committee of the Bangkok Agreement, Bangkok, Thailand, 27-28 October 2003; The Economic and Social Commission for Asia and the Pacific: Bangkok, Thailand, 2003.

53. Mukherji, I.N. Asia-Pacific Trade Agreement: Implications of Exchange of Trade Preferences for Member and Prospective Member Countries. Asia Pac. Trade Invest. Rev. 2007, 3, 87-144.

54. Bano, S. ASEAN-New Zealand Trade Relations and Trade Potential; University of Waikato: Hamilton, New Zealand, 2010.

55. Kaur, N.; Sarin, V. Trade Potential of India and ASEAN Nations. Mukt Shabd J. 2020, 9, 307-314.

56. Qayyum, U.; Nigar, N. An Empirical Analysis of Pakistan's Agriculture Trade with China: Complementarity or Competition; Pakistan Institute of Development Economics: Islamabad, Pakistan, 2020.

57. Devadason, E.S.; Chandran Govindaraju, V.G.R. Unlocking the Trade Potential in China-ASEAN Relations: The China-Vietnam Context. Southeast Asian Econ. 2020, 36, 380-399. [CrossRef]

58. Abedini, J.; Péridy, N. The Emergence of Iran in the World Car Industry: An Estimation of its Export Potential. World Econ. 2009, 32, 790-818. [CrossRef]

59. Sharma, S.C.; Chua, S.Y. ASEAN: Economic Integration and Intra-Regional Trade. Appl. Econ. Lett. 2000, 7, 165-169. [CrossRef]

60. Roberts, B. A Gravity Study of the Proposed China-ASEAN Free Trade Area. Int. Trade J. 2004, 18, 335-353. [CrossRef] 
61. Ibitoye, O. Assessment of the Potential Level of Food Trade in the ECOWAS Region. J. Econ. Trade 2020, 5, 35-51.

62. Helmers, C.; Pasteels, J.-M. Assessing Bilateral Trade Potential at the Commodity Level: An Operational Approach; International Trade Center: Geneva, Switzerland, 2006.

63. Li, W.; Li, C. The Empirical Study on Potential of Bilateral Trade between China and Kenya in the Context "The Belt and Road". Theor. Econ. Lett. 2020, 10, 119-130. [CrossRef]

64. Kapuya, T.; Chinembiri, E.; Kalaba, M. Identifying Strategic Markets for South Africa's Citrus Exports. Agrekon 2014, 53, 124-158. [CrossRef]

65. Kaur, S.; Khorana, S.; Kaur, M. Is There Any Potential in Service Trade of South Asia? Foreign Trade Rev. 2020, 55, 402-417. [CrossRef]

66. Sanidas, E.; Shin, Y. Comparison of Revealed Comparative Advantage Indices with Application to Trade Tendencies of East Asian Countries; Seoul National University: Seoul, Korea, 2010.

67. Svatos, M.; Smutka, L.; Miffek, O. Competitiveness of Agrarian Trade of EU-15 Countries in Comparison with New EU Member States. Agric. Econ. Zemed. Ekon. 2010, 56, 569-582. [CrossRef]

68. Balassa, B. Trade Liberalization and Revealed Comparative Advantage. Manch. Sch. Econ. Soc. Sci. 1965, 33, 99-123. [CrossRef]

69. Wardani, M.A.; Mulatsih, S.; Rindayati, W. Competitiveness and Factors Affecting Indonesian Food Industry's Export to Regional Comprehensive Economic Partnership. Etikonomi 2018, 17, 185-198. [CrossRef]

70. Hoang, V.V. Investigating the Agricultural Competitiveness of ASEAN Countries. J. Econ. Stud. 2020, 47, 307-332. [CrossRef]

71. Nguyen, D.P.; Vo, X.V. Determinants of Bilateral Trade: Evidence from ASEAN+3. Asian Pac. Econ. Lit. 2017, 31, 115-122. [CrossRef]

72. Luo, X.; Han, Y.; Li, Z. Comparing Quantitative Analysis on Revealed Comparative Advantages of Aquatic Products Trade of China and ASEAN Based on 21st Century Maritime Silk Road. IOP Conf. Ser. Earth Environ. Sci. 2017, 94, 012032. [CrossRef]

73. Tampubolon, J. RCA Analysis with Selected Products to Enhance Prediction Power of Competitiveness. J. Ekon. Dan Studi Pembang. 2019, 11, 143-157. [CrossRef]

74. Lee, S.M. Revealed Comparative Advantage of Selected ASEAN Countries' Crustacean Export to Japan. IOP Conf. Ser. Earth Environ. Sci. 2020, 414, 012010. [CrossRef]

75. Kwak, S.Y.; Kim, M.J. A Study on the Analysis of International Trade Competitiveness between Korea and Vietnam. Int. Commer. Inf. Rev. 2018, 20, 49-74. [CrossRef]

76. Kuldilok, K.S.; Dawson, P.J.; Lingard, J. The Export Competitiveness of the Tuna Industry in Thailand. Br. Food J. 2013, 115, 328-341. [CrossRef]

77. Sutawi, S.; Hendraningsih, L.; Wahyudi, A. Competitiveness of Indonesian Beef Trading in ASEAN. J. Indones. Trop. Anim. Agric. 2019, 44, 213-219. [CrossRef]

78. Jain, M. Review of ASEAN-India Free Trade Agreement Using Revealed Comparative Advantage Index. Int. J. Bus. Econ. 2020, 5, $56-65$.

79. Laosutsan, P.; Shivakoti, G.; Soni, P. Comparative Advantage and Export Potential of Thai Vegetable Products Following the Integration into the ASEAN Economic Community. Int. Food Agribus. Manag. Rev. 2017, 20, 575-590. [CrossRef]

80. Erokhin, V.; Li, D.; Du, P. Sustainability-Related Implications of Competitive Advantages in Agricultural Value Chains: Evidence from Central Asia-China Trade and Investment. Sustainability 2020, 12, 1117. [CrossRef]

81. Leromain, E.; Orefice, G. New Revealed Comparative Advantage Index: Dataset and Empirical Distribution. Int. Econ. 2014, 139, 48-70. [CrossRef]

82. Ballance, R.H.; Forstner, H.; Murray, T. Consistency Tests of Alternative Measures of Comparative Advantage. Rev. Econ. Stat. 1987, 69, 157-161. [CrossRef]

83. Coniglio, N.; Vurchio, D.; Cantore, N.; Clara, M. On the Evolution of Comparative Advantage: Path-Dependent Versus Path-Defying Changes; University of Bari Aldo Moro: Bari, Italy, 2018.

84. Gnidchenko, A.; Salnikov, V. Net Comparative Advantage Index: Overcoming the Drawbacks of the Existing Indices; National Research University Higher School of Economics: Moscow, Russia, 2015.

85. Yeats, A.J. On the Appropriate Interpretation of the Revealed Comparative Advantage Index: Implications of a Methodology Based on Industry Sector Analysis. Weltwirtschaftliches Arch. 1985, 121, 61-73. [CrossRef]

86. Hoen, A.R.; Oosterhaven, J. On the Measure of Comparative Advantage. Ann. Reg. Sci. 2006, 40, 677-691. [CrossRef]

87. Khai, N.X.; Ismail, M.M.; Sidique, S.F. Consistency Tests of Comparative Advantage Measures: An Empirical Evidence from the Malaysian and Selected Asian Shrimp Products. Int. Food Res. J. 2016, 23, 2752-2758.

88. Rusali, M.; Gavrilescu, C. Competitive Advantages and Disadvantages in Romania's Agri-Food Trade-Trends and Challenges. In Proceedings of the 12th Congress of the European Association of Agricultural Economists, Ghent, Belgium, 26-29 August 2008; European Association of Agricultural Economists: Ghent, Belgium, 2008.

89. Drabik, D.; Bartova, L. An Assessment of the Slovak Agri-Food Trade Specialisation Pattern. Ekonomický Časopis 2009, 57, 3-20.

90. Carraresi, L.; Banterle, A. Agri-Food Competitive Performance in EU Countries: A Fifteen-Year Retrospective. Int. Food Agribus. Manag. Rev. 2015, 18, 37-62. [CrossRef]

91. Campos, F.J.A.; Chavez, J.C.L.N.; Ortega, O.V.D. Competitiveness and Productivity of Mexican Agricultural Sector in APEC, 1980-2015. Portes Rev. Mex. De Estud. Sobre La Cuenca Del Pac. 2018, 12, 7-30.

92. Bashimov, G. Turkey's Export Performance of Tomato and Competitiveness. Alinteri J. Agric. Sci. 2016, 31, 1-8. [CrossRef] 
93. Zaghini, A. Trade Advantages and Specialisation Dynamics in Acceding Countries; European Central Bank: Frankfurt am Main, Germany, 2003.

94. Ferto, I.; Hubbard, L.J. Revealed Comparative Advantage and Competitiveness in Hungarian Agrifood Sector. World Econ. 2003, 26, 247-259. [CrossRef]

95. Erokhin, V.; Gao, T. Competitive Advantages of China's Agricultural Exports in the Outward-Looking Belt and Road Initiative. In China's Belt and Road Initiative: Changing the Rules of Globalization; Zhang, W., Alon, I., Lattemann, C., Eds.; Palgrave Macmillan: London, UK, 2018; pp. 265-285. [CrossRef]

96. Karimi, M.S.; Malekshahian, M. Ricardian Comparative Advantage: Impact of Specialization on the Exportation of Products in ASEAN Countries. Ann. Econ. Financ. 2018, 19, 473-522.

97. Oberoi, S. Dynamics of Trade Specialization and Trade Performance of ASEAN-India Free Trade Agreement. J. Int. Commer. Econ. Policy 2019, 10, 1950003. [CrossRef]

98. Smutka, L.; Pokorna, I.; Pulkrabek, J. Regional Analysis of Competitiveness of Raw and White Sugar. Listy Cukrov. A Reparske 2012, 128, 141-145.

99. Maitah, M.; Kuzmenko, E.; Smutka, L. Real Effective Exchange Rate of Rouble and Competitiveness of Russian Agrarian Producers. Economies 2016, 4, 12. [CrossRef]

100. Benesova, I.; Maitah, M.; Smutka, L.; Tomsik, K.; Ishchukova, N. Perspectives of the Russian Agricultural Exports in Terms of Comparative Advantage. Agric. Econ. Zemed. Ekon. 2017, 63, 318-330. [CrossRef]

101. Benesova, I.; Smutka, L.; Laputkova, A. Specifics of the Mutual Trade of the Post-Soviet Countries. Entrep. Bus. Econ. Rev. 2019, 7, 29-43. [CrossRef]

102. Szczepaniak, I. Changes in Comparative Advantages of the Polish Food Sector in World Trade. Equilibrium. Q. J. Econ. Econ. Policy 2019, 14, 463-480. [CrossRef]

103. Allegra, V.; Zarba, C.; La Via, G.; Zarba, A.S. Why the New Orange Juice Consumption Model Favors Global Trade and Growth in Orange Production. Br. Food J. 2019, 121, 1954-1968. [CrossRef]

104. Ignjatijevic, S.; Ciric, M.; Caric, M. International Trade Structure of Countries from the Danube Region: Comparative Advantage Analysis of Export. Ekon. Cas. 2013, 61, 251-269.

105. Smutka, L.; Maitah, M.; Svatos, M. Changes in the Czech Agrarian Foreign Trade Competitiveness-Different Groups of Partners' Specifics. Agric. Econ. Zemed. Ekon. 2018, 64, 399-411. [CrossRef]

106. Ortikov, A.; Vacek, T. Comparative Advantage: Products Mapping of Uzbekistan's Agricultural Exports. In Proceedings of the 27th International Scientific Conference "Agrarian Perspectives XXVII—Food Safety-Food Security”, Prague, Czech Republic, 19-20 September 2018; Czech University of Life Sciences Prague: Prague, Czech Republic, 2018.

107. Ortikov, A. Changes in the Character and Competitiveness of Uzbekistan's Agrarian Foreign Trade. In Proceedings of the 26th International Scientific Conference "Agrarian Perspectives XXVII-Competitiveness of European Agriculture and Food Sectors", Prague, Czech Republic, 13-15 September 2017; Czech University of Life Sciences Prague: Prague, Czech Republic, 2017.

108. Verter, N.; Zdrahal, I.; Becvarova, V.; Grega, L. "Products Mapping” and Trade in Agri-Food Products between Nigeria and the EU28. Agric. Econ. Zemed. Ekon. 2020, 66, 34-45. [CrossRef]

109. Rundassa, M.W.; Azene, D.K.; Berhan, E. Comparative Advantage of Ethiopian Textile and Apparel Industry. Res. J. Text. Appar. 2019, 23, 244-256. [CrossRef]

110. Alessandrini, M.; Fattouh, B.; Scaramozzino, P. The Changing Pattern of Foreign Trade Specialization in Indian Manufacturing. Oxf. Rev. Econ. Policy 2007, 23, 270-291. [CrossRef]

111. Ishchukova, N. The Position of the Russian Federation in the International Market of Agricultural and Foodstuff Products; Czech University of Life Sciences Prague: Prague, Czech Republic, 2013.

112. Dunmore, J.C. Competitiveness and Comparative Advantage of U.S. Agriculture. Increasing Underst. Public Probl. Policies 1986, 21-34. [CrossRef]

113. Siggel, E. International Competitiveness and Comparative Advantage: A Survey and a Proposal for Measurement. J. Ind. Compet. Trade 2006, 6, 137-159. [CrossRef]

114. Costinot, A.; Donaldson, D.; Komunjer, I. What Goods Do Countries Trade? A Quantitative Exploration of Ricardo's Ideas. Rev. Econ. Stud. 2012, 79, 581-608. [CrossRef]

115. Hinloopen, J.; Van Marrewijk, C. On the Empirical Distribution of the Balassa index. Weltwirtschaftliches Arch. 2001, 137, 1-35. [CrossRef]

116. Kim, H.Y. The Economic Effect and Implications of a FTA on Busan Fisheries Industry. J. Int. Trade Ind. Stud. 2008, 13, 73-103.

117. Shah, S.; Kamal, M.; Yu, D. Did China-Pakistan Free Trade Agreement Promote Trade and Development in Pakistan? Int. J. Financ. Econ. 2020, 27. [CrossRef]

118. Lin, X.; Kim, K.S. A Study on the Determinant of Korean Fisheries Export to ASEAN. J. Fish. Bus. Adm. 2016, 47, 15-32. [CrossRef]

119. Choi, S.; Choi, H. Economic Effects of Korea-Singapore Free Trade Agreement on the Fisheries Sector. J. Fish. Bus. Adm. 2004, 35, 71-90.

120. Abelson, P. The Trade Deficit. Science 1987, 236, 653. [CrossRef] [PubMed]

121. Feldstein, M. Correcting the Trade Deficit. Foreign Aff. 1987, 65, 795-806. [CrossRef]

122. Mishra, R.K.; Karthik, M. Understanding the US-China Trade War: Causes, Consequences and Economic Impact. Glob. Trade Cust. J. 2020, 15, 580-591. 
123. Belke, A.; Domnick, C. Trade and Capital Flows: Substitutes or Complements? An Empirical Investigation. Rev. Int. Econ. 2021. [CrossRef]

124. Krueger, A. International Trade: What Everyone Needs to Know; Oxford University Press: Oxford, UK, 2020. [CrossRef]

125. Andreosso-O'Callaghan, B.; Nicolas, F. Are the Economies of ASEAN and the EU Complementary? Asean Econ. Bull. 2007, 24, 205-224. [CrossRef]

126. Kurien, J. Responsible Fish Trade and Food Security; Food and Agriculture Organization of the United Nations: Rome, Italy, 2005.

127. Teh, L.; Zeller, D.; Zylich, K.; Nguyen, G.; Harper, S. Reconstructing Vietnam's Marine Fisheries Catch, 1950-2010; University of British Columbia: Vancouver, BC, Canada, 2014.

128. Teh, L.; Zeller, D.; Pauly, D. Preliminary Reconstruction of Thailand's Fisheries Catches: 1950-2010; University of British Columbia: Vancouver, BC, Canada, 2015.

129. Garcia Garcia, S.; Barclay, K.; Nicholls, R. Can Anti-Illegal, Unreported, and Unregulated (IUU) Fishing Trade Measures Spread Internationally? Case Study of Australia. Ocean Coast. Manag. 2021, 202, 105494. [CrossRef]

130. Steven, A.H.; Mobsby, D.; Curtotti, R. Australian Fisheries and Aquaculture Statistics 2018; Australian Bureau of Agricultural and Resource Economics and Sciences: Canberra, Australia, 2020.

131. Fabinyi, M. The Chinese Seafood Market: Opportunities and Challenges for Australian Exporters; University of Technology Sydney: Sydney, Australia, 2018.

132. Wardono, B.; Muhartono, R.; Apriliani, T.; Hikmah Zulham, A. Traditional Fisheries Supply Chain Management in the Border Area: The Natuna Islands, Indonesia. E3s Web Conf. 2020, 147, 02009. [CrossRef]

133. Jaya, S. International Fish Trade: A Case Study of Indonesia. In Proceedings of the SEAFDEC Fish Trade Meeting, Bangkok, Thailand, 7-8 June 2018; Southeast Asian Fisheries Development Center: Bangkok, Thailand, 2018.

134. Tran, N.; Wilson, N.; Hite, D. Choosing the Best Model in the Presence of Zero Trade: A Fish Product Analysis. In Nontariff Measures with Market Imperfections: Trade and Welfare Implications; Beghin, J.C., Ed.; Emerald Group Publishing Limited: Bingley, UK, 2013; pp. 127-149. [CrossRef]

135. Henson, S.; Jaffee, S. Understanding Developing Country Strategic Responses to the Enhancement of Food Safety Standards. World Econ. 2008, 31, 548-568. [CrossRef]

136. Surahman, Z.M.; Hanningtyas, I.; Aristi, D.; Cahyaningrum, F.; Laelasari, E. Factors Related to the Presence of Formaldehyde in the Salted Fish Trade in Ciputat, Indonesia. Malays. J. Med. Health Sci. 2019, 15, 89-94.

137. White, E.; Froehlich, H.; Gephart, J.; Cottrell, R.; Branch, T.; Bejarano, R.A.; Baum, J. Early Effects of COVID-19 on US Fisheries and Seafood Consumption. Fish Fish. 2020, 22, 232-239. [CrossRef] [PubMed]

138. Bennett, N.; Finkbeiner, E.; Ban, N.; Belhabib, D.; Jupiter, S.; Kittinger, J.; Mangubhai, S.; Scholtens, J.; Gill, D.; Christie, P. The COVID-19 Pandemic, Small-Scale Fisheries and Coastal Fishing Communities. Coast. Manag. 2020, 48, 336-347. [CrossRef]

139. Supartini, A.; Oishi, T.; Yagi, N. Changes in Fish Consumption Desire and Its Factors: A Comparison between the United Kingdom and Singapore. Foods 2018, 7, 97. [CrossRef] [PubMed]

140. De Koninck, R. Farmers of a City State: The Chinese Smallholders of Singapore; Universite Laval: Laval, QC, Canada, 1973.

141. Wong, C.Y.; Lim, G. A Typology of Agricultural Production Systems: Capability Building Trajectories of Three Asian Economies. Asia Pac. Viewp. 2020, 61,37-53. [CrossRef]

142. Brander, J.; Taylor, M. Open Access Renewable Resources Trade and Trade Policy in a Two-Country Model. J. Int. Econ. 1998, 44, 181-209. [CrossRef]

143. Emami, A.; Johnson, R. Unilateral Resource Management in a 2-Country General Equilibrium Model. Am. J. Agric. Econ. 2000, 82, 161-172. [CrossRef]

144. Kathuria, S. Why Trade Liberalization Benefits Smaller Countries More. Available online: https://www.weforum.org/agenda/ 2015/02/why-trade-liberalisation-benefits-smaller-countries-more/ (accessed on 3 February 2021).

145. Sneddon, C.; Fox, C. Inland Capture Fisheries and Large River Systems: A Political Economy of Mekong Fisheries. J. Agrar. Chang. 2012, 12, 279-299. [CrossRef]

146. Chap, S.; Meng, K.; Tep, C.; Joffre, O. Crab Fisheries in Cambodia and the Development of Crab Banks; The Learning Institute and the WorldFish Center: Phnom Penh, Cambodia, 2012.

147. Srean, P.; Sinh, K.; Prak, S.; Bin, C. Potency of Aquatic Local Resources in Cambodia. In Proceedings of the International Seminar on Promoting Local Resources for Food and Health, Bengkulu, Indonesia, 12-13 October 2015; ISEPROLOCAL: Bengkulu, Indonesia, 2015.

148. Martin, S.; Lorenzen, K.; Bunnefeld, N. Fishing Farmers: Fishing, Livelihood Diversification and Poverty in Rural Laos. Hum. Ecol. 2013, 41, 737-747. [CrossRef]

149. Hartje, R.; Buhler, D.; Grote, U. Eat Your Fish and Sell It, Too-Livelihood Choices of Small-Scale Fishers in Rural Cambodia. Ecol. Econ. 2018, 154, 88-98. [CrossRef]

150. Joffre, O.M.; Castine, S.A.; Phillips, M.J.; Sellamuttu, S.S.; Chandrabalan, D.; Cohen, P. Increasing Productivity and Improving Livelihoods in Aquatic Agricultural Systems: A Review of Interventions. Food Secur. 2017, 9, 39-60. [CrossRef]

151. Patricio, H.; Zipper, S.; Peterson, M.; Ainsley, S.; Loury, E.; Ounboundisane, S.; Demko, D. Fish Catch and Community Composition in a Data-Poor Mekong River Subcatchment Characterised through Participatory Surveys of Harvest from an Artisanal Fishery. Mar. Freshw. Res. 2018, 70, 153-168. [CrossRef] 
152. Horlings, J.; Marschke, M. Fishing, Farming and Factories: Adaptive Development in Coastal Cambodia. Clim. Dev. 2019, 12, 521-531. [CrossRef]

153. Ebil, S.; Sheppard, C.; Wahab, R.; Price, A.; Bull, J. Changes in Community Structure of Finfish Catches in Brunei Darussalam between 2000 and 2009. Ocean Coast. Manag. 2013, 76, 45-51. [CrossRef]

154. Teh, L.; Shon, D.; Zylich, K.; Zeller, D. Reconstructing Cambodia's Marine Fisheries Catch, 1950-2010; University of British Columbia: Vancouver, BC, Canada, 2014.

155. Kim, S. A Study on the Effects of Regional Trade Agreements on the Trade of Fishery Products in Korea. J. Korea Res. Assoc. Int. Commer. 2019, 19, 79-97. [CrossRef]

156. Anderson, J.; Van Wincoop, E. Gravity with Gravitas: A Solution to the Border Puzzle. Am. Econ. Rev. 2003, 93, 170-192. [CrossRef]

157. Péridy, N. The New Trade Policy between the USA and MENA Countries: A Quantitative Assessment. World Econ. 2005, 28, 491-518. [CrossRef]

158. Kim, B. Pricing Behavior for Sustainably Farmed Fish in International Trade: The Case of Norwegian Atlantic Salmon (Salmo Salar). Sustainability 2018, 10, 4814. [CrossRef]

159. Fishe, R.; Janzen, J.; Smith, A. Hedging and Speculative Trading in Agricultural Futures Markets. Am. J. Agric. Econ. 2014, 96, 542-556. [CrossRef]

160. Harrigan, J. Scale Economies and the Volume of Trade. Rev. Econ. Stat. 1994, 76, 321-328. [CrossRef]

161. Mohammadi, H.; Saghaian, S.; Aminizadeh, M.; Aghasafari, H. Food Safety Standards and Their Effects on Iran's Fish Exports. Iran. J. Fish. Sci. 2020, 19, 3075-3085. [CrossRef]

162. Santeramo, F.G.; Lamonaca, E. The Effects of Non-Tariff Measures on Agri-Food Trade: A Review and Meta-Analysis of Empirical Evidence. J. Agric. Econ. 2019, 70, 595-617. [CrossRef]

163. Hammarlund, C.; Jonsson, P.; Valentinsson, D.; Waldo, S. Economic and Environmental Effects of Replacing Bottom Trawling with Fishing with Creels. Aust. J. Agric. Resour. Econ. 2021, 65, 94-118. [CrossRef]

164. Lindsay, A.; Sanchirio, J.; Gilliland, T.; Ambo-Rappe, R.; Taylor, J.E.; Krueck, N.; Mumby, P. Evaluating Sustainable Development Policies in Rural Coastal Economies. Proc. Natl. Acad. Sci. USA 2020, 117, 33170-33176. [CrossRef] [PubMed] 